James Madison University JMU Scholarly Commons

Senior Honors Projects, 2010-current

Honors College

Spring 2019

\title{
Environmental monitoring using a drone-enabled wireless sensor network
}

\author{
Gina Valentino \\ Laura Yates \\ James Madison University \\ Brooke Potter \\ James Madison University
}

Follow this and additional works at: https://commons.lib.jmu.edu/honors201019

Part of the Digital Communications and Networking Commons, and the Environmental Monitoring Commons

\section{Recommended Citation}

Valentino, Gina; Yates, Laura; and Potter, Brooke, "Environmental monitoring using a drone-enabled wireless sensor network" (2019). Senior Honors Projects, 2010-current. 723.

https://commons.lib.jmu.edu/honors201019/723

This Thesis is brought to you for free and open access by the Honors College at JMU Scholarly Commons. It has been accepted for inclusion in Senior Honors Projects, 2010-current by an authorized administrator of JMU Scholarly Commons. For more information, please contact dc_admin@jmu.edu. 


\section{JAMES MADison University \\ Integrated Science \& Technology (ISAT) \\ Senior Capstone}

\section{Environmental Monitoring Using a Drone-Enabled Wireless Sensor Network}

\section{Author(s):}

Brooke PotTer

Student ID: 110846268

Gina Valentino

Student ID: 109607548

Laura Yates

Student ID: 109802055

April 3, 2019

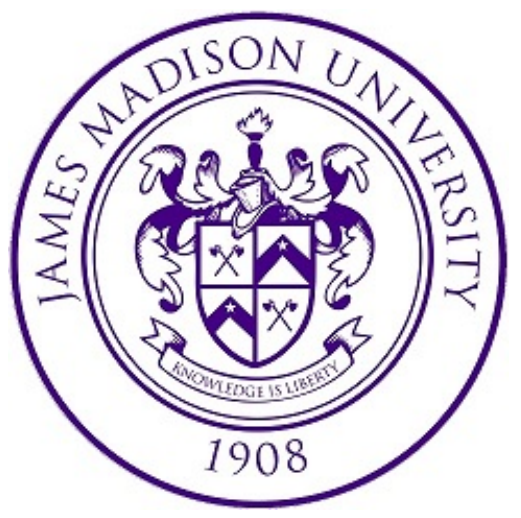

Brooke Potter

Gina Valentino

Laura Yates

Signature

Signature

Signature

Date

Date

Date

Thomas R. Benzing, Ph.D.

Ahmand A. Salman, Ph.D. 


\section{Contents}

$1 \quad$ Abstract . . . . . . . . . . . . . . . 8

2 Introduction to Environmental Monitoring and Regional Context 9

2.1 DuPont Mercury Contamination of the South River in

Waynesboro, Virginia . . . . . . . . . . . 9

2.2 Boones Run . . . . . . . . . . . . . . . . . . 11

2.3 Public Opinion Regarding the Impairments of the South

River ...................... 13

2.4 Industrial Norms of Non-Regulated Disposal of Material

in the South River . . . . . . . . . . . . . . . . . . . . 14

3 Water Quality and Data Collection . . . . . . . . . . . 15

$3.1 \quad$ Background Information . . . . . . . . . . . . . 15

3.1.1 Water Quality Monitoring/Parameters . . . . 15

3.1.2 Colilert Testing Reaction . . . . . . . . . . 16

3.1.3 Federal Water Quality Regulations . . . . . . . . 18

3.1.4 Virginia State Water Quality Regulations . . . . 19

3.2 Materials and Methods for Data Collection . . . . . . . 20

3.2.1 Sampling Locations . . . . . . . . . . . . 20

3.2.2 Sensor Calibration . . . . . . . . . . . . . . . 23

3.2.3 Data Collection Methodology . . . . . . . . . 26

3.2.4 Colilert Testing . . . . . . . . . . . . 27

3.2.5 Water Quality Results . . . . . . . . . . . 28

3.2.6 Colilert Testing Results . . . . . . . . . . 35

$3.3 \quad$ Discussion . . . . . . . . . . . . . . . 37

4 The Drone . . . . . . . . . . . . . . . . 40

4.1 Literature Review . . . . . . . . . . . . . . . . 40

4.2 Background Information . . . . . . . . . . . . . . 44

4.2.1 Unmanned Aerial Vehicles and their Use . . . . 44

4.2.2 Public Policy Surrounding Unmanned Aerial Ve-

hicles . . . . . . . . . . . . . 44

4.3 Materials and Methods for Drone Assembly . . . . . . . . 46

4.3.1 Physical Assembly of Drone . . . . . . . . . 46

4.3.2 Wiring of UAV Components . . . . . . . . . 48

4.3.3 Calibration of Drone . . . . . . . . . . . . . 54

4.3.4 Starting Up the UAV . . . . . . . . . . . 60

$4.4 \quad$ Results . . . . . . . . . . . . . . . . 62 
$4.5 \quad$ Discussion . . . . . . . . . . . . . . 63

5 Assembling the Wireless Sensor Network . . . . . . . . . . . . . 65

5.1 Background Information . . . . . . . . . . . . . 65

5.1.1 Wireless Sensor Networks . . . . . . . . . . 65

5.1.2 Systems Model Utilized in this Project . . . . . 67

5.2 Materials and Methods for Building the Wireless Sensor

Network . . . . . . . . . . . . . . . . . 68

5.2.1 Flashing Raspbian onto MicroSD Cards . . . . . 68

5.2.2 Enabling Remote Login to Raspberry Pi 3 . . . 69

5.2.3 Changing the Passwords on the Raspberry Pi Devices ................. 70

5.2.4 Setting up the Raspberry Pi 3 as an Access Point 71

5.2.5 Downloading and Editing the Arduino Code from the Atlas Website . . . . . . . . . . . 74

5.2.6 Wiring of the Temperature, $\mathrm{pH}$, and Conductivity Sensors to the Arduino . . . . . . . . . 75

5.2.7 Establishing Communication Between the Arduino and Raspberry Pi 0 . . . . . . . . . . 79

5.2.8 Establishing Communication Between the Raspberry Pi 0 and Raspberry Pi 3 . . . . . . . 81

5.2.9 Physical Construction of WSN Components . . . 82

5.3 Results . . . . . . . . . . . . . . 85

5.4 Discussion . . . . . . . . . . . . . . . 87

6 Future Work . . . . . . . . . . . . . . . . . . . . . 89

7 Acknowledgments . . . . . . . . . . . . . . . . . . . 91

8 Lab References . . . . . . . . . . . . . . . . . . . . . . 92

9 Appendices ....................... 96

9.1 Appendix A: Arduino I2C pH, Temperature, and Conduc-

tivity code . . . . . . . . . . . . 96

$9.2 \quad$ Appendix B: Arduino to Pi0 Python Code . . . . . . . . . 98

9.3 Appendix C: Python Code for Socket Coding Between Pi0 and $\mathrm{Pi} 3 \ldots \ldots \ldots \ldots$. . . . . . . . . . . . 99

9.4 Appendix D: Geographical Information System . . . . . 100 


\section{List of Figures}

1 Map of fish consumption advisories in the time following the discovery of Mercury in Waynesboro. Boones Run is located east of Elkton $[6] \ldots \ldots \ldots \ldots$

2 Map of the projects funded as a result of the DuPont Settlement Virginia. . . . . . . . . . . . . . . . . . 11

3 Google Earth screenshot displaying the location of Boones Run, outlined in purple. . . . . . . . . . . . . . . 12

4 Trail head of Boones Run, in George Washington National Forest. 13

5 Colilert coliform reaction. . . . . . . . . . . . . . . 16

6 Colilert E. coli reaction. . . . . . . . . . . . . . . . . . . 17

7 Map of the United States with all sources of surface water, including rivers, streams, and lakes, demarcated in blue. Terrestrial land is represented in yellow $[20] \ldots \ldots \ldots$. . . . . . . 18

8 Visual representation of the four sampling sites where water quality was tested throughout the duration of the project. . . . . . 20

9 Downstream photograph of Site 1 (left) and upstream photograph of Site 1 (right). . . . . . . . . . . . . . . . . 21

10 Upstream photograph of Site 2 (left) and a culvert at Site 2 (right). 22

11 Downstream photograph of Site 3 (right) and upstream photograph of Site 3 (left). . . . . . . . . . . . . . . . . . 22

12 Downstream photograph of Site 5 (right) and upstream photograph of Site 5 (left). . . . . . . . . . . . . . . . 23

13 Image of WTW meter used during site visits, including the handheld data display device and solutions to calibrate the $\mathrm{pH}$ probe and a saline solution for probe storage. . . . . . . . . . . 24

14 Conductivity probe used during testing. . . . . . . . . . . . 24

$15 \mathrm{pH}$ probe used during testing, which includes a silicone lid containing a saline solution to maintain integrity of the probe. . . . 25

16 DO sensor used during testing, including a protective capsule to ensure the probe remains moist in storage. . . . . . . . 26

17 Materials used to complete a Colilert coliform and E. coli test. . 28

18 Average DO concentration $(\mathrm{mg} / \mathrm{L})$ at Sites $1,2,3$, and 5. The red horizontal line at $6.0 \mathrm{mg} / \mathrm{L}$ indicates that water with $\mathrm{DO}$ concentrations less than $6.0 \mathrm{mg} / \mathrm{L}$ will not sustain brook trout. . 30

19 Average DO percent saturation from Sites 1, 2, 3, and 5. . . . 31 
20 Average water temperature $\left({ }^{\circ} \mathrm{C}\right)$ over time at Sites $1,2,3$, and 5. 32

21 Air temperature $\left({ }^{\circ} \mathrm{C}\right)$ over time. . . . . . . . . . . . . . 33

22 Average conductivity $(\mu \mathrm{S} / \mathrm{cm})$ at Sites $1,2,3$, and 5. . . . . . 34

23 Average stream pH over time at Sites 1, 2, 3, and 5. The red horizontal line at $\mathrm{pH} 9$ represents the upper bound of tolerable conditions for brook trout, while the red horizontal line at $\mathrm{pH} 6$ represents the lower bound of tolerable conditions [23] . . . . . 35

24 Bar chart illustrating the geometric means of coliform and E.coli concentrations at each site. . . . . . . . . . . . . 36

25 The WSN constructed to monitor a marine-coastal environment, components of which included environmental sensors placed upon buoys, a fixed-wing drone to collect the data, and a groundstation to which data was transmitted [26]. . . . . . . . . . . . . 40

26 Schematic representing data flow from the sensor nodes upon buoys to the control node located on the UAV and finally to a control node at a ground station [26] . . . . . . . . . . . . . .

27 Illustrated the design of a solar powered WSN and a UAV integrated into a data management platform for continuous monitoring of pollutant gases used by Malaver et. al [27]. . . . . . . . . .

28 Geo-location of nodes the UAV collected data from Malaver et. al, 2015 [27]. . . . . . . . . . . . . . . . . . . . . 43

29 This figure illustrates how the landing legs were attached to the UAV. . . . . . . . . . . . . . . . . . . . 46

30 Correct propeller attachment on the drone. The red arms are the front of the UAV and the white arms are the back. . . . . . . . . 47

31 The Raspberry Pi 3 mounted to the undercarriage of the UAV. .

32 Arrow on the Pixhawk flight controller that points to the front of the UAV. . . . . . . . . . . . . . . . . . . . . . . . . 49

33 Port on the Pixhawk controller for the safety switch. . . . . . . 49

34 Port on the Pixhawk for the buzzer. . . . . . . . . . . . . . 50

35 Port on the Pixhawk for the APM power module. . . . . . . . . . 50

36 Four main out slots on the Pixhawk controllers into which the motors were plugged [33]. . . . . . . . . . . . . . 51

37 This figure illustrates the numbers of the main out slots on the Pixhawk into which the corresponding motors were plugged [33]. 51

38 GPS module secured via epoxy to the GPS stand on the UAV. . 52

39 GPS module plugged into the GPS port (circled in red) on the Pixhawk. The arrow on the GPS module pointed in the same direction as the arrow on the Pixhawk (illustrated by the yellow circles). . . . . . . . . . . . . . . . 52

40 Location on the Pixhawk into which the receiver was plugged. . . 53

41 Image of the bind plug that got plugged into the Pixhawk. . . . 53

42 Illustrates the location on the Pixhawk into which the bind plug was inserted. . . . . . . . . . . . . . . . . 54

43 The Micro-USB port on the side of the Pixhawk. . . . . . . . . 55 
44 This figure illustrates the mandatory hardware configurations on Mission Planner required to setup and calibrate the UAV. . . . 56

45 Selecting the frame class and frame type on Mission Planner corresponding to the UAV. . . . . . . . . . . . . . 57

46 Green LED light on the Pixhawk to indicate it had a GPS lock. . 58

47 Locating the FailSafe mechanism within Mission Planner. . . . . 59

48 Configuring the FailSafe mechanism within Mission Planner. . . . 59

49 Correct way to plug the battery in to be charged. . . . . . . . . 60

50 Blue LED on the Pixhawk blinking to indicate the UAV is ready to arm. . . . . . . . . . . . . . . . 61

51 Image illustrating the location the left control stick on the remote control must be in to arm the UAV for flight. . . . . . . . . . . 62

52 Image of the bottom of a comparable DJI Phantom UAV [33]. . . 63

53 Two basic WSN Components: the sensing system as well as the data processing and storage component [40]. . . . . . . . . 65

54 Image depicting a flat topology. As seen in the image, the dark green circles that represent the nodes all play an equal part and are able to send information back and forth amongst each other [40]. . . . . . . . . . . . . . . . . . 6 66

55 Image illustrating a clustered topology. As seen in the image, the light green circles are only connected to one head-node (in dark green) and send information to that node alone. The head-nodes are capable of communicating with each other and forwarding the information received from the other nodes connected to it [40]. . 67

56 Data flow diagram illustrating flow of information from initial sensor to the end user. . . . . . . . . . . . . . 68

57 Lines that were added to the end of the hostapd. . . . . . . . 73

58 Lines that were added to the end of the rc.local file. . . . . . . 74

59 Initial wiring of the temperature, $\mathrm{pH}$, and conductivity circuits to the Arduino Uno to convert to using the $\mathrm{I}^{2} \mathrm{C}$ protocol [45]. . . 76

60 Connection of the TX to the PGND on the temperature and $\mathrm{pH}$

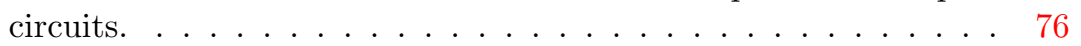

61 Connection of the TX to the rightmost PRB on the conductivity circuit. . . . . . . . . . . . . . . . 77

62 Wiring diagram of the three circuits soldered to the PCB and connected to the Arduino Uno. . . . . . . . . . . . . . . 78

63 Close-up image of the circuit board with the sensors connected to the Arduino Uno. . . . . . . . . . . . . . . . . . . 79

64 Arduino connected to the Raspberry Pi 0 . The USB end of the cord connected to the Arduino was plugged into a splitter that connects to the Raspberry Pi . . . . . . . . . . . . . . 80

65 Command that was placed in the /etc/rc.local file to get the arduino_pi01.py code to start on boot up of the Raspberry Pi 0. . 81

66 Image of the sensors and MircoUSB-to-USB cord fed through the hole drilled into the Pelican box. . . . . . . . . . . . . . . 83 
67 View of the hole drilled into the Pelican box and gauge fitted to the hole. . . . . . . . . . . . . . . . . 84

68 Image of the components inside of the Pelican box. . . . . . . . 85

69 Data wirelessly gathered from the ground-based node displayed on the Raspberry Pi 3. . . . . . . . . . . . . . . . . 86

70 Comparison of power consumption of small handheld devices in comparison to the ground based sensor node created for this project. 87

71 Arduino code controlling the three sensors. . . . . . . . . . . 96

72 Arduino code controlling the three sensors. . . . . . . . . . . 96

73 Arduino code controlling the three sensors. . . . . . . . . . . 97

74 Arduino code controlling the three sensors. . . . . . . . . . . 97

75 Arduino code controlling the three sensors. . . . . . . . . . . 97

76 Arduino code controlling the three sensors. . . . . . . . . . 98

77 Python code controlling the communication between the Arduino and Raspberry Pi $0 . \ldots \ldots \ldots$. . . . . . . . . . . 98

78 Python code that went in the client3.py file on the Raspberry Pi 0. 99

79 Python code that goes in the server.py file on the raspberry pi 3. 100

80 First screen of the story map created for this project. . . . . . . . 101 


\section{List of Tables}

1 GPS coordinates of the 4 sampling locations used during this phase of testing. . . . . . . . . . . . . . . . . . . . . . 21

2 The average DO concentration and standard deviation $(\mathrm{mg} / \mathrm{L})$ at Sites 1, 2, 3, and 5. . . . . . . . . . . . . . 29

3 The average DO percent saturation and standard deviation at Sites 1, 2, 3, and 5. . . . . . . . . . . . . . 30

4 The average change in water temperature and standard deviation

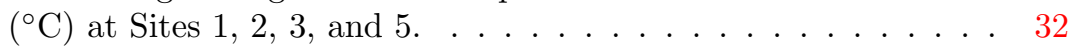

5 The average conductivity and standard deviation $(\mu \mathrm{S} / \mathrm{cm})$ at Sites 1, 2, 3, and 5. . . . . . . . . . . . . . . 33

6 The average pH and standard deviation at Sites 1, 2, 3, and 5. . 34

7 Coliform and E.coli concentrations measured using a MPN Chart. 36

8 Voltage, current, and power drawn from the ground-based node collecting data. . . . . . . . . . . . . . . . . 86 


\section{Abstract}

Water quality monitoring traditionally occurs via resource intensive field surveys, such as when a researcher manually collects data in a stream. Limiting factors such as time, money, and accessibility often result in less oversight of impaired water bodies, significantly threatening ecosystemic health and related ecosystem services. According to the United States Environmental Protection Agency, $84 \%$ of rivers and streams within the United States remain unassessed, resulting in significant lapses in available data [1]. Such lapses prohibit efficient and effective monitoring, restoration, and conservation efforts throughout the United States. The objective of this project was to employ an unmanned aerial vehicle to remotely collect data regarding water quality from a wireless sensor network. The site under analysis was Boones Run, a tributary of the South Fork of the Shenandoah River near Elkton, Virginia. This project served as a proof-of-concept that communication with a wireless sensor node has the capability to be deployed to collect data in remote areas efficiently and effectively. This system would be useful in areas where accessibility is difficult, and transmission of data for processing is not readily available due to the lack of network connectivity. Initial analysis of environmental data gathered by hand indicated that surrounding land use had a significant impact on Boones Run water quality. This conclusion was reached given the trends seen in dissolved oxygen, water temperature, $\mathrm{pH}$, and conductivity data from upstream to downstream over time. The completion of this project also lead to the successful data flow amongst all parts in the wireless sensor network. Three sensors soldered to a breadboard and connected to an Arduino Uno were able to gather data and send it to a Raspberry Pi 0 . The Raspberry Pi 0 acted as a temporary storage device for the data before it was sent wirelessly to a Raspberry Pi 3 acting as an access point. The Raspberry Pi 3 device was mounted to an unmanned aerial vehicle so it could be flown over the node to decrease data collection time as well as adding the ability to collect data from places that are otherwise difficult for humans to access. 


\section{Introduction to Environmental Monitoring and Regional Context}

\subsection{DuPont Mercury Contamination of the South River in Waynesboro, Virginia}

Water quality refers to the state of physical, chemical, and biological characteristics of water. Water quality monitoring is the process through which data relating to these characteristics is amassed. Water quality data can be monitored via several metrics, including temperature, dissolved oxygen (DO) concentration and percent saturation, conductivity, and $\mathrm{pH}$. Empirical data is used to ensure compliance with legislation such as the Clean Water Act (CWA) and Safe Drinking Water Act. In the case of non-compliance, analysis of water quality data is used to inform restoration practices [2]. Boones Run, a tributary to the South Fork of the Shenandoah River, was selected as the site to implement a wireless sensor network (WSN) due to the possibility of impairment. Land adjacent to Boones Run was awarded to the Virginia Department of Forestry for stream restoration following a settlement with DuPont regarding contamination of the South Fork of the Shenandoah River.

In 1929, DuPont established a manufacturing plant in Waynesboro, Virginia. The plant, located in an industrially zoned area, occupies 153 acres of land [3]. The plant produced yarn, Orlon, and Rayon, which was the world's first synthetic fiber. Mercury was used as a catalyst during the production process of turning acetate flakes into Rayon Fiber [4]. Despite opening operations during the Great Depression, the DuPont factory expanded its production systems at a rapid rate, resulting in an a large quantity of unregulated byproducts being disposed of in the South River. Until 1950, the company utilized mercury to manufacture Rayon. Whilst in the midst of construction activities in 1976, DuPont discovered high mercury concentrations within the soil, and began conducting studies to determine how mercury contamination may have impacted the immediate waterways and greater watershed [5]. In 2008, the South River Science Team extensively sampled floodplain soil alongside the South River; $7 \%$ of samples had mercury concentrations greater than 23 parts per million [3]. Background levels of mercury within soil do not typically exceed one ppm.

In the year following DuPont's initial discovery of mercury contamination, the Virginia Department of Health instituted a fish consumption ban from Waynesboro to Front Royal. This consumption ban impacted major waterways such as the South River, South Fork Shenandoah River, and part of the Shenandoah River [3]. Mercury is a neurotoxin, and may negatively impact organisms, such as fish, that ingest it. Mercury bioaccumulates as it moves up the food chain, placing people that ingest organisms such as fish at a high risk. Consumption bans within the South Fork of the Shenandoah River cautioned against consuming more than one pound of fish sourced from the river per month, particularly 
for pregnant and nursing women and young children. Mercury levels within the South River and South Fork of the Shenandoah River were not high enough to threaten recreational activities, such as swimming, boating, and wading within the water [5]. Figure 1 highlights the extent to which fish bans were implemented following the discovery of mercury.

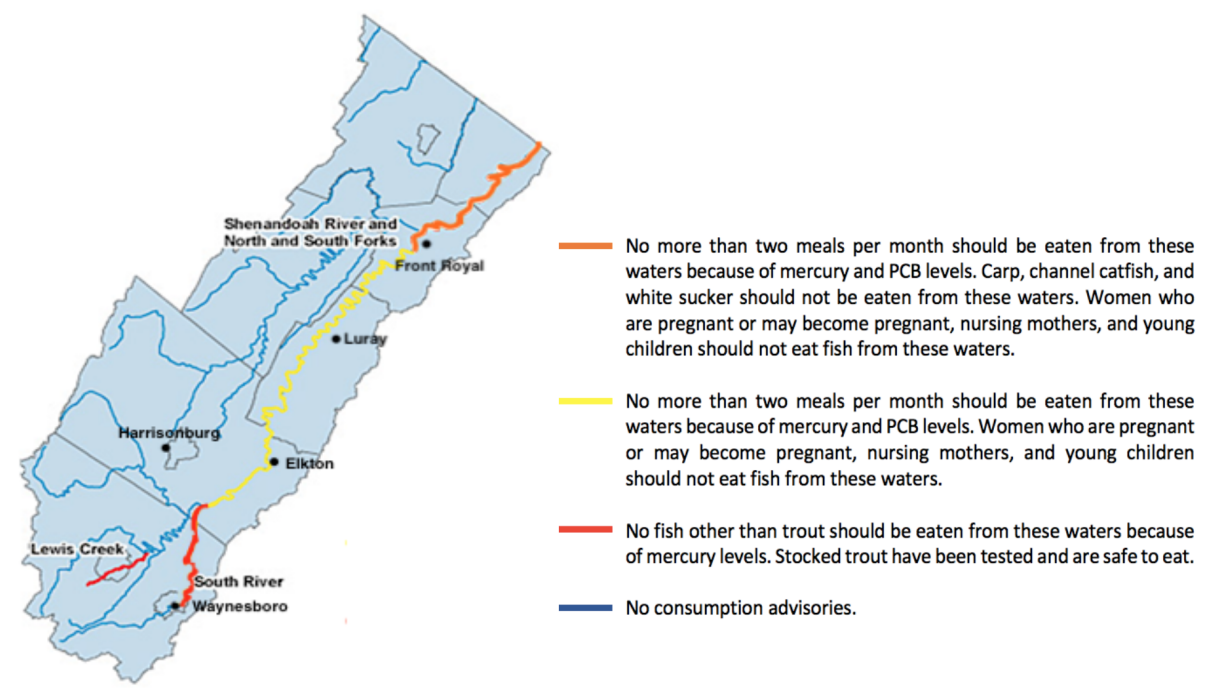

Figure 1: Map of fish consumption advisories in the time following the discovery of Mercury in Waynesboro. Boones Run is located east of Elkton [6].

The high levels of mercury stemming from the DuPont contamination have contributed to an impaired state of the greater watershed. DuPont released mercury into the river for 21 years before their manufacturing production changed and the use of mercury ceased. During this time, it is estimated that thousands of gallons of mercury were dumped into the river, completely unregulated. The negative effects have been seen across miles of rivers, adversely impacting wildlife [7]. The United States (U.S.) Fish and Wildlife Service in conjunction with the Secretary of Natural Resources worked with DuPont to identify the impacts of the mercury spillage. On July 28, 2017, a $\$ 42$ million dollar settlement was approved by the U.S. District Court for the Western District of Virginia to fund restoration projects in and around the area, at sites that mitigate the ecological impact of the mercury from the DuPont contamination [7]. Figure 2 is a map which highlights some of the local projects that are receiving funding as a result of the DuPont settlement. 


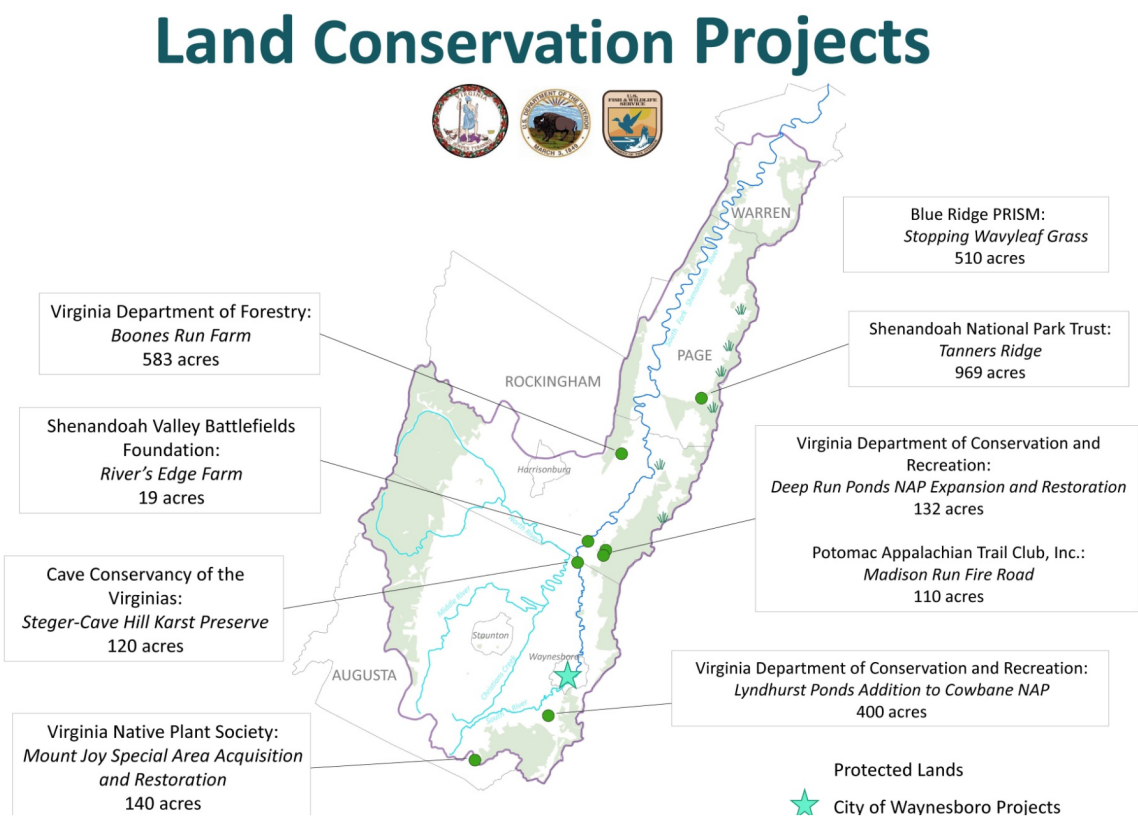

Figure 2: Map of the projects funded as a result of the DuPont Settlement Virginia.

\subsection{Boones Run}

Boones Run is a small stream located on the eastern side of the Massanutten Mountains in Rockingham County, Virginia. The property as of April 3, 2019 is in the process of switching ownership from private land to state property awarded to the Virginia Department of Forestry. The stream is located in the South Fork Shenandoah Watershed, and is a tributary to the South Fork of the Shenandoah River. Figure 3 provides a Google Earth screenshot displaying the location of Boones Run, outlined in purple. The Elkton airport, now out of commission, is symbolized by an airplane to the right of the stream. 


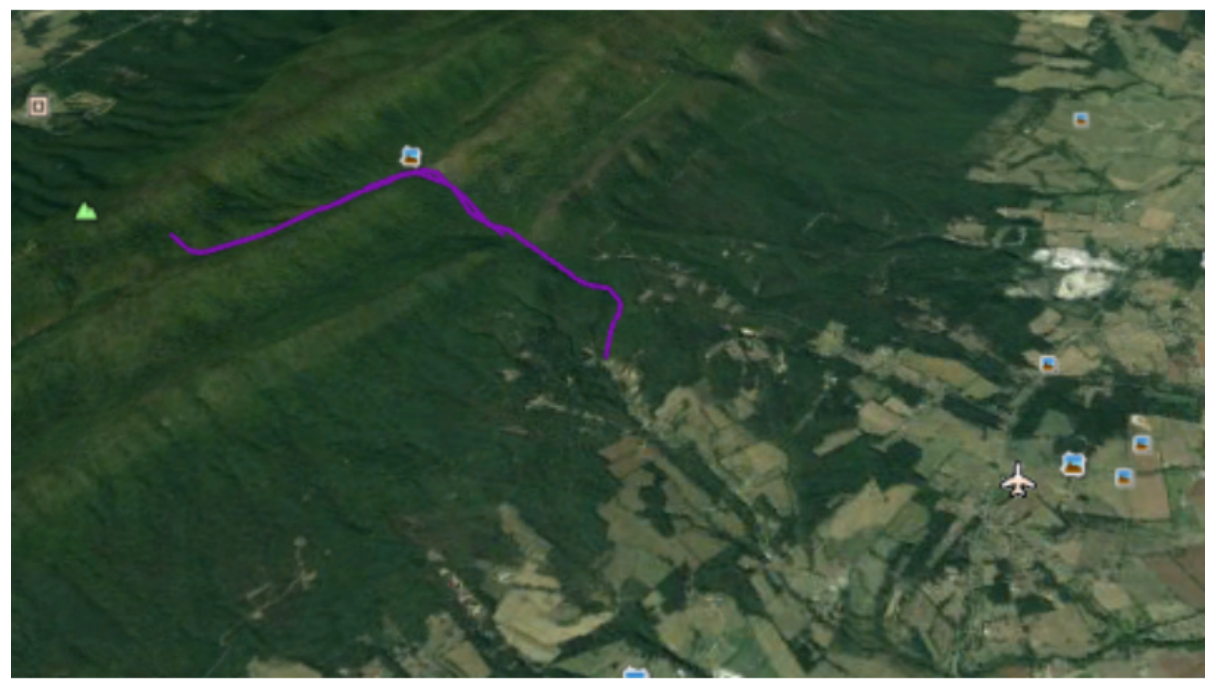

Figure 3: Google Earth screenshot displaying the location of Boones Run, outlined in purple.

The stream is approximately a 40 minute drive from James Madison University, depending on the exact testing location. Approximately 11.5 square miles drains into Boones Run [8]. While Boones Run itself is not contaminated with mercury, this site is receiving funding to be restored as an offset of the ecological impacts of mercury on the South River and South Fork of the Shenandoah River. There are numerous endangered species that inhabit the area, including the Shenandoah salamander and a wildflower known as the Virginia sneezeweed [9]. The primary species of concern is the eastern brook trout, a key indicator of environmental health [10]. 


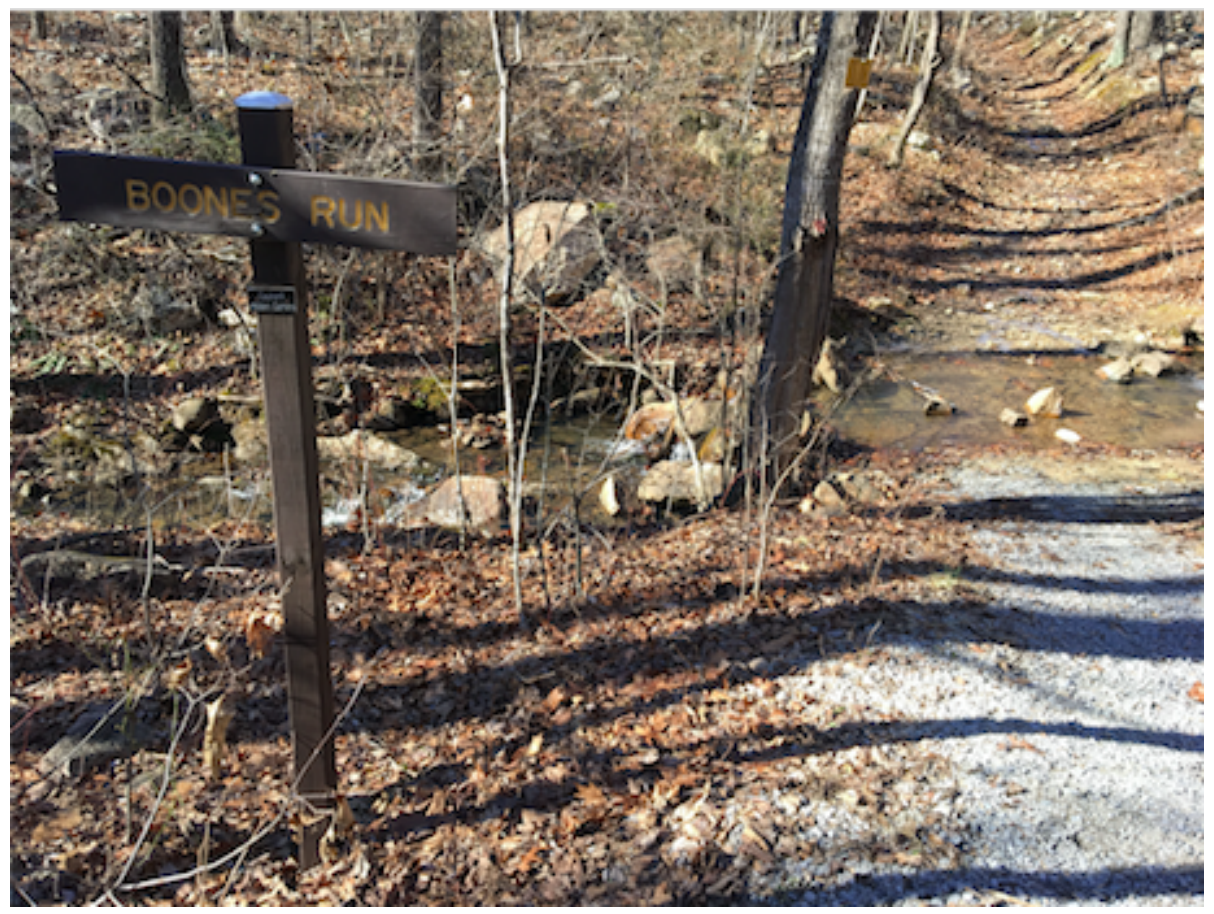

Figure 4: Trail head of Boones Run, in George Washington National Forest.

\subsection{Public Opinion Regarding the Impairments of the South River}

Waynesboro was, and is still to this day, highly industrialized and replete with families working to earn a living and enjoy a healthy lifestyle. Numerous families living within the area have resided there for generations and raise families of their own. Back in the 1920s, Waynesboro residents avoided swimming in and/or eating products sourced from the river. Waynesboro residents suspected dumping into the river, as the river changed colors based on what the corduroy plant manufactured day by day. Livestock throughout the Shenandoah Valley also contaminated waterways with waste. People avoided recreating within the river due to apprehensions regarding contamination [11].

Along with recreational activities, fish have been a large concern to local residents. Many people exercise their right to fish however they often release them due to concerns of mercury contamination. Other locals refuse to eat the fish because they do not support or like the taste of stocked fish as opposed to wild fish. After the discovery of Mercury, a fish ban was implemented for miles along the South River. While it was later reduced to a consumption advisory, people still tread lightly and take precaution if they eat fish that are bred in the river [11]. 


\subsection{Industrial Norms of Non-Regulated Disposal of Ma- terial in the South River}

The South River has been subject to a number of preventable incidents in recent history, many of which occurred as a result of inadequate water quality monitoring and waste disposal. DuPont discharged mercury into the South River for 21 years with little to no oversight to prevent the discharge. Many companies took advantage of the lack of regulation and dumped pollutants into the river. Many people avoided recreational activities (such as swimming) in the South River, long before it was known that mercury and other hazardous substances were being dumped into the river [11]. Waynesboro opened a wastewater treatment plant in 2010 [12]. Prior to the opening of the treatment plant, there was unregulated waste dumping into the South River. The river served as an outlet for substance disposal that people and companies alike used to their advantage. The lack of awareness to the future ramifications were widely unknown and river remains impaired 100 years later. 


\section{$3 \quad$ Water Quality and Data Collection}

\subsection{Background Information}

\subsubsection{Water Quality Monitoring/Parameters}

For this project, temperature, dissolved oxygen (DO), conductivity, and $\mathrm{pH}$ sensors will be utilized, as well as Colilert coliform and E.coli testing to monitor the water quality of Boones Run. Water temperature impacts the concentration of oxygen that can be dissolved in water, the rate at which algae and other aquatic plants undergo photosynthesis, the metabolic rate of organisms, and the sensitivity of species to parasites and diseases [13]. Water levels in a river can impact the vegetation and riparian health of the stream. Vegetation growth can be greatly impacted by abrupt change in water levels and lack of vegetation can result in stream degradation. Maintaining steady water levels throughout the year fosters a healthy environment for aquatic species and enables the surrounding ecosystem to flourish [14].

Commonly used water quality measures are $\mathrm{DO}$, conductivity, $\mathrm{pH}$, and coliform and E. coli bacteria count. DO is the measure of oxygen that is dissolved in water and is essential to the survival of many species. DO can be an indicator of species health such as trout, stoneflies, catfish worms and dragonflies. Insufficient concentrations of DO may impact the aforementioned species' reproductive habits and habitats, leading to population declines. According to the Virginia Legislative Code, the maximum sustainable temperature for brook trout living within natural trout waters is $68^{\circ} \mathrm{F}$. The minimum DO required to sustain brook trout is $6.0 \mathrm{mg} / \mathrm{L}$, with streams averaging $7.0 \mathrm{mg} / \mathrm{L}$ [15].

Conductivity measures the amount of substances dissolved into the water that conduct electricity. While geology and precipitation impact conductivity, high conductivity may be indicative of a problem, such as a failing sewage system discharging ions into rivers. Conductivity can impact not only human uses of stream water such as irrigating farmlands or providing drinking water, but also aquatic species sensitive to changes in conductivity. The United States Environmental Protection Agency (U.S. EPA) identifies most rivers as having a conductivity range of 50 to 1,500 micro-Siemens per centimeter $(\mu \mathrm{S} / \mathrm{cm})[15]$.

$\mathrm{pH}$ is a measure of the acidity or alkalinity of water. If the water becomes too basic or too acidic, it becomes hazardous to certain organisms. As many species often thrive in a sensitive range of $\mathrm{pH}$, an abrupt change can damage an aquatic ecosystem [13]. According to the Virginia Legislative Code, water with a $\mathrm{pH}$ range between 6 and 9 is optimal for brook trout survival within natural trout waters [15].

The existence of $E$. coli in water indicates the presence fecal coliform. While E. coli is detrimental to stream quality, it can denote the presence of other 
harmful pathogenic species. Fecal coliform bacteria are often detected in water bodies within the Shenandoah Valley due to the vast number of farms within the region. High levels of these pathogenic species are toxic to aquatic species [16].

\subsubsection{Colilert Testing Reaction}

Colilert testing makes use of of proprietary Defined Substrate Technology (DST) to simultaneously detect the total coliform and $E$. coli within a water sample. This reagent system is designed to quantify these two specific targets from a mixture of bacteria. The Colilert reagent packet consists of two carbon sources, nitrophenyl-beta-D-galactopyranoside (ONPG), and 4-methylumbeilliferyl-betaD-glucuronide (MUG), which when hydrolyzed produce a colored substrate. The coliform enzyme $\beta$-galactosidase metabolizes ONPG during the incubation period, and produces a yellow color as a byproduct as depicted in Figure 5. Simultaneously, the $\beta$-glucuronidase enzyme found in E. coli metabolized MUG, creating a fluorescent by-product visible under UV light, as seen in Figure 6 [17].

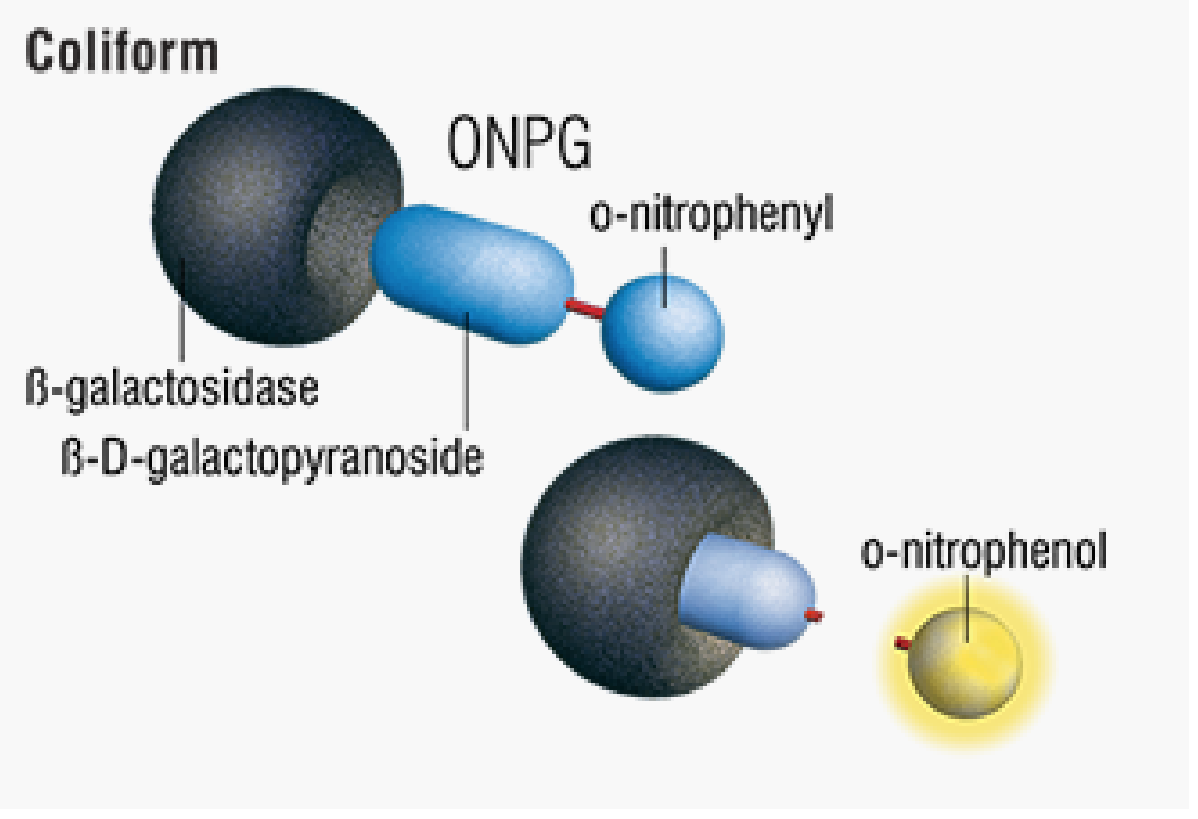

Figure 5: Colilert coliform reaction. 

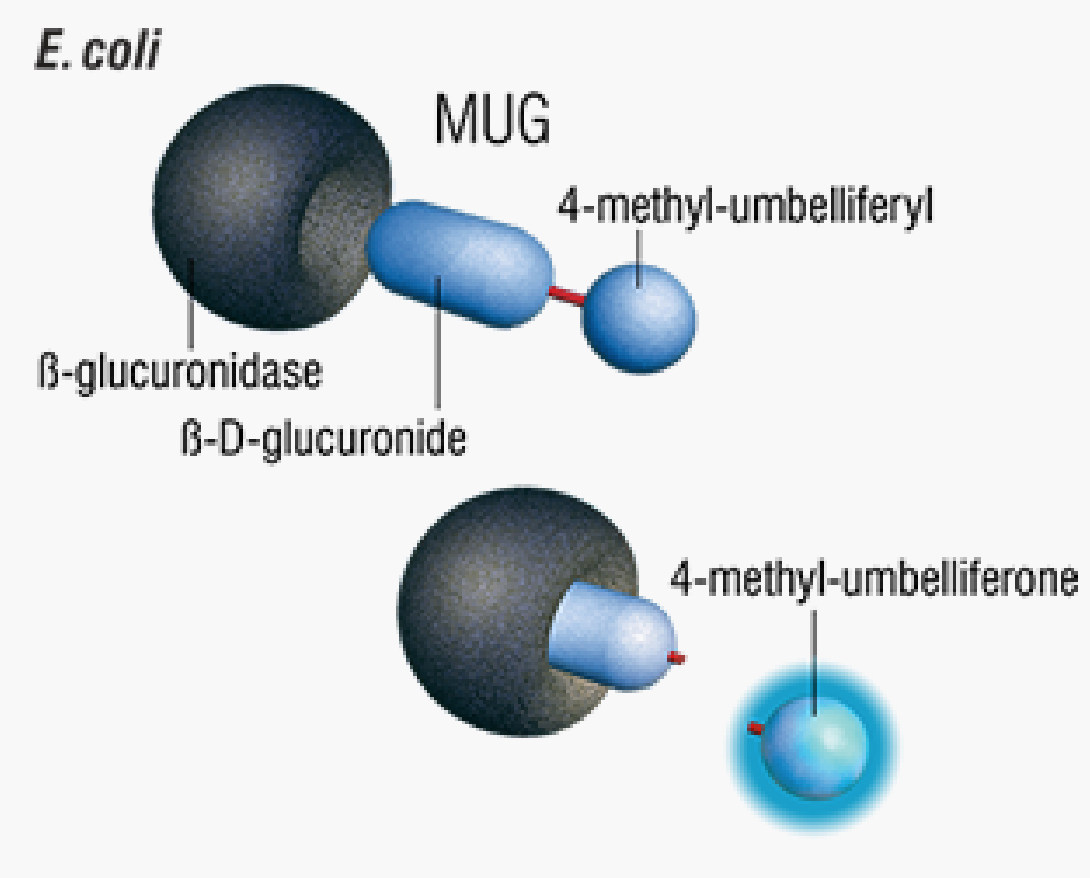

Figure 6: Colilert E. coli reaction.

Colilert differs from other water quality tests by inhibiting growth of nontargeted bacteria, therefore only allowing specific targets to grow during the incubation period. The selectivity factor of Colilert testing prevents the growth of non-specific targets, and subsequently potential false positives. Because these two enzymes are not typically found in non-coliform bacteria, no other bacteria within the sample would be able to produce a colored substrate. Colilert testing is EPA-approved as a test for drinking, wastewater, and source contaminated water. It is low cost, efficient, and produces highly accurate results in a short time period. [17]

Most Probable Number (MPN) tables [18] are used as a method to estimate concentrations of viable microorganisms in a water sample by means of tenfold dilutions. It is often used to measure microbial populations in soil, water, and agriculture products. While using this method does not give a definitive number of microbial populations of specific organisms, it is used as a safety determinant to ensure that bacteria do not surpass safe thresholds. This tool is advantageous for quick, broad data data collection, as in the case of this study. Trends are still observable despite not having definitive numbers, making this test advantageous over some of the more costly and time consuming tests for coliform and E. coli [19]. 


\subsubsection{Federal Water Quality Regulations}

A vast network of streams and rivers occupying a total length of approximately 3,533,305 miles transects the United States [1]. This network supplies habitats for a variety of organisms, serves as an integral food source for humans and animals alike, and provides an array of ecosystem services to society. Figure 7 displays all sources of surface water within the United States, including rivers, streams, and lakes.

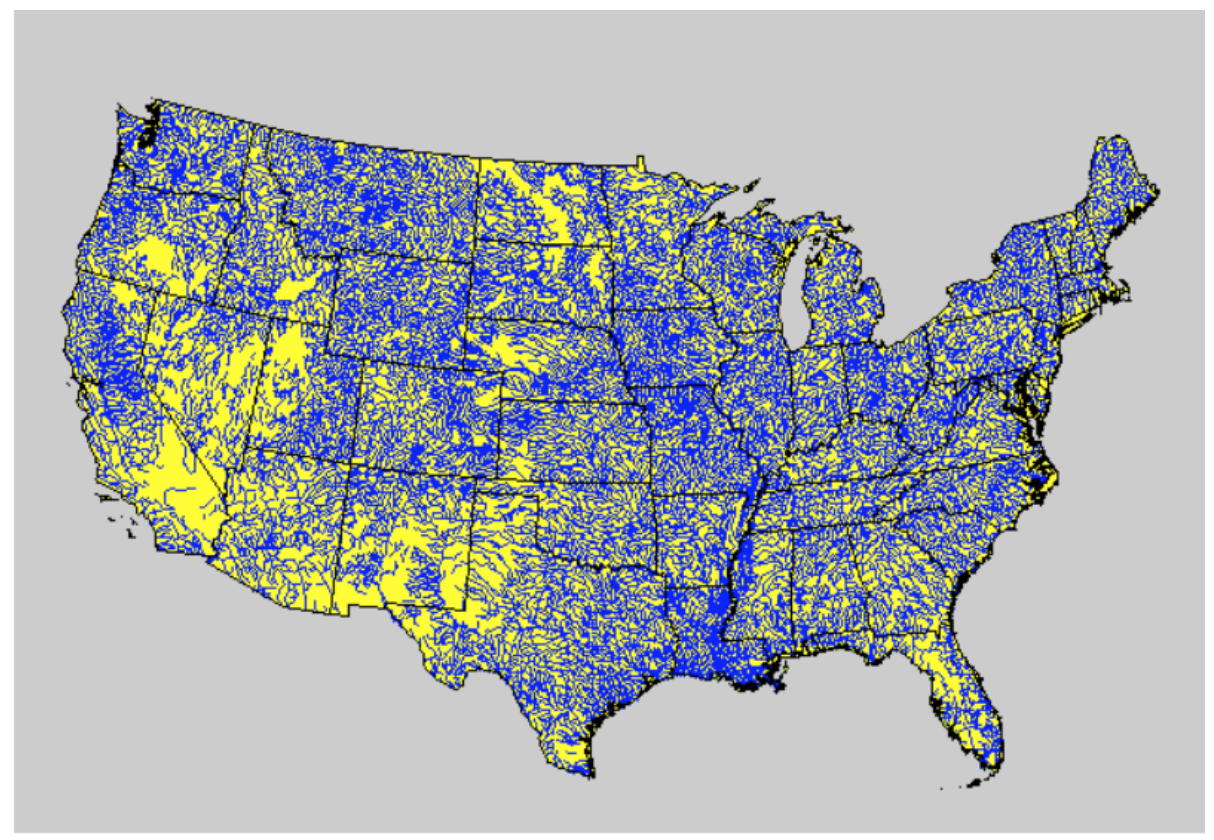

Figure 7: Map of the United States with all sources of surface water, including rivers, streams, and lakes, demarcated in blue. Terrestrial land is represented in yellow [20].

In an effort to preserve freshwater resources within the U.S that are under threat of pollution, the U.S. government passed the Federal Water Pollution Control Act of 1948. Following amendments and reforms, the Federal Water Pollution Control Act became known as the Clean Water Act (CWA) in 1972. The CWA primarily established infrastructure to regulate pollutant discharges into surface waters and provided the U.S. EPA with the authority to outlaw the discharge of point source pollutants without permits. Furthermore, the CWA implemented pollution control programs, funded the construction of sewage treatment plants to ensure the safe treatment and disposal of waste, and began the national conversation pertaining to monitoring non-point source pollution [21].

The CWA was further amended following initial revisions in an effort to contin- 
uously update and enhance water quality protection and monitoring within the U.S. In 1981, amendments streamlined and enhanced the construction of municipal sewage treatment plants. In 1987, the program through which sewage treatment plants were constructed was replaced with the State Water Pollution Control Revolving Fund (Clean Water State Revolving Fund), which continued to address water quality needs with the utilization of EPA-state partnerships [21].

Of the 3,533,205 miles of rivers that bisect the United States, only roughly 563,955 miles, or $16 \%$ of rivers, have been assessed by state, tribal, and local jurisdictions under the CWA [1]. Of the assessed waters, $44 \%$ of rivers were identified as impaired and $3 \%$ as threatened. Water bodies receive the designation "impaired" or "threatened" if contamination or degradation exceeds established standards. Standards are contingent upon designated water use, such as drinking or fishing [1]. For example, the U.S. EPA defines the reference concentration (RfC) of mercury to be $3.0 \times 10^{-4} \mathrm{mg} / \mathrm{m}^{3}$ [4]. Comprehensive data regarding the quality of rivers and streams within the U.S. is lacking due to time and monetary restraints. Particular attention may be paid to watersheds in which there is a known source of contamination, such as the mercury contamination of the South River and South Fork of the Shenandoah River in the Shenandoah Valley of Virginia.

\subsubsection{Virginia State Water Quality Regulations}

Although the U.S. EPA implemented the CWA federally, individual states also bear responsibilities to ensure the quality of waterways within the U.S. The Virginia Department of Environmental Quality (VADEQ) regulates pollution and the use of water resources through the State Water Control Board. These agencies issue permits to protect and maintain the quality of water, including Virginia Pollutant Discharge Elimination System (VPDES) permits, Virginia Stormwater Management Program (VSMP) permits, and Virginia Pollution Abatement permits. The National Pollutant Discharge Elimination System (NPDES) was established under Section 402 of the CWA, and limits pollutant discharges into streams, rivers, and bays. The VADEQ issues VPDES permits for point source discharges to surface waters, as well as discharge from industrial activities. Virginia Pollution Abatement permits regulate the treatment of sewage sludge, storage and application of biosolids, and industrial wastes. Additionally, Virginia Water Protection permits safeguard against impacts to wetlands and streams as a result of development and/or surface water withdrawals, and ground water withdrawal permits regulate groundwater withdrawals within specified groundwater management areas [22]. 


\subsection{Materials and Methods for Data Collection}

\subsubsection{Sampling Locations}

Figure 8 below represents the GPS coordinates of the sampling sites. BOONR01, BOONR02, BOONR03, and BOONR05 were the 4 sampling locations chosen. Site 4 was intentionally skipped as it has to the potential to be added in the future after land ownership changes between Sites 3 and 5 . There are approximately 0.80 miles between Sites 1 and 2, approximately 0.46 miles between Sites 2 and 3, and approximately 1.75 miles between Sites 3 and 5. As of April 3, 2019, the land was still in private hands and the experimenters did not have access to a location for Site 4 . Site 1 was shifted slightly upstream from the original location due to a tree falling in December 2018, rendering the original testing site inaccessible. The current Site 1 testing location is slightly upstream of the original site and above a five-foot waterfall. The change in testing coordinates was expected to have minimal effect on the water quality data.

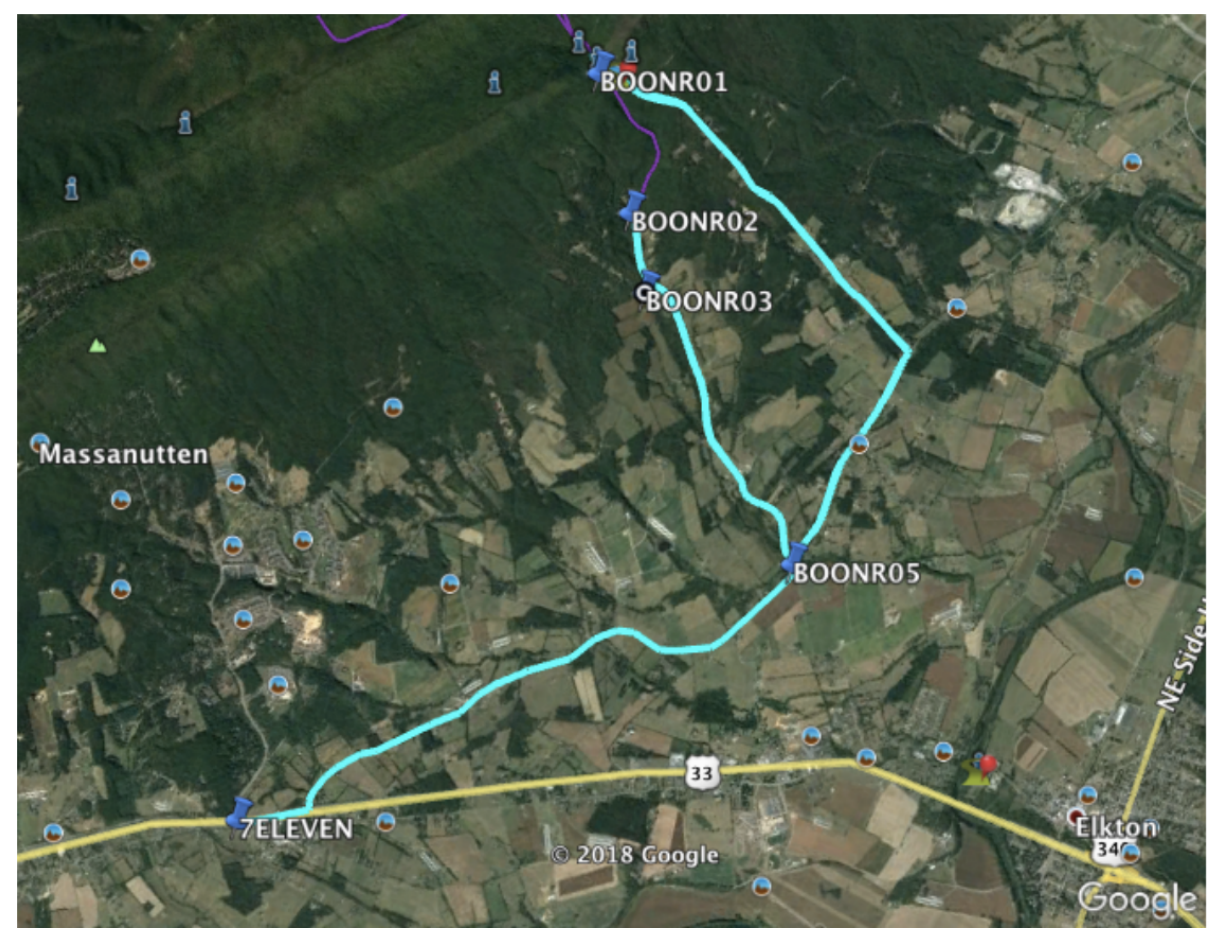

Figure 8: Visual representation of the four sampling sites where water quality was tested throughout the duration of the project. 


\begin{tabular}{ccc}
\hline Site & GPS Coordinates & Description of Site \\
\hline 1 & $38^{\circ} 27^{\prime} 10.12^{\prime \prime} \mathrm{N}, 78^{\circ} 41^{\prime} 44.16^{\prime \prime} \mathrm{W}$ & $\begin{array}{c}\text { Slight road widening near Boones Run trailhead off } \\
\text { Runkles Gap Road }\end{array}$ \\
1 & $38^{\circ} 27^{\prime} 10.55^{\prime \prime} \mathrm{N}, 78^{\circ} 41^{\prime} 44.87^{\prime \prime} \mathrm{W}$ & $\begin{array}{c}\text { Slight road widening near Boones Run trailhead off } \\
\text { Runkles Gap Road. Upstream of original Site 1 location } \\
\text { due to a tree blocking the path to the stream. }\end{array}$ \\
2 & $38^{\circ} 26^{\prime} 34.81^{\prime \prime} \mathrm{N}, 78^{\circ} 41^{\prime} 16.20^{\prime \prime} \mathrm{W}$ & Culvert at Bryant Hollow Road \\
3 & $38^{\circ} 26^{\prime} 14.10^{\prime \prime} \mathrm{N}, 78^{\circ} 41^{\prime} 01.65^{\prime \prime} \mathrm{W}$ & $\begin{array}{c}\text { Culvert near Mt Sinai Church } \\
5\end{array}$ \\
$38^{\circ} 25^{\prime} 12.32^{\prime \prime} \mathrm{N}, 78^{\circ} 39^{\prime} 35.85^{\prime \prime} \mathrm{W}$ & Culvert near intersection of Bryant Hollow Road and East \\
& & Point Road \\
\hline
\end{tabular}

Table 1: GPS coordinates of the 4 sampling locations used during this phase of testing.

Figure 9 is a downstream photograph of Site 1 (left) and an upstream photograph of Site 1 (right), located within the George Washington National Forest. Figure 10 is an upstream photograph of Site 2 (left) and a culvert at Site 2 (right). Figure 11 is an upstream photograph of Site 3 (left) and a downstream photograph at Site 3 (right). Figure 12 is an upstream photograph of Site 5 (left) and bridge at Site 5 (right).
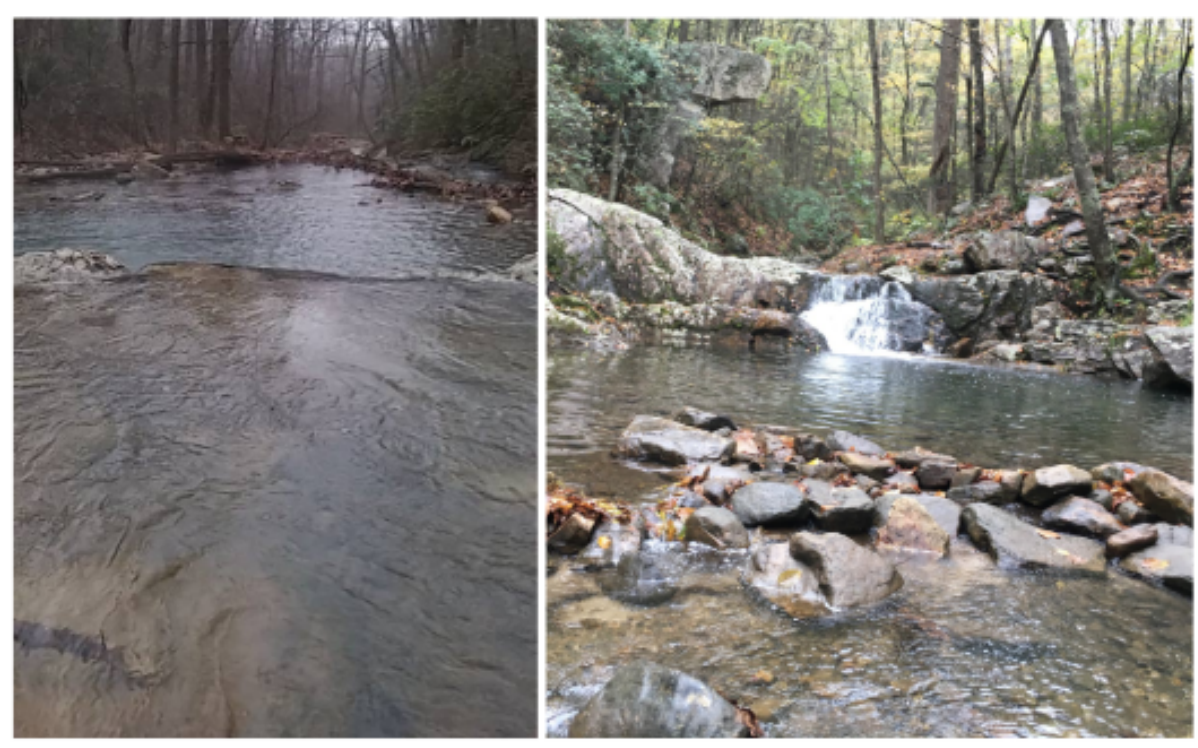

Figure 9: Downstream photograph of Site 1 (left) and upstream photograph of Site 1 (right). 


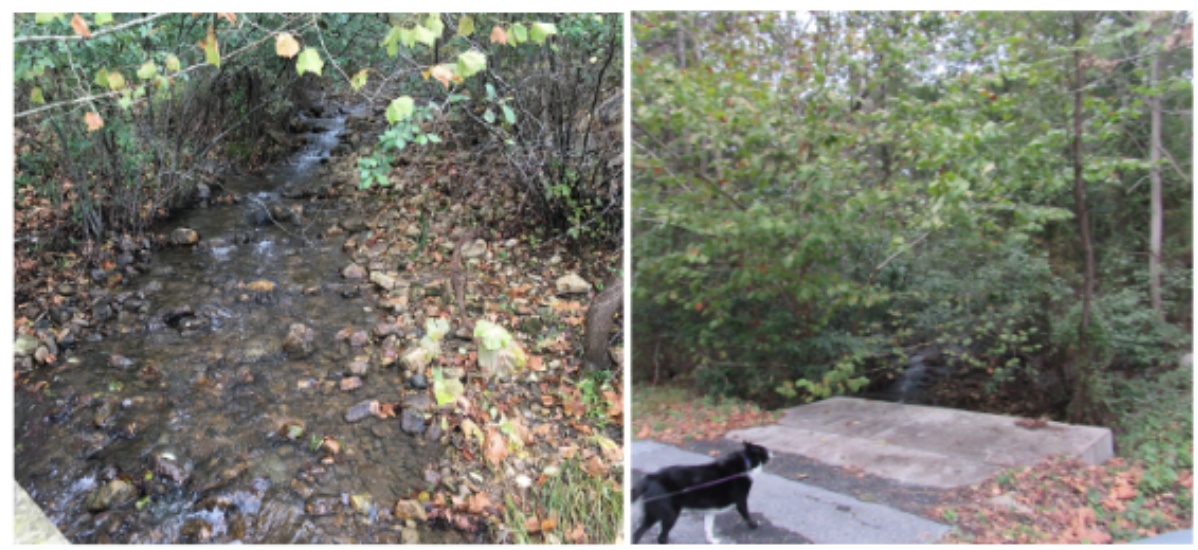

Figure 10: Upstream photograph of Site 2 (left) and a culvert at Site 2 (right).

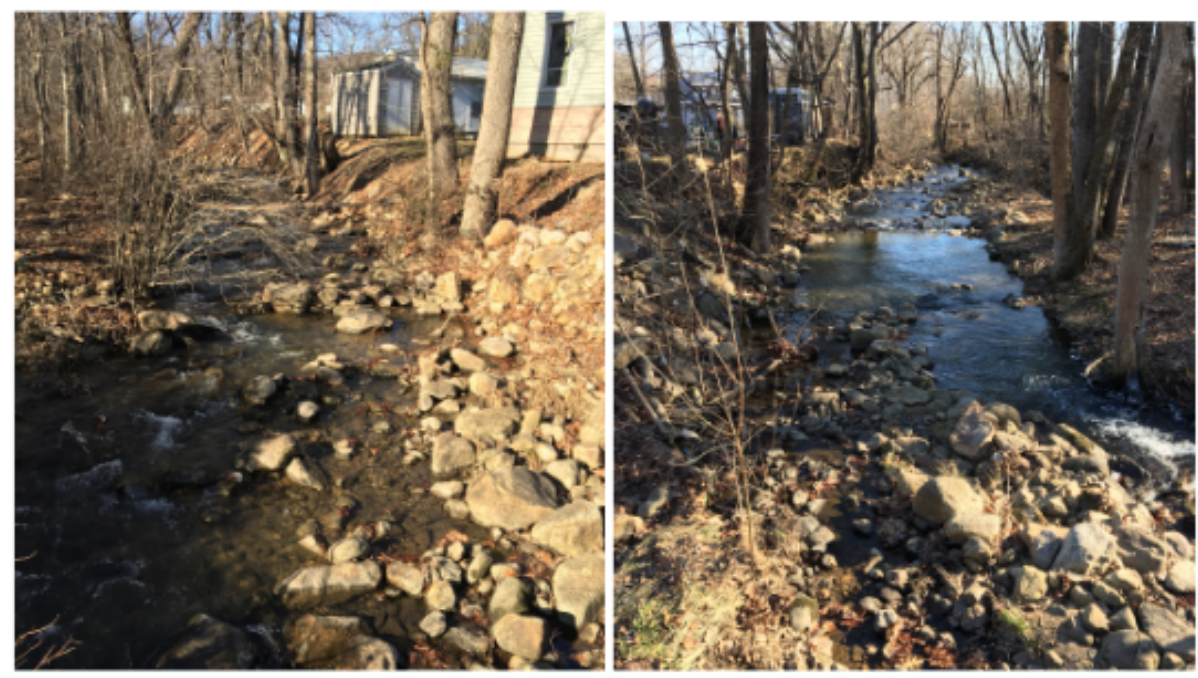

Figure 11: Downstream photograph of Site 3 (right) and upstream photograph of Site 3 (left). 

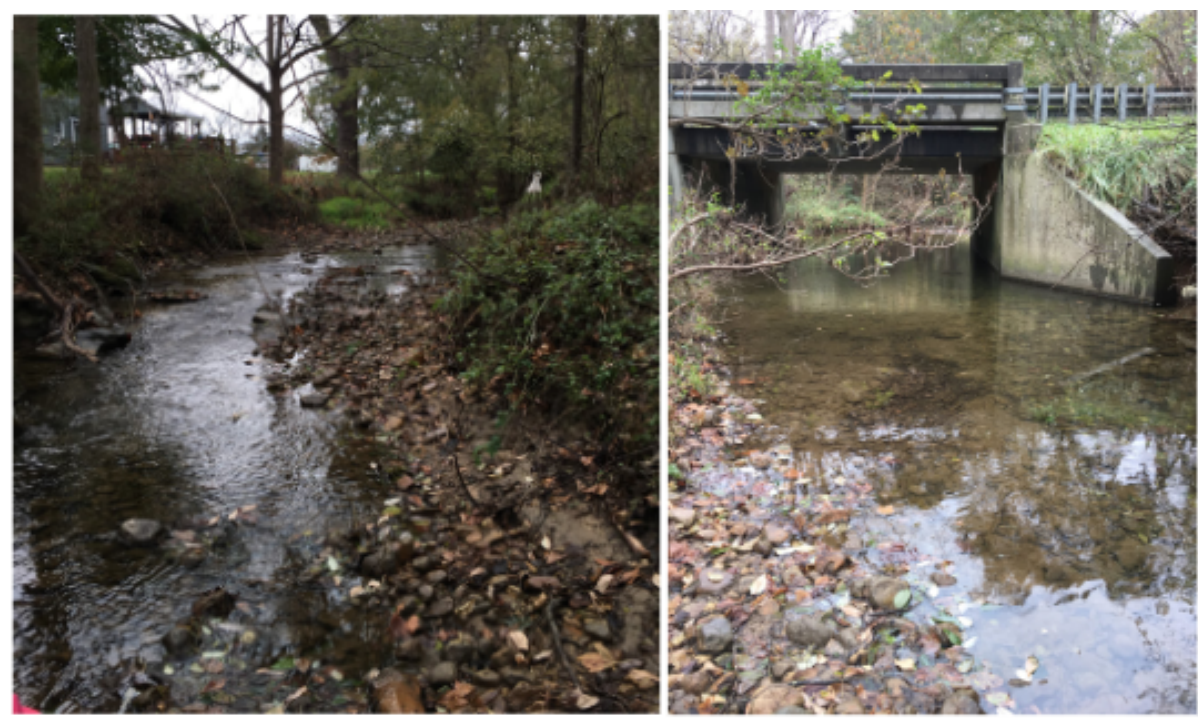

Figure 12: Downstream photograph of Site 5 (right) and upstream photograph of Site 5 (left).

\subsubsection{Sensor Calibration}

Before hand sampling could be executed, each of the sensors had to be calibrated to ensure that the readings would be accurate. It was not necessary to calibrate the sensors between every site visit, but if the readings took a significant amount of time to compile, or they were inconsistent, it was recommended to calibrate the sensors. During testing, a Wissenschaftlich- Technische Werkstätten (WTW) meter was used, as observed in Figure 13. The model used was a Multi 3400i / Set, and the probes used were DO, Conductivity, and pH. Water temperature was measured using the DO probe. Each probe used can be observed in Figures 14, 15, and 16. 


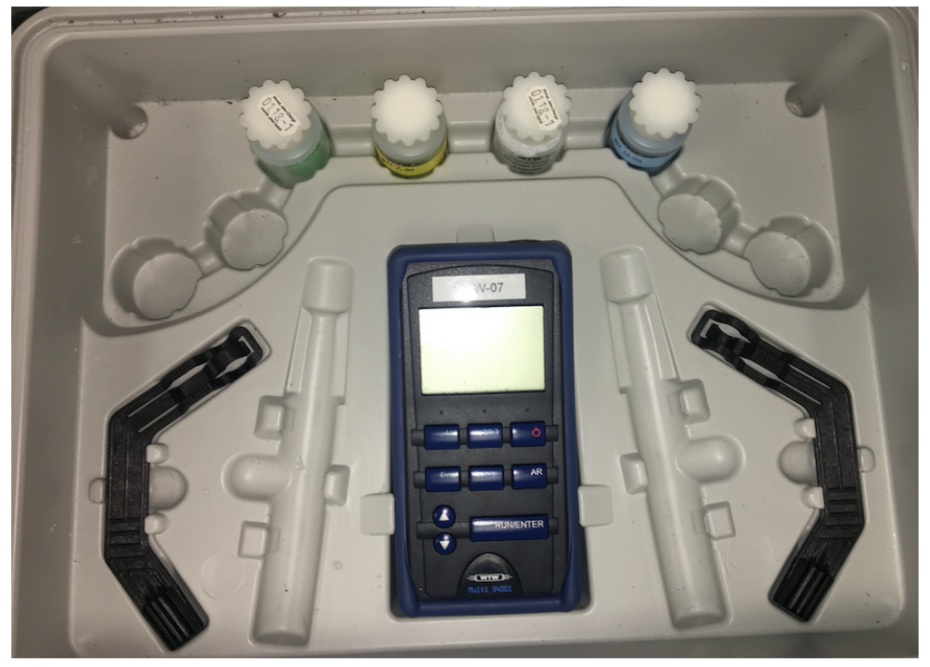

Figure 13: Image of WTW meter used during site visits, including the handheld data display device and solutions to calibrate the $\mathrm{pH}$ probe and a saline solution for probe storage.

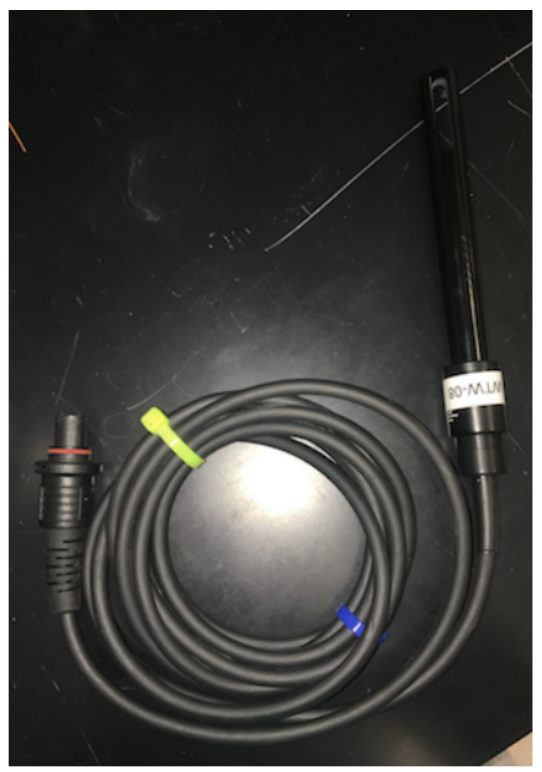

Figure 14: Conductivity probe used during testing. 


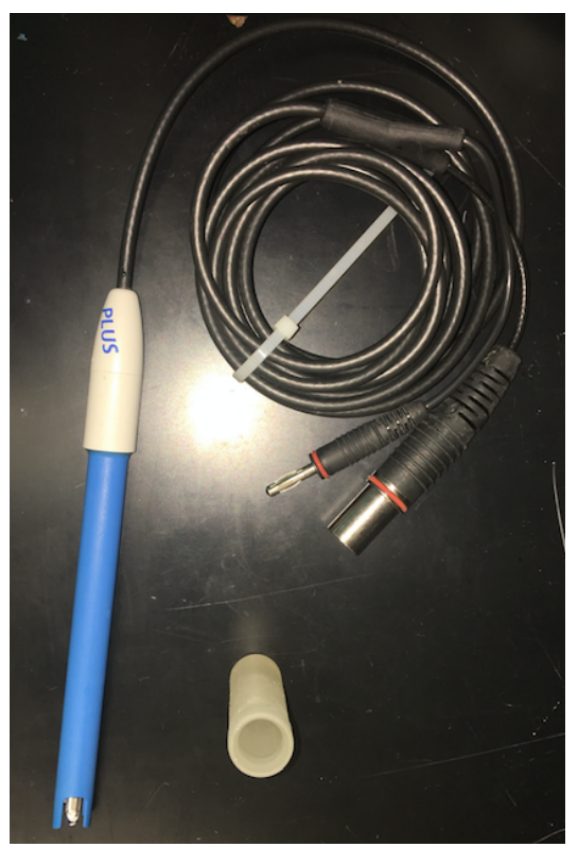

Figure 15: pH probe used during testing, which includes a silicone lid containing a saline solution to maintain integrity of the probe. 


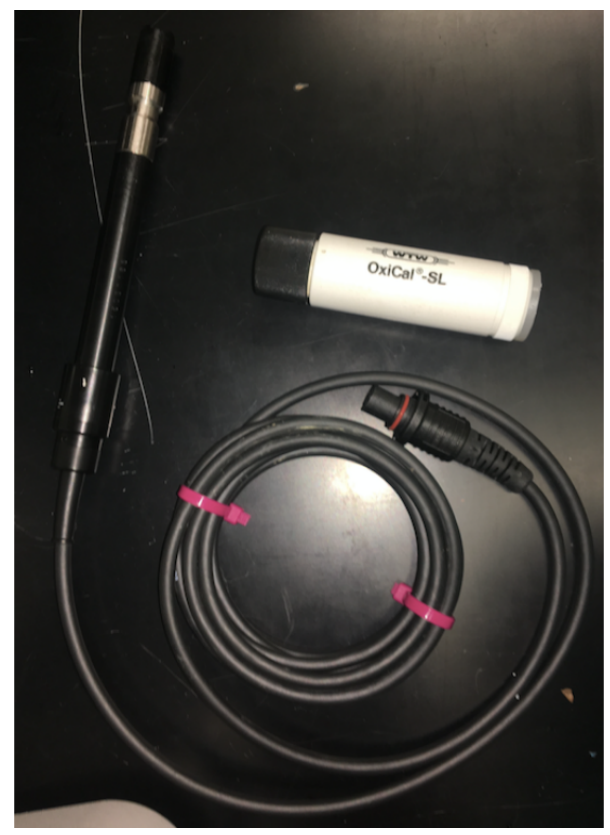

Figure 16: DO sensor used during testing, including a protective capsule to ensure the probe remains moist in storage.

In order to calibrate the DO sensor, the sensor first had to be connected to the WTW meter. The outer shell casing was removed from the sensor, and the sponge within was checked for moisture. The $\langle\mathrm{CAL}\rangle$ key was pressed, then the $<$ RUN/ENTER $>$ key. Calibration was finished once AR stopped flashing. Next, the conductivity sensor was calibrated. The conductivity sensor was connected to the WTW meter. The probe was inserted into the provided solution. The $<$ CAL $>$ key was pressed, then the $<$ RUN/ENTER $>$ key. Calibration was finished once AR stopped flashing. Finally the $\mathrm{pH}$ sensor was calibrated. The $\mathrm{pH}$ sensor was connected to the WTW meter and the $<\mathrm{CAL}>$ key was pressed. The $\mathrm{pH}$ probe was inserted into the $\mathrm{pH} 10$ buffer solution for 10 minutes. $<$ RUN/ENTER $>$ was then pressed to initiate a reading. After waiting for the reading to stabilize, the AR indicator stopped flashing. The probe was washed with distilled water. This step was repeated in $\mathrm{pH} 7$ buffer solution.

\subsubsection{Data Collection Methodology}

To measure DO, the DO sensor was connected to the WTW meter and placed directly into the stream (or into a bucket containing a water sample from the stream if restraints prevented the experimenters from getting to the water's edge). The $<$ AR $>$ key was pressed, followed by $<$ RUN/ENTER $>$ to initiate the reading. The probe was gently swished around within the water to ensure that a film did not develop on the probe and consequently alter the data. Once the 
reading stabilized, the AR indicator on the bottom right of the screen stopped flashing. Water temperature was measured at the same time as DO due to the relationship between water temperature and DO concentration. Measurements were taken three times to increase validity of data.

Next, conductivity was measured. This was done following the same steps as measuring DO. After the probe was plugged into the WTW meter, The $<$ AR $>$ key was pressed, followed by $<$ RUN/ENTER $>$ to initiate the reading. Once the reading was established, the AR indicator on the bottom right of the screen stopped flashing. Readings were taken three times to decrease the uncertainty of data collected.

Finally, pH was measured, again following the same procedures as DO concentration and conductivity. Once the $\mathrm{pH}$ probe cap was removed and the probe plugged into the WTW meter, the $<\mathrm{AR}>$ key was pressed, followed by $<$ RUN/ENTER $>$ to initiate the reading. Once the reading stabilized, the AR indicator on the bottom right of the screen stopped flashing. Three measurements were taken in order to decrease uncertainty.

\subsubsection{Colilert Testing}

In order to gain a comprehensive understanding of Boones Run water quality, coliform and E. coli testing was incorporated into the testing procedures in January 2019. During site visits, samples of approximately $100 \mathrm{~mL}$ of water were collected at each testing site in sterile containers and kept on ice to preserve each sample. Once back in the ISAT environmental lab, a Colilert reagent packet was added to each water sample. The water samples were mixed until substrate dissolution. Next, one sample was poured into the colilert Quanti-Tray, placed into a rubber insert, and pressed into a tray sealer. The necessary parts can be observed in figure 17. After the process was repeated for all samples, the samples were incubated at $35^{\circ} \mathrm{C}$ for 24 hours. After the incubation period had passed, readings were taken using the Most Probable Number (MPN) table [18]. 


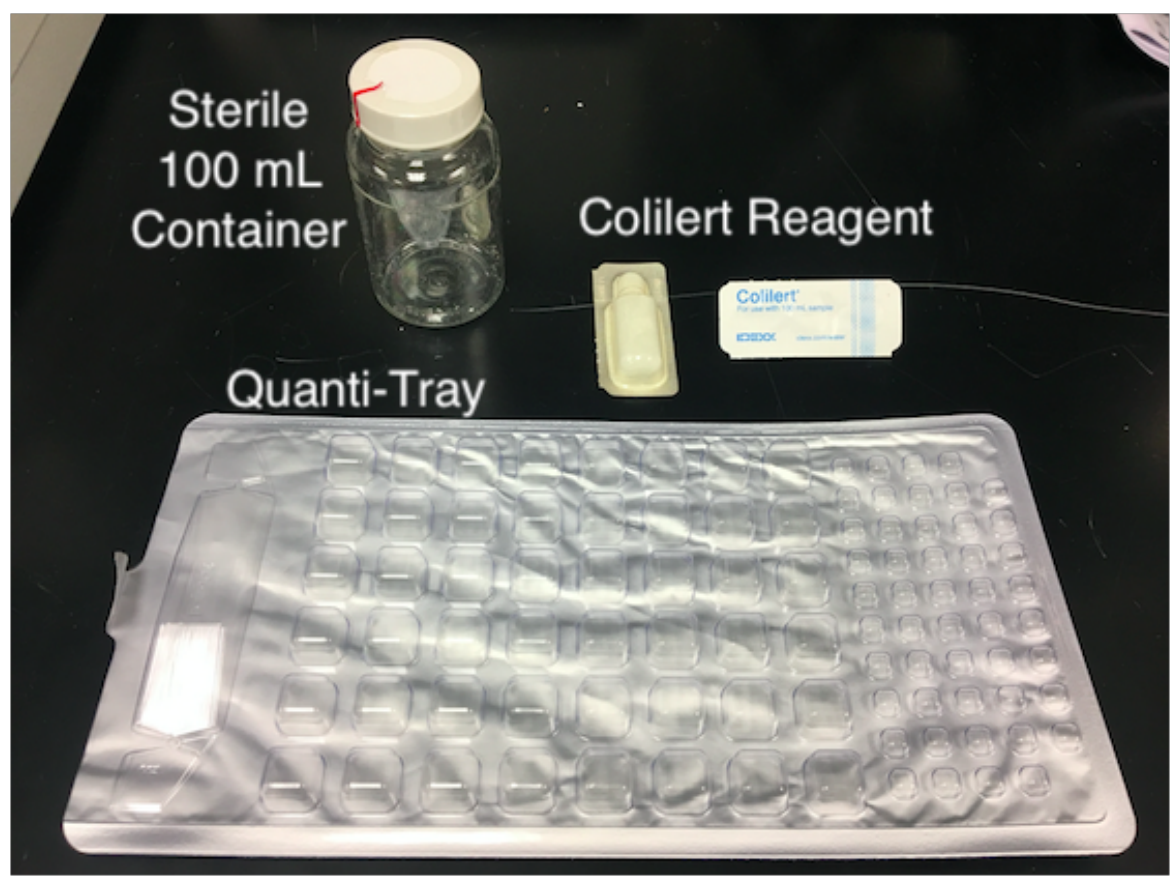

Figure 17: Materials used to complete a Colilert coliform and E. coli test.

\subsubsection{Water Quality Results}

Prior to the implementation of water quality sensors within Boones Run, experimenters monitored water quality manually via WTW meters. Experimenters recorded $\mathrm{pH}$, conductivity, $\mathrm{DO}$ concentration, and water temperature at four sites within Boones Run from the time period of September 2018 to March 2019 .

Table 2 displays the average DO concentration in miligrams per liter $(\mathrm{mg} / \mathrm{L})$ at each site and associated standard deviation. Site 2 is downstream of Site 1, Site 3 is downstream of Site 2, and Site 5 is downstream of Site 3. 


\begin{tabular}{cccc}
\hline Site & Sample Size (n) & $\begin{array}{c}\text { Average Dissolved Oxygen } \\
\text { Concentration }(\mathbf{m g} / \mathbf{L})\end{array}$ & Standard Deviation $(\mathbf{m g} / \mathbf{L})$ \\
\hline 1 & 8 & 11.46 & 1.42 \\
2 & 8 & 11.32 & 1.76 \\
3 & 8 & 11.10 & 2.05 \\
5 & 8 & 11.00 & 2.36 \\
\hline
\end{tabular}

Table 2: The average DO concentration and standard deviation $(\mathrm{mg} / \mathrm{L})$ at Sites $1,2,3$, and 5 .

Figure 18 displays the average DO concentration in $\mathrm{mg} / \mathrm{L}$ at Sites $1,2,3$, and 5. The horizontal line at $6 \mathrm{mg} / \mathrm{L}$ indicates the minimum DO concentration that can sustain populations of brook trout. The ' $x$ ' within the center of each box and whisker plot represents the mean for DO concentrations over time for each site. The black horizontal line in the middle of each 'box' represents the median DO concentration over time at each site. The upper perimeter line of the box, also known as the upper quartile, represents the number at which $25 \%$ of data values are greater than this point, and $75 \%$ of data values are below this point. The line above the upper quartile, also known as the upper extreme, represents the maximum value of the data set. The lower perimeter line of the box (the lower quartile), represents the number at which $75 \%$ of data values are greater than this point, and $25 \%$ of data values are below this point. The line below the lower quartile, the lower extreme, represents the minimum value of the data set. 


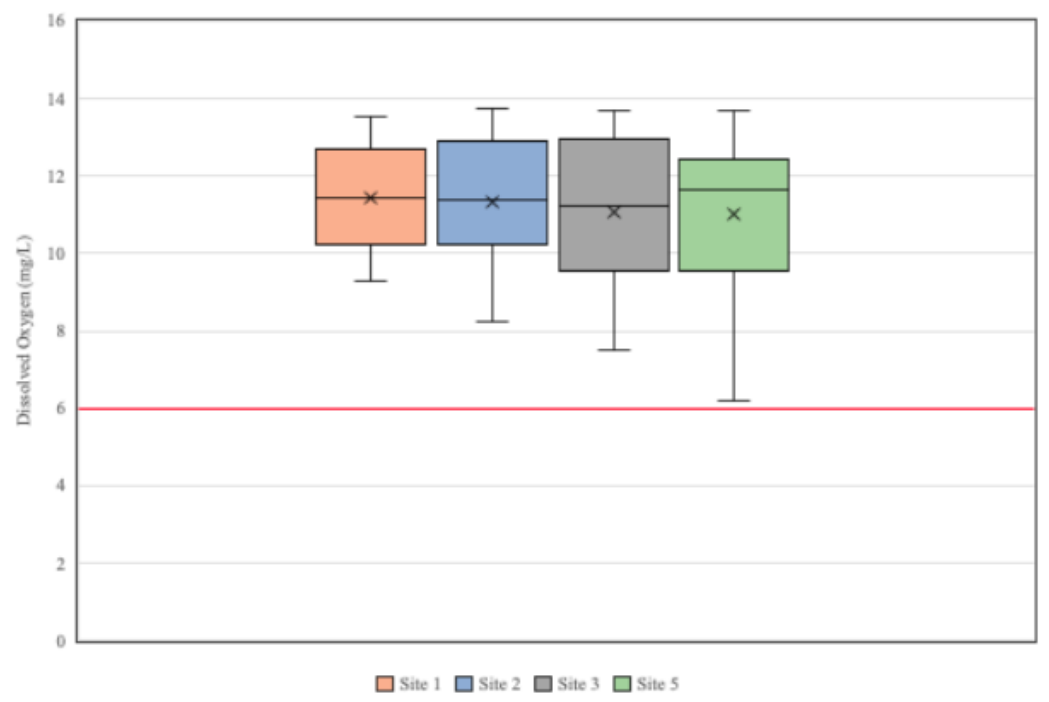

Figure 18: Average DO concentration $(\mathrm{mg} / \mathrm{L})$ at Sites $1,2,3$, and 5. The red horizontal line at $6.0 \mathrm{mg} / \mathrm{L}$ indicates that water with DO concentrations less than $6.0 \mathrm{mg} / \mathrm{L}$ will not sustain brook trout.

Table 3 displays the average DO percent saturation at each site and associated standard deviation.

\begin{tabular}{lccc}
\hline Site & $\begin{array}{c}\text { Sample } \\
\text { Size (n) }\end{array}$ & $\begin{array}{c}\text { Average Dissolved Oxygen } \\
\text { Percent Saturation }\end{array}$ & $\begin{array}{c}\text { Standard } \\
\text { Deviation }\end{array}$ \\
\hline 1 & 8 & 97.21 & 5.93 \\
2 & 8 & 95.44 & 6.54 \\
3 & 8 & 93.55 & 10.32 \\
5 & 8 & 91.88 & 13.52 \\
\hline
\end{tabular}

Table 3: The average DO percent saturation and standard deviation at Sites 1, 2,3 , and 5 .

Figure 19 displays the average DO percent saturation at Sites 1, 2, 3, and 5 . While DO concentration varies depending upon temperature, percent saturation 
indicates the percentage of oxygen dissolved within the water relative to saturation point (the point at which DO and atmospheric oxygen are in equilibrium). The point below the lower quartile at Site 3 represents an outlier within the data set $(70.59 \%)$. The point below the lower quartile at Site 5 also represents an outlier within the data set $(58.88 \%)$.

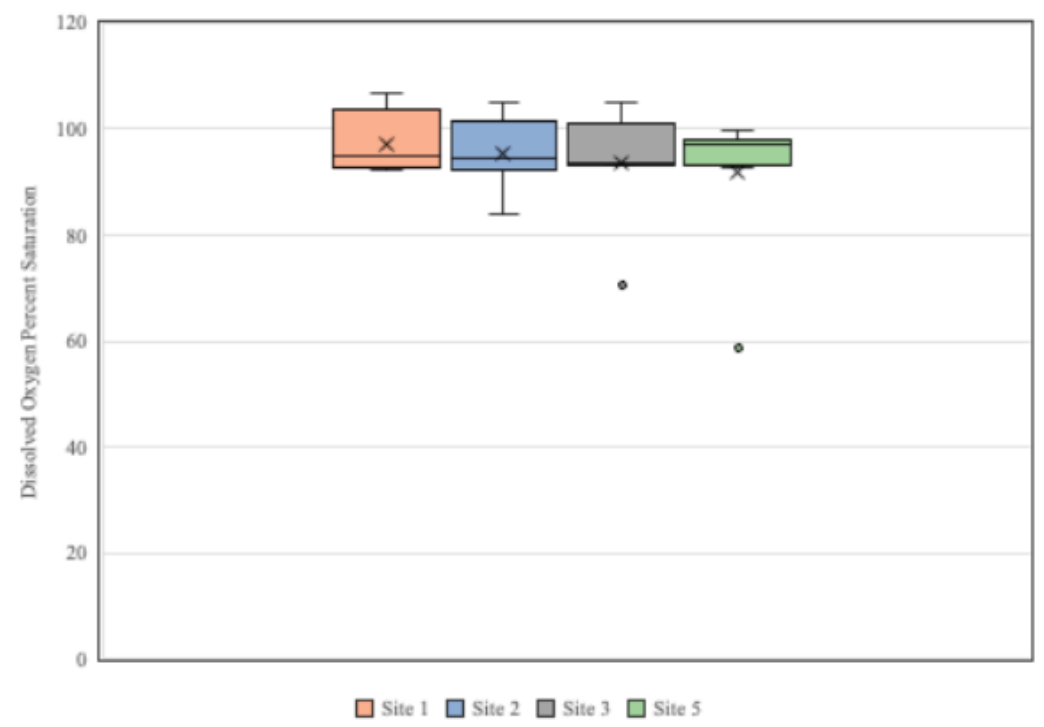

Figure 19: Average DO percent saturation from Sites 1, 2, 3, and 5.

Table 4 displays the average water temperature $\left({ }^{\circ} \mathrm{C}\right)$ at each site and associated standard deviation. 


\begin{tabular}{cccc}
\hline Site & Sample Size $(\mathrm{n})$ & Average Temperature $\left({ }^{\circ} \mathrm{C}\right)$ & Standard Deviation $\left({ }^{\circ} \mathrm{C}\right)$ \\
\hline 1 & 8 & 8.56 & \\
\hline & & & 4.30 \\
3 & 8 & 8.44 & 4.36 \\
& & & 4.34 \\
5 & 8 & 8.51 & 4.95 \\
\hline
\end{tabular}

Table 4: The average change in water temperature and standard deviation $\left({ }^{\circ} \mathrm{C}\right)$ at Sites 1, 2, 3, and 5 .

Figure 20 displays the trend in water temperature $\left({ }^{\circ} \mathrm{C}\right)$ at Sites $1,2,3$, and 5 over time.

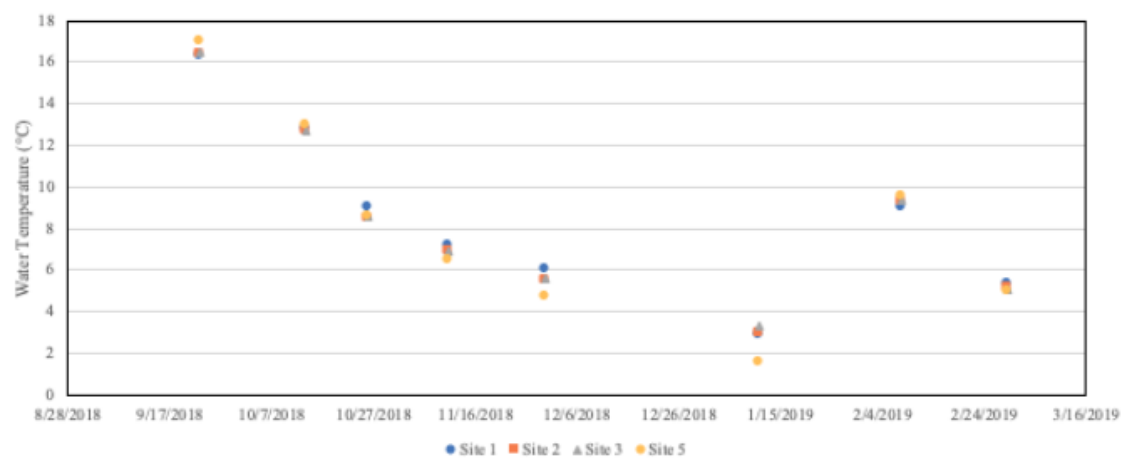

Figure 20: Average water temperature $\left({ }^{\circ} \mathrm{C}\right)$ over time at Sites 1, 2, 3, and 5.

Figure 21 displays the average air temperature $\left({ }^{\circ} \mathrm{C}\right)$ over time at Sites $1,2,3$, and 5 . 


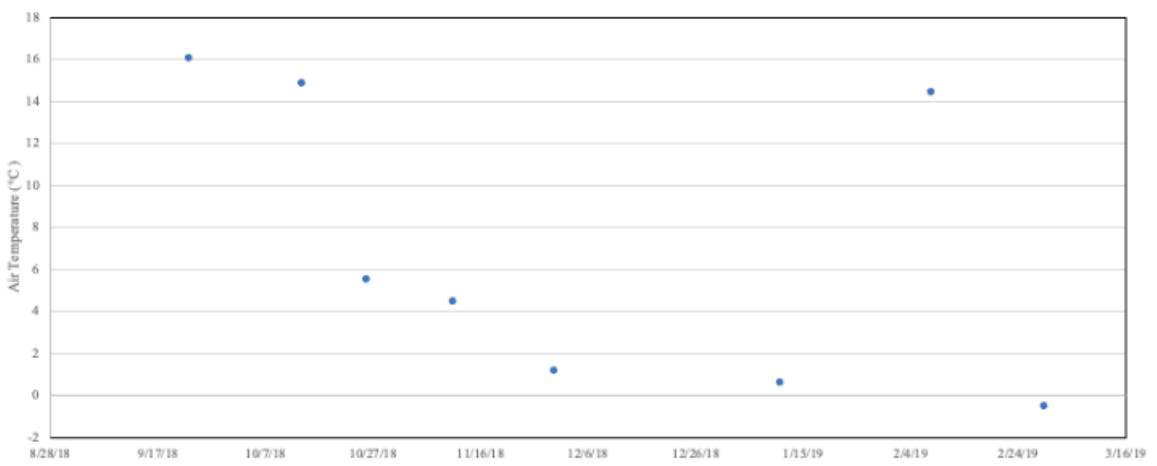

Figure 21: Air temperature $\left({ }^{\circ} \mathrm{C}\right)$ over time.

Table 5 displays the average conductivity $(\mu \mathrm{S} / \mathrm{cm})$ at each site and associated standard deviation.

\begin{tabular}{cccc}
\hline Site & Sample Size $(\mathrm{n})$ & $\begin{array}{c}\text { Average Conductivity } \\
(\mu \mathrm{S} / \mathrm{cm})\end{array}$ & Standard Deviation $(\mu \mathrm{S} / \mathrm{cm})$ \\
\hline 1 & 8 & 35.42 & 5.60 \\
2 & 8 & 42.79 & 5.50 \\
3 & 8 & 47.96 & 5.28 \\
5 & 8 & 77.50 & 7.60 \\
\hline
\end{tabular}

Table 5: The average conductivity and standard deviation $(\mu \mathrm{S} / \mathrm{cm})$ at Sites 1 , 2,3 , and 5 .

Figure 22 displays the average conductivity at Sites 1, 2, 3, and 5 . 


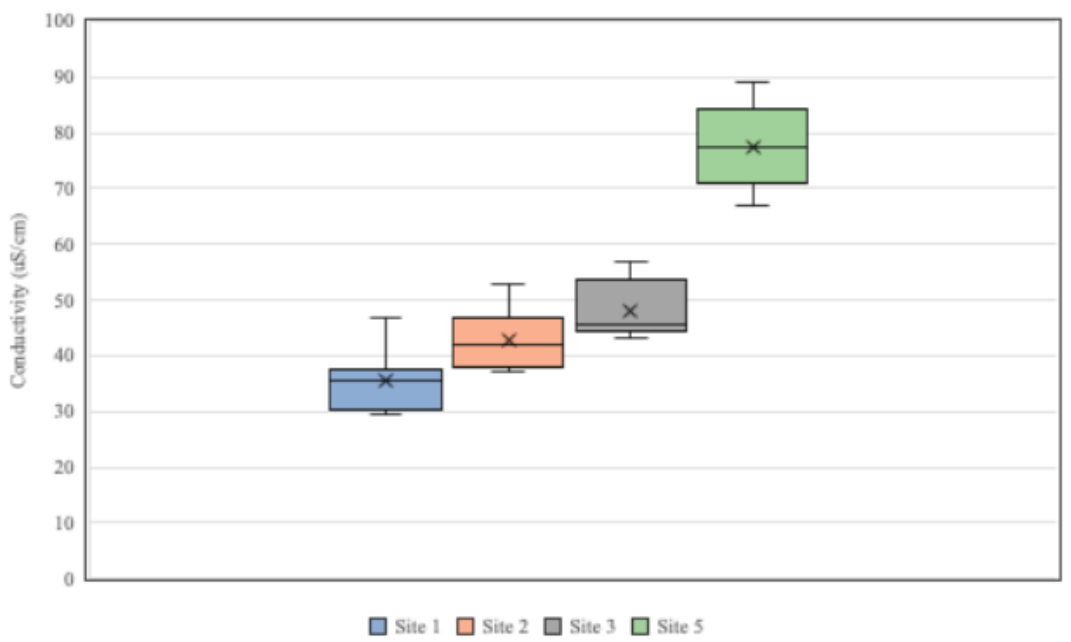

Figure 22: Average conductivity $(\mu \mathrm{S} / \mathrm{cm})$ at Sites $1,2,3$, and 5 .

Table 6 displays the average $\mathrm{pH}$ at each site and associated standard deviation.

\begin{tabular}{cccc}
\hline Site & Sample Size (n) & Average pH & Standard Deviation \\
\hline 1 & 8 & 7.43 & 0.35 \\
2 & 8 & 7.28 & 0.33 \\
3 & 8 & 7.23 & 0.38 \\
5 & 8 & 7.48 & 0.35 \\
\hline
\end{tabular}

Table 6: The average $\mathrm{pH}$ and standard deviation at Sites 1, 2, 3, and 5 .

Figure 23 displays the average $\mathrm{pH}$ at Sites $1,2,3$, and 5. The horizontal lines at $\mathrm{pH} 6$ and 9 indicates the minimum and maximum $\mathrm{pH}$ values (respectively) that can sustain populations of brook trout. 


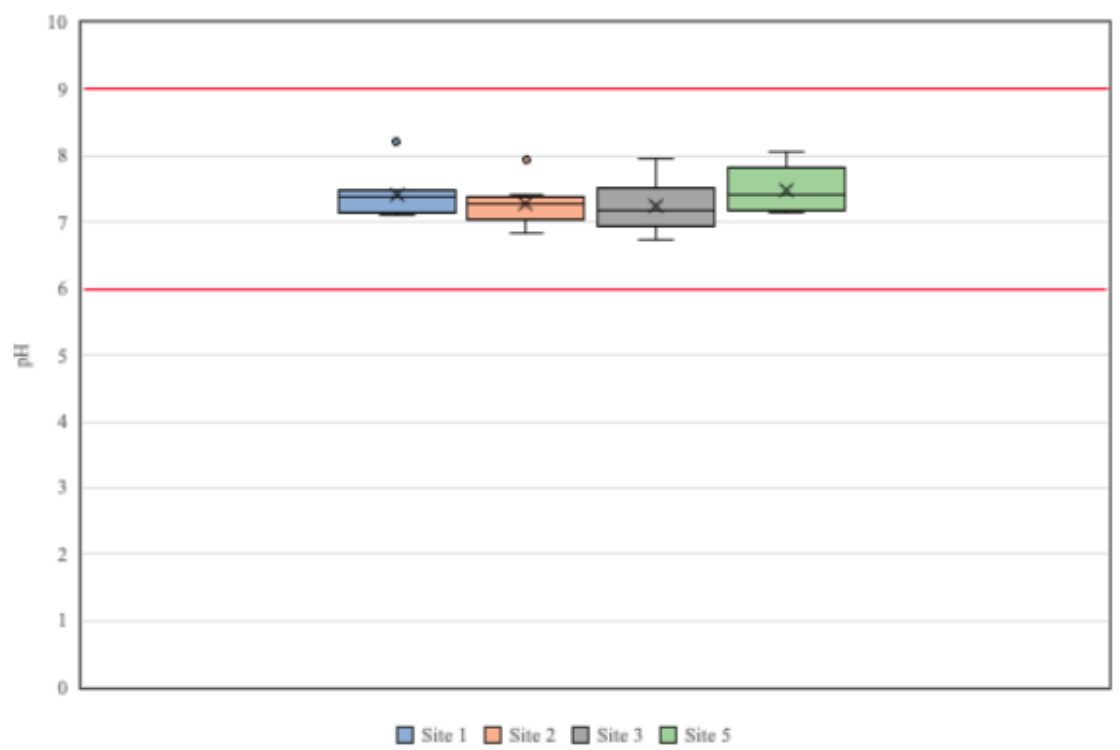

Figure 23: Average stream pH over time at Sites 1, 2, 3, and 5. The red horizontal line at $\mathrm{pH} 9$ represents the upper bound of tolerable conditions for brook trout, while the red horizontal line at $\mathrm{pH} 6$ represents the lower bound of tolerable conditions [23].

\subsubsection{Colilert Testing Results}

Experimenters began conducting Colilert testing in January 2019. This additional test helped to create a more holistic understanding of water quality within Boones Run. As Boones Run travels through residential and agricultural land, it was expected that concentrations of coliform and E.coli would increase the further downstream testing was conducted. Although the sources of E.coli contamination were not confirmed, leaking septic systems and/or agricultural runoff containing fecal matter may be the culprit.

Table 7 displays the coliform and E.coli concentrations measured at each site across the three testing days. The geometric mean was calculated to help rectify outlying data points likely caused by a change in weather. Due to a testing error, several cells were punctured while sealing the Quanti-Tray. Due to this error, the average of the inferred maximum and the inferred minimum were used as the microbial concentration present in the damaged cells could not be definitively determined. Inferred minimum refers to the assumption that each of the compromised cells was negative for coliform, while the inferred maximum refers to the assumption that each of the compromised cell was positive for coliform. If E.coli concentrations were less than one, the geometric mean was calculated using 1 , as the mean can not be calculated without a definitive number. 


\begin{tabular}{|c|c|c|c|c|}
\hline \multicolumn{5}{|c|}{ Coliform $(\mathrm{cfu} / \mathbf{1 0 0 m L})$} \\
\hline & 1/11/19 & $2 / 8 / 19$ & $3 / 1 / 19$ & Geometric Mean \\
\hline Site 1 & 19.1 & 46 & 727 & 86.12 \\
\hline Site 2 & 27.4 & 39.9 & 461.1 & 79.59 \\
\hline Site 3 & 61.3 & 64 & 686.7 & 139.15 \\
\hline Site 5 & 129.5 & 2419.6 & 804.25 & 631.64 \\
\hline \multicolumn{5}{|c|}{ Escherichia coli $(\mathrm{cfu} / 100 \mathrm{~mL})$} \\
\hline & 1/11/19 & $2 / 8 / 19$ & $3 / 1 / 19$ & Geometric Mean \\
\hline Site 1 & $<1$ & 1 & $<1$ & 1 \\
\hline Site 2 & $<1$ & 1 & 1 & 1 \\
\hline Site 3 & 4 & 12.2 & 3 & 5.27 \\
\hline Site 5 & 31.8 & 2419.6 & 46.25 & 152.67 \\
\hline
\end{tabular}

Table 7: Coliform and E.coli concentrations measured using a MPN Chart.

Figure 24 shows a bar chart of the geometric means of coliform and E.coli concentrations at each site across all three sampling dates. The Site 5 samples on $3 / 1 / 19$ were compromised when several cells were punctured during the tray sealing process. The average of the inferred maximum and the inferred minimum were used as the cells were compromised.

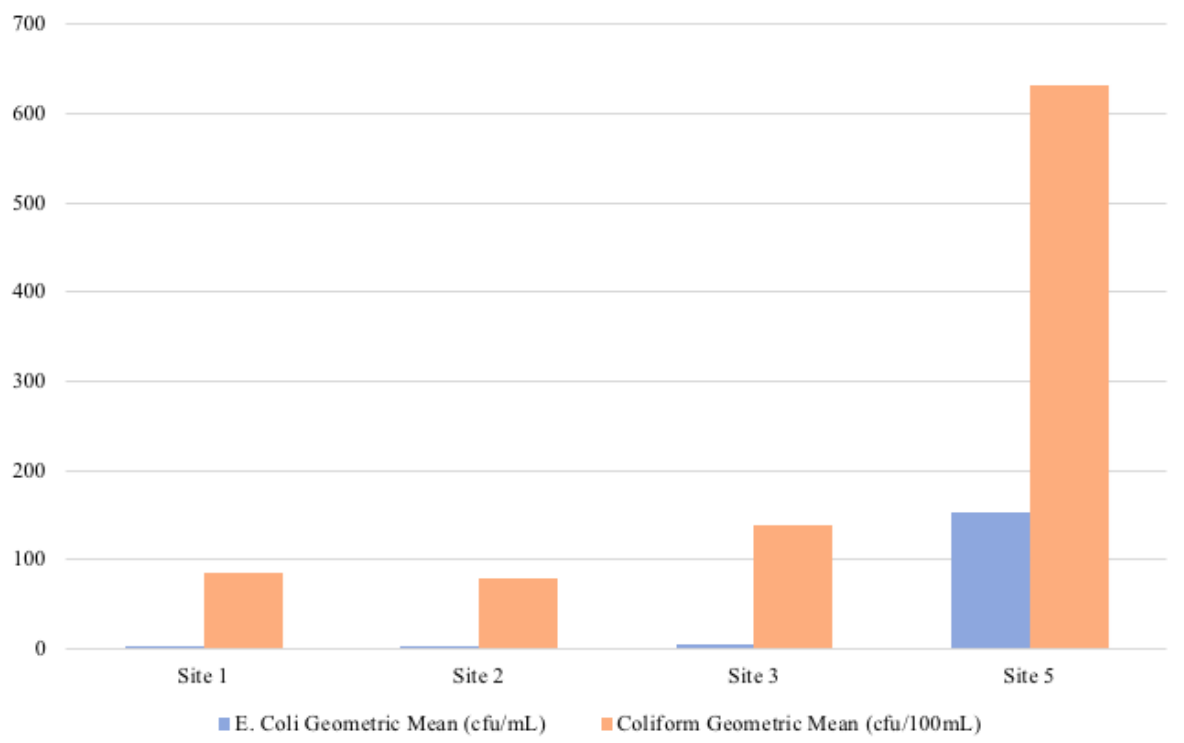

Figure 24: Bar chart illustrating the geometric means of coliform and E.coli concentrations at each site. 


\subsection{Discussion}

In an effort to assess the water quality of Boones Run, the authors of this report tested water quality at Sites $1,2,3$, and 5 on eight separate occasions between the dates of September 23, 2018 and March 1, 2019. Data regarding DO concentration, DO percent saturation, water temperature, conductivity, and $\mathrm{pH}$ were amassed. Additionally, 3 water samples were collected between January and March in order to conduct Colilert testing. The Colilert test determined the total coliform and E.coli in the water samples.

DO concentrations were collected to assess the suitability of Boones Run for brook trout populations. Natural trout waters (class VI) must have a minimum DO concentration of $6 \mathrm{mg} / \mathrm{L}$ to sustain trout, but average $7 \mathrm{mg} / \mathrm{L} /$ day [23]. As evidenced by Figure 18, average DO concentrations at Sites 1, 2, 3, and 5 were consistently greater than $6 \mathrm{mg} / \mathrm{L}$. The lowest average DO concentration was $11.00 \mathrm{mg} / \mathrm{L}$ at Site 5, while the highest average concentration was 11.46 at Site 1. Notably, Site 1 is within the George Washington National Forest, while Site 5 intersects a major roadway. DO percent saturation was derived from concentration data to represent DO levels as a percentage. When represented as a concentration, DO is contingent upon water temperature data. DO percent saturation illustrates the data on scale independent of explicitly referencing water temperature. Average dissolved oxygen percent saturation aligned with the aforementioned concentration data: the highest percentage of DO was at Site 1, and the lowest at Site 5. Further investigations must be conducted to determine causative factors.

Water temperature directly influences DO concentration: as temperatures decrease, DO levels increase. DO levels and water temperatures were measured simultaneously to accurately analyze the relationship between DO levels and water temperature. As evidenced by Figure 20, water temperature at Sites 1, 2, 3, and 5 collectively decreased between September 23, 2018 and January 1, 2019. The decrease in water temperature corresponded with a decrease in air temperature, as evidenced by Figure 21. Water temperature increased on February 8, 2019 before decreasing again on March 1, 2019. The lowest air temperature was $-0.56{ }^{\circ} \mathrm{C}$, while the highest air temperature was $16.0{ }^{\circ} \mathrm{C}$.

In addition to DO concentration, other variables, including the $\mathrm{pH}$ of water, impact the ability of a stream to sustain aquatic life. Natural trout waters between a pH range of 6 and 7 sustain trout [23]. As evidenced by Figure 23, the average $\mathrm{pH}$ at Sites 1, 2, 3, and 5 did not exceed a $\mathrm{pH}$ of 9 and consistently remained above a $\mathrm{pH}$ of 6 . The highest average $\mathrm{pH}(7.48)$ was recorded at Site 5 , while the lowest average $\mathrm{pH}$ (7.23) was measured at Site 3. Further investigations must be conducted to determine causative factors between differences in $\mathrm{pH}$.

The last water quality metric that the authors of this report assessed was conductivity of the water. Conductivity refers to the concentration of ions capable 
of conducting electricity within the water. As evidenced by Figure 22, conductivity varied between Sites 1, 2, 3, and 5 . The lowest average reported concentration of conductivity was $35.42 \mu \mathrm{S} / \mathrm{cm}$ at Site 1 , while the highest average conductivity was 77.50 at Site 5 . Further investigations must be conducted to determine causative factors regarding the downstream difference in conductivity, although it is likely due to the land use adjacent to Site 5 versus Site 1 . As previously mentioned, Site 1 was within George Washington National Forest, while Site 5 intersected a major roadway and was in close proximity to several residences. Speculations regarding high conductivity include runoff from the road (such as salt used to prevent ice accumulation) and faulty sewage systems.

Total coliform bacteria are a collection of microorganisms found in the intestines of both warm and cold blooded animals and also free floating in environmental reservoirs. An outlying organism within total coliform is E.coli, which are distinguished by their ability to grow at elevated temperatures and are specifically associated with the fecal material of warm-blooded animals [24]. As a result, they are an indicator that there is a potential sewage system failure or agricultural runoff. As observed in the total coliform and E.coli data, Site 5 exhibited high levels of E.coli suggesting that fecal matter had entered Boones Run. Although E.coli in small concentrations does not have the potential to cause harm, presence of E.coli suggests that other pathogenic organisms have entered the waterway, which could cause infection or bodily harm. Additionally, other tributaries that join Boones Run between Site 3 and 5 likely possess high levels of total coliform, contributing to the high level of total coliform and E.coli at Site 5. Site 1 is in the George Washington National Forest, and as there are not high levels of total coliform found free floating in waterways, it is expected there would be low concentrations of microorganisms detected. More data regarding the source of E.coli would be needed to determine the possible sources of total coliforms in Boones Run.

Stakeholders with a vested interest in the aforementioned results include residents of the watershed impacted by the DuPont mercury contamination, as they fish, swim, and rely upon water sourced from the watershed. Residents may experience health impacts from consumption and/or exposure to the water. Further, fishermen and other individuals involved in industries focused upon sourcing fish and other organisms from contaminated waters may find their livelihoods threatened by fish consumption bans within the area. The area is highly sought out by residents and tourists alike for the vast array of recreational activities that the area offers. This leaves visitors with potential risk of mercury exposure which could lead to life threatening impacts. Additionally, there are economic impacts to the surrounding community due to a decrease in recreational use as a result of perceived risk. For example, fish anglers may avoid fishing within contaminated rivers, even if they have no intention of consuming their catch. Research has found that individuals make choices based on their own personal risk assessment. Thus, fishers are likely to follow their perception of risks associated with fishing in waters with advisories and are more likely to 
choose sites that they believe do not pose a risk to their well-being, despite the fact that fishing for purely recreational purposes poses no threat to fishers [25].

DuPont, the company responsible for the mercury contamination of the river, is also a stakeholder relevant to this problem. The money owed as a result of a settlement when the state of Virginia sued DuPont, was parsed into different projects in the surrounding area. DuPont is financially liable for managing the mercury contamination, and funding environmental remediation projects. DuPont contaminated the river without consideration that their actions impact the health and well-being of residents dependent upon this water. What obligation do corporations, such as DuPont, have to protect the quality of water? DuPont, and all of the other entities that contaminated the South Fork of the South River have a moral responsibility to the community and the environment that their company impacts. DuPont was heavily criticized for the damages they caused to the ecosystem, and paid the price with a $\$ 42$ million dollar settlement to help counteract the damages. Other companies did not demonstrate this social responsibility. Further, recipients of the litigation fund, such as the Virginia Department of Forestry, are invested in the issue, as they are tasked with engaging in restoration activities following awarding of the money.

Additional stakeholders relevant to this issue include regulatory organizations involved in monitoring and maintaining water quality, such as the U.S. EPA and VADEQ. These agencies enforce water quality standards and hold localities accountable for the set of applicable standards. Additionally, the United States Fish and Wildlife Service monitor the potential impacts on terrestrial and aquatic organisms, and have a stake in how contamination and restoration efforts may impact wildlife. 


\section{The Drone}

\subsection{Literature Review}

Wireless sensor networks (WSNs), in conjunction with unmanned aerial vehicles (UAVs), are often implemented in research settings to expedite the process of data collection and to increase the breadth of data that may be collected over a geographical area. Traditionally, marine ecosystemic health have been monitored via satellite imagery, submerged aquatic sensor devices, and devices placed upon buoys. Extracting data from buoys often involves transmitting data to pre-existing ground stations, wireless range which may be limited to 100 meters or less. Moreover, if ground station infrastructure does not exist, researchers may be required to travel to buoys to collect data or the buoy itself. Costs associated with maintenance, paying for labor, and monthly fees limits the extent to which data may be collected.

Trasviña-Moreno et. al of the Aragon Institute of Engineering Research constructed a WSN to monitor a marine-coastal environment within Mexico, data which was collected with a UAV [26]. Figure 25 illustrates the architectural schematic of a WSN constructed for the purpose of the study. Sensor devices placed upon buoys collected data on environmental metrics, such as water temperature. Data was transmitted to a fixed-wing drone, and then to a ground station.

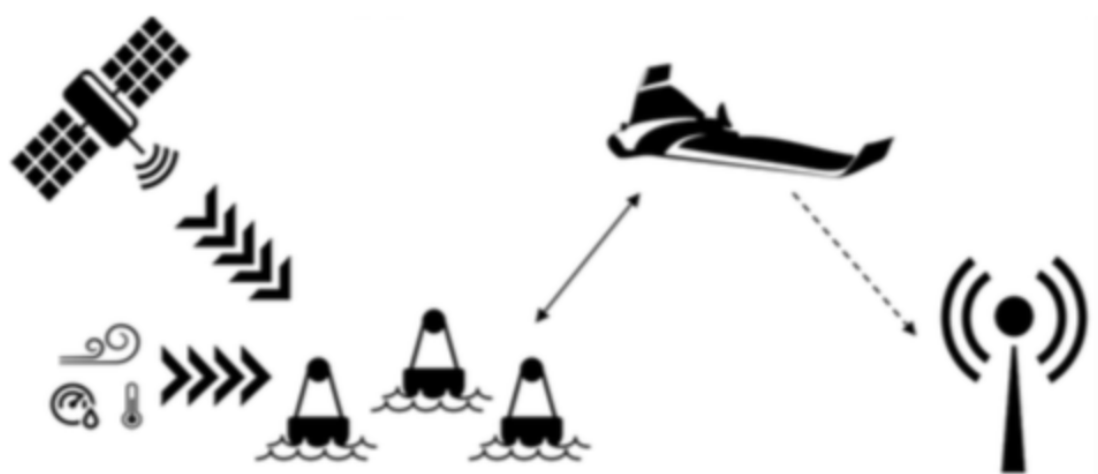

Figure 25: The WSN constructed to monitor a marine-coastal environment, components of which included environmental sensors placed upon buoys, a fixedwing drone to collect the data, and a ground-station to which data was transmitted [26].

Figure 26 illustrates the functional purpose of each component within the network, beginning with sensor nodes placed upon the buoys. The central node 
placed upon the UAV retrieved data from the sensor nodes and contained a coding system to parse the data. A control node placed within a ground-station logged data.

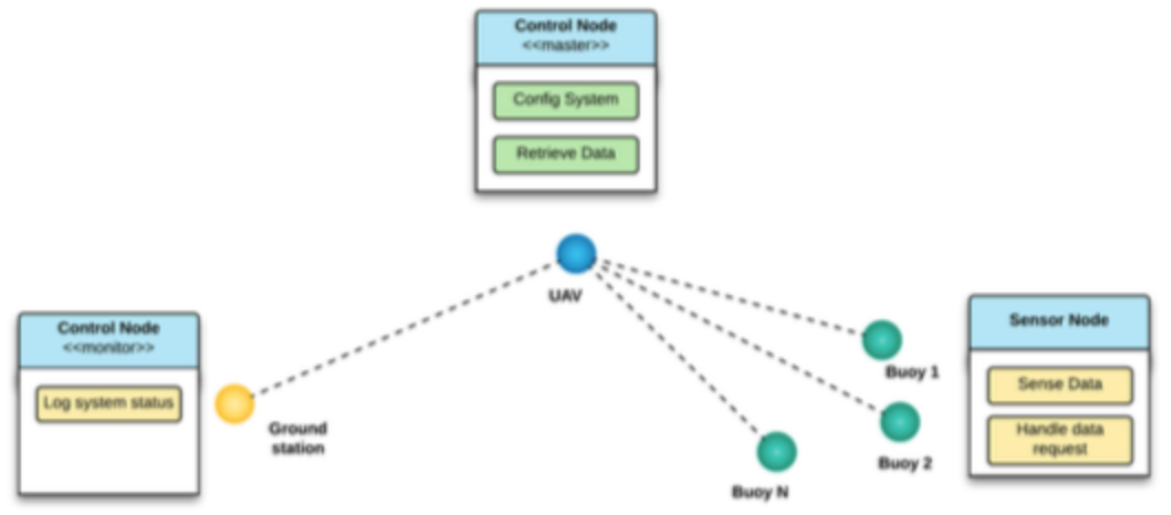

Figure 26: Schematic representing data flow from the sensor nodes upon buoys to the control node located on the UAV and finally to a control node at a ground station [26].

Following buoy deployment within the testing location, the UAV with the control node was launched on a pre-set path, network connectivity monitored with a computer on the ground. Throughout the process of data extraction, researchers monitored data relating to wind speed, air temperature, water temperature, relative humidity, and atmospheric pressure in real-time. At the conclusion of the study, the use of UAVs to remotely extract environmental data was validated. Although testing was contained within a marine environment, the proofof-concept may be applied to a variety of scenarios, including collecting data from inaccessible, dangerous areas [26].

The communication between the sensor nodes and the UAV was performed using LoRa technology. In our setup, we use WiFi communication as all the nodes are equipped with WiFi capability, and to prevent transmission delays [26].

WSNs and UAVs have also been used to monitor greenhouse gases. As explained by Malaver et. al, using WSNs and UAVs currently represent the best alternative to monitor large, remote, and difficult to access areas. Malaver et. al developed and explored the integration of a WSN with an UAV to monitor nanostructured metal oxide (MOX) as well as concentrations of $\mathrm{CH}_{4}$ and $\mathrm{CO}_{2}$ in the atmosphere. Data collected during experimentation was transmitted in real time to a central node for analysis and 3D mapping of the gases in concern. Traditional, large scale monitoring of these greenhouse gases produced by industry and agriculture is a demanding task that requires extensive periods 
of observation, long term stability, computational resources, and places high demands on energy [27].

The WSN constructed in the experiment conducted by Malaver et. al, consisted of sensor nodes with microprocessors that had networking capabilities. The nodes in charge of sensing the gases in the atmosphere were powered by solar panels. Data collected from the sensor nodes was stored and displayed in realtime on webpages. Refer to Figure 27 for how the WSN, UAV, and the data management platform webpage were all integrated together [27].

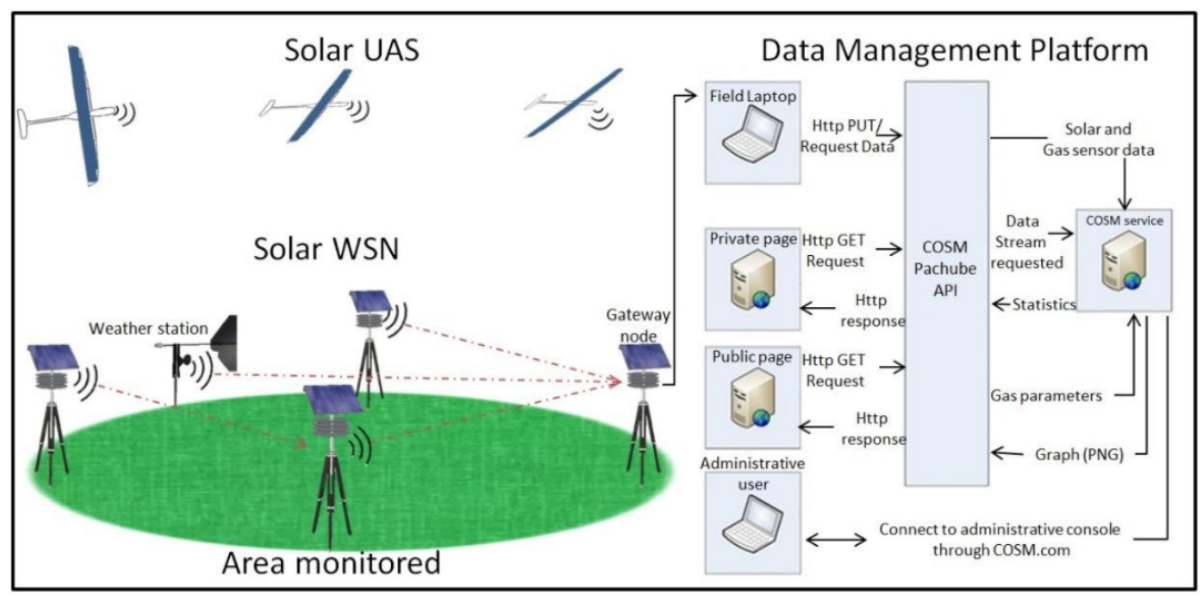

Figure 27: Illustrated the design of a solar powered WSN and a UAV integrated into a data management platform for continuous monitoring of pollutant gases used by Malaver et. al [27].

The first field test conducted by Malaver et. al involved two nodes used to monitor $\mathrm{CO}_{2}$ at ground level and the UAV flying above the nodes to collect the data. Another test integrated a base node, one $\mathrm{CO}_{2}$ ground node, a weather station, and an UAV. The flight operation in this experiment lasted for 20 minutes and the base nodes were able to successfully connect to the UAV. Refer to Figure 28 for evidence of a 3D map constructed in Google Earth of the geo-location of where the nodes were placed that were used to collect data [27]. 


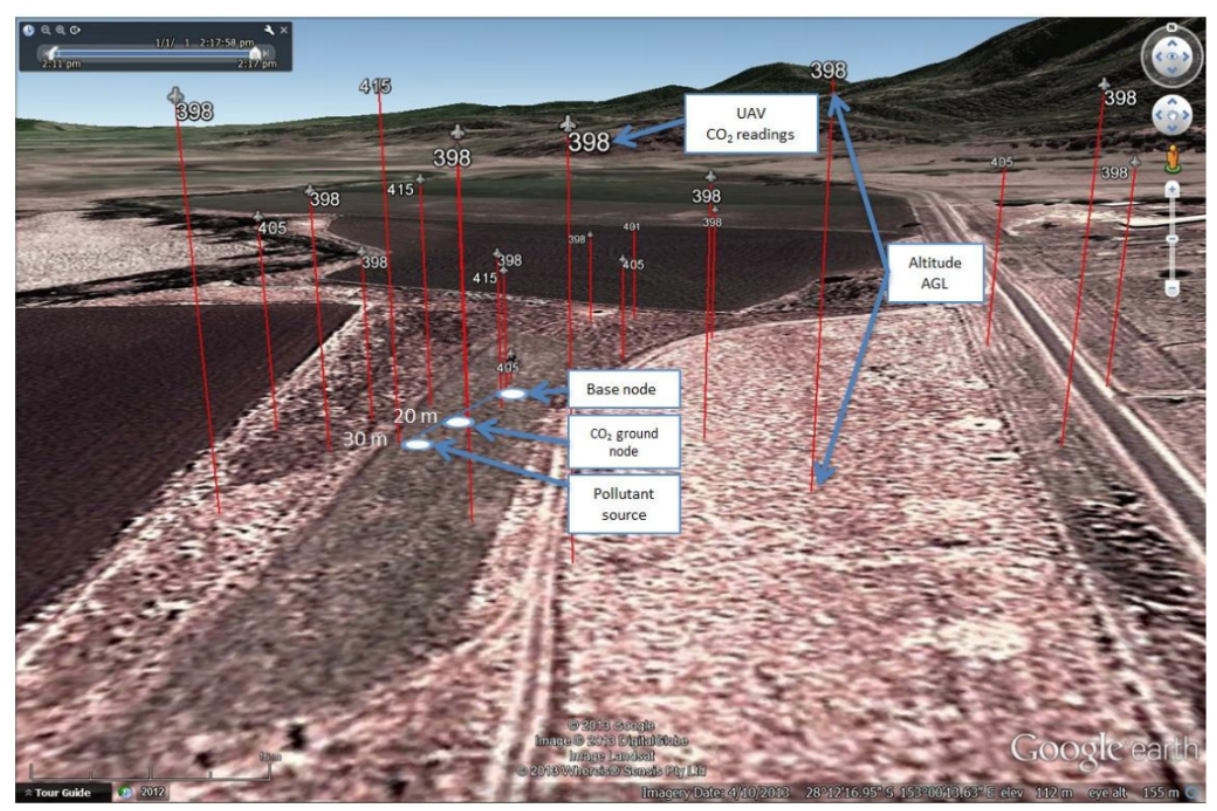

Figure 28: Geo-location of nodes the UAV collected data from Malaver et. al, 2015 [27].

Malaver et. al were able to prove that an UAV could be integrated with a WSN that monitored pollutant gases within the atmosphere. While the system constructed in this study was a prototype, it is important for the future of environmental monitoring. The concepts explored in this experiment will be applied to the data collection procedures used within this capstone. Many of the environmental sensors used within this capstone may be difficult to access. This study proves that using an UAV alleviates this issue and makes data collection possible even in areas that are challenging to access [27].

One advantage that the system described in this paper has over the one completed by Malaver et al. is that it is easy to deploy. The system described by Malaver et al. involved many different subsystems that required a larger scale integration of numerous different parts. The system described in this paper is a simplified environmental monitoring solution that also allows for continuous data collection. Additionally, the authors monitored atmospheric data and therefore placed less concern on waterproofing the system as we do in this project. 


\subsection{Background Information}

\subsubsection{Unmanned Aerial Vehicles and their Use}

UAVs, more commonly referred to as drones, are powered aircraft that are operate either remotely or via a pre-programmed flight plan. UAVs offer a cheaper alternative to acquiring high resolution remote-sensing data with increased operational flexibility [28]. Historically, UAVs have been primarily used by the military. In recent years, UAVs have become more accessible to the public due to dramatic reduction in size, cost, and increased ease of use and functionality. As a result, new applications of UAVs have emerged, leading to novel prototypes and project applications. New civilian applications for UAvs include weather monitoring, forest fire detection, traffic control, emergency search and rescue, and communication relaying [29].

UAVs can be classified into two main categories: fixed wing (airplane) and rotary wing (helicopter). One of the more recent applications of fixed wing UAVs has been in monitoring and mapping the extent, biomass, and health of vegetation cover, including tidal wetlands, forests, and agricultural crops. New hyperspectral and multispectral imagers are being developed and incorporated into the construction of UAVs to capture better images of the observed area. Additionally, small UAVs have been used to generate high quality, time averaged images that provided detailed information on nearshore, including "sandbar morphology", and the dimensions of surf/swash zones [29]. Another study released used a UAV to study the gradual sinking of land as a result of underground coal mining that can alter the structure of the rock formations that support the land. The major advantage that rotary wing UAVs have over fixed wing UAVs is that they have the ability to hover over a target site, can descend on the site for closer inspection, and can easily change altitude to provide preferential imaging capabilities [29]. One historical use of rotary wing UAVs has been in coastal management. UAVs have been used to quantify the morphosedimentary changes of coastal fringe, including cross-shore and longshore sediment transport [29]. The wide range of uses of UAVs have the capability to fill in observation gaps in environmental remote sensing.

\subsubsection{Public Policy Surrounding Unmanned Aerial Vehicles}

Currently, the use of UAVS is governed by the Federal Aviation Administration (FAA). The FAA has implemented various operational limitations on flying UAVs. Some of these include that unmanned aircraft must weigh less than 55 pounds (25 kilograms) and that they must yield right of way to other aircraft. Additionally, the UAV must remain in visual line-of-sight (VLOS) of the remote pilot controlling the flight path of the UAV or it must remain in VLOS of the visual observer. Additionally, UAVs may not operate over any person not directly participating in the operation and not under a covered structure. The FAA also mandates that UAVs must only be operated during daylight hours, or civil twilight (30 minutes before official sunrise to 30 minutes after official sunset, 
local time) assuming that the UAV is equipped with sufficient anti-collision gear and lighting. UAVs can fly at a maximum groundspeed of 100 miles per hour and at a maximum altitude of 400 feet above ground level (AGL) or, if higher than 400 feet AGL, remain within 400 feet of a structure. When flying a UAV, the minimum weather visibility must be of three miles from where the UAV is being controlled. Additionally, no one person may act as a remote pilot in command or a visual observer for more than one UAV operation at one time. The FAA does allow for external load operations on UAVs if the object being carried by the unmanned aircraft is securely attached and does not adversely affect the flight characteristics or controllability of the aircraft [30].

Beyond operation limitations of operating UAVs, the FAA has also established restrictions on the remote pilot in command's certification and responsibilities. One of these established responsibilities is that a person operating a UAV must either hold a remote pilot airman certificate with a small UAV rating or be under the direct supervision of an individual who does hold a remote pilot certificate. In order to qualify for a remote pilot certificate, one must demonstrate aeronautical knowledge by passing an aeronautical knowledge test at an approved FAA testing center or by holding a Part 61 pilot certificate, completing flight review within the past 24 months, and by completing an online training course provided by the FAA on UAVs. Additionally, to qualify for a remote pilot certificate one must be vetted by the Transportation Security Administration (TSA) and be at least 16 years of age. Applicants will receive a temporary license after completion of the vetting process by the TSA. Finally, remote pilots must make the UAV available to the FAA for inspection or testing, report to the FAA of any operation that results in a serious injury or property damage of at least $\$ 500$, and conduct a pre-flight inspection to ensure that the UAV is in good condition for safe operation [30].

Ethical considerations involve the use of UAVs in a manner that may be harnessed to violate an individual's privacy. Homeowners and organizations may fear the placement of sensor nodes within their personal property, and the collection of data via UAV. If UAVs may be utilized to collect data from a distance, sensitive data regarding an individual or an organization may be at stake. For example, cameras may be placed throughout an individual's property, and visual footage pertaining to their activities may be gathered discreetly. The use of UAVs as an emerging technology has been prevalent in the past decade, and there is no sign of phasing them out in the near future. While UAVs are a relatively new technology, there has been detailed legislation, court cases, and proper use doctrines made public in which citizens should abide to. 


\subsection{Materials and Methods for Drone Assembly}

\subsubsection{Physical Assembly of Drone}

A DJI F450 quadcopter main frame UAV was chosen for use in this project. This UAV (which required assembly) was chosen as opposed to an out-of-thebox UAV because of the customization abilities. Out-of-the-box UAVs allow for minimum customization in comparison to the DJI F450. The ability to mount a Raspberry Pi 3 as well as a portable battery to power the device was an additional payload requirement for the UAV, which cannot be easily mounted onto most pre-assembled UAVs.

1: To assemble the the main frame of the DJI F450, the video titled "Assembling Frame of F450" was followed found at this link: [31].

2: To attach the remaining top piece of the UAV, the video titled "Naza-M Main Controller Assembly" was followed beginning at 2:13-2:34. This video can be found using the link included in Step 1.

3: After the UAV was calibrated, the landing legs were attached according to Figure 29 to the bottom piece of the UAV.

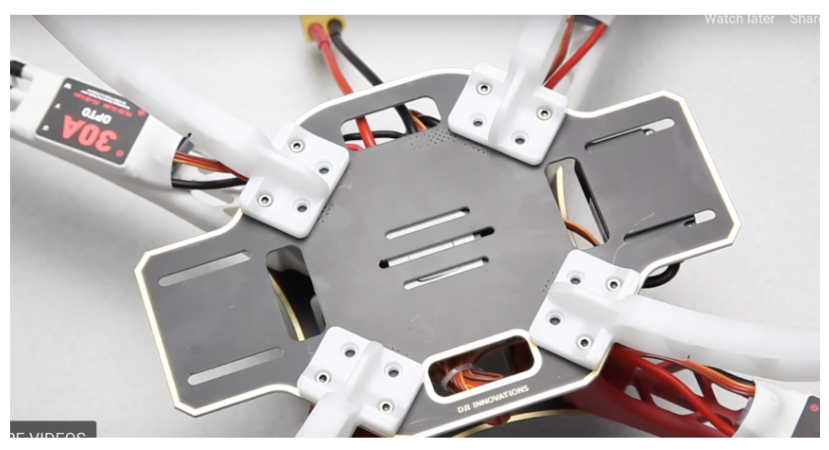

Figure 29: This figure illustrates how the landing legs were attached to the UAV.

4: The propellers were then attached to the motors. There were two propellers that matched the two counterclockwise moving motors and two propellers that matched the two clockwise moving motors. The motors should List $\mathrm{C}$ or CC in regards to the direction of the propellers it requires. The propellers were attached in the same way depicted in Figure 30. 


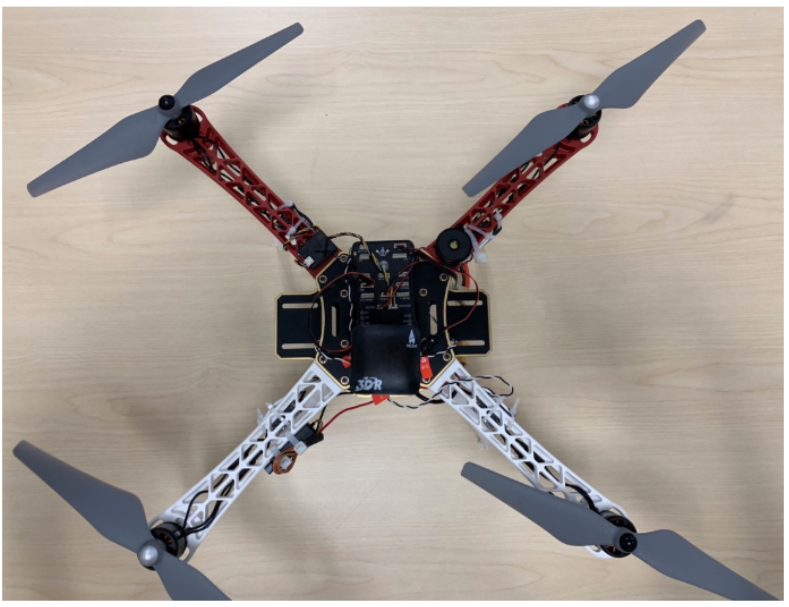

Figure 30: Correct propeller attachment on the drone. The red arms are the front of the UAV and the white arms are the back.

5: A Raspberry Pi 3, configured as an access point, was mounted upon the undercarriage of the UAV. Reference Figure 31 for a photograph of the RPI 3 mounted upon the UAV. 


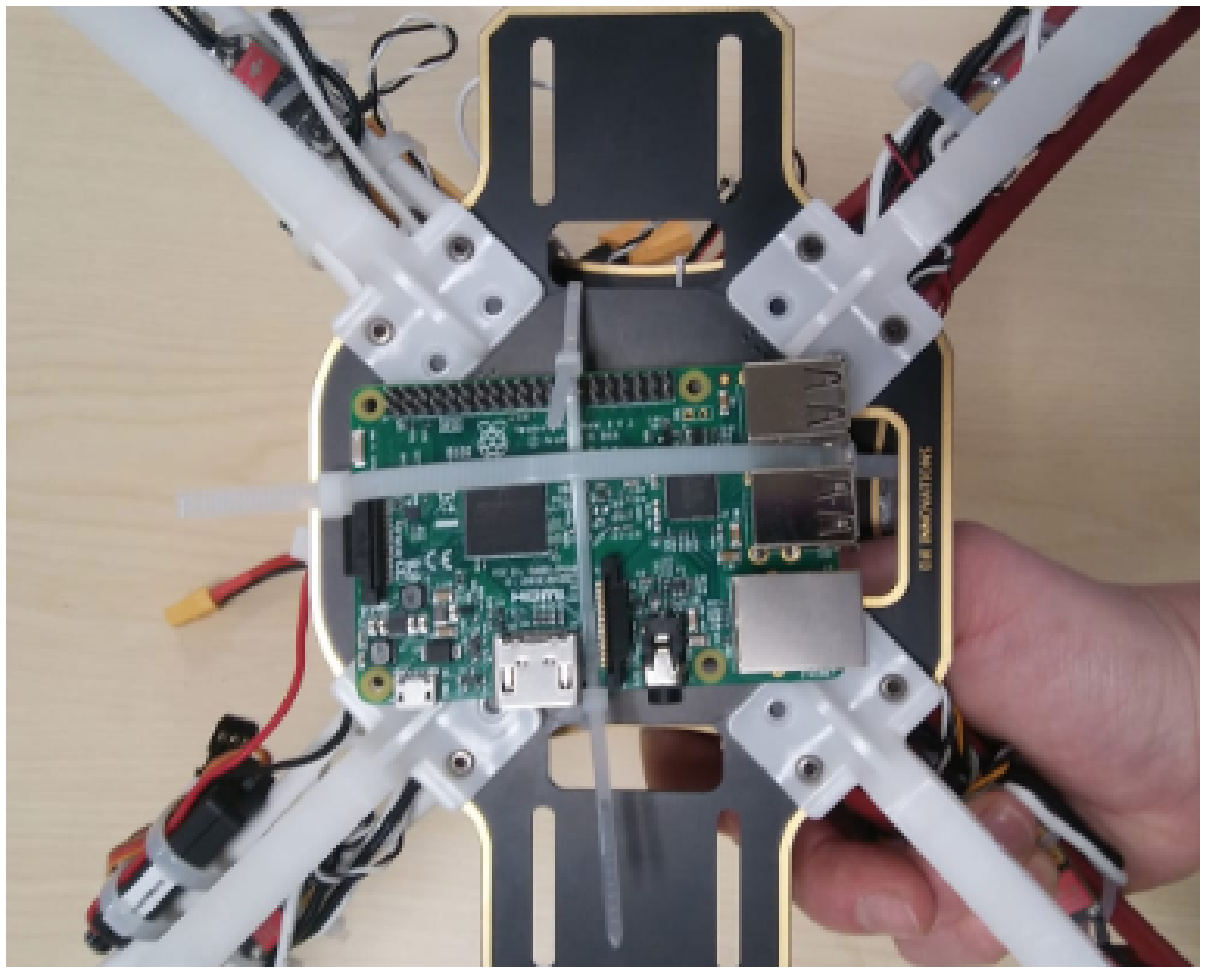

Figure 31: The Raspberry Pi 3 mounted to the undercarriage of the UAV.

6: In order to ensure the safety of the UAV, the propellers and landing legs were removed until the UAV had been paired, calibrated, and was ready for flight.

\subsubsection{Wiring of UAV Components}

1: To begin the wiring phase, the Pixhawk was mounted onto the top of the UAV using industrial strength velcro [32]. A Pixhawk is a user-friendly autopilot system that acts as the "brain" of the UAV. There is an arrow on the Pixhawk (refer to Figure 32) that pointed in the direction determined to be the front of the UAV. 


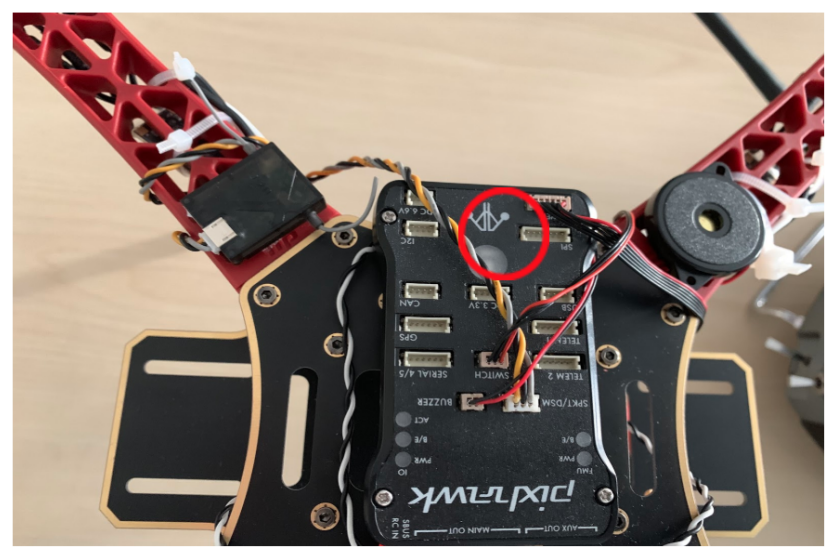

Figure 32: Arrow on the Pixhawk flight controller that points to the front of the UAV.

2: A safety switch was attached to the Pixhawk controller, and secured to the UAV via zip ties. Refer to Figure 33 below. The safety switch ensured that the UAV did not arm until the user was ready and turns off the safety. To turn off the safety, the button at the end of the cable was pressed and held until the light went from flashing red to solid red.

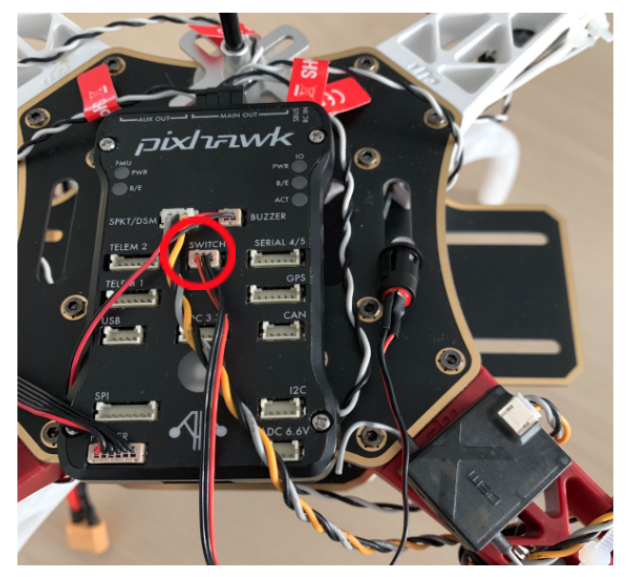

Figure 33: Port on the Pixhawk controller for the safety switch.

3: A buzzer was then attached to the top of the Pixhawk in the slot labeled buzzer, and corresponding apparatus was zip tied down to the closest UAV arm. Refer to Figure 34. This buzzer beeps in meaningful ways that provide information regarding the UAV. For example there is a unique tone for when the UAV arms and disarms. Arming and disarming of the UAV is explained later in this section. 


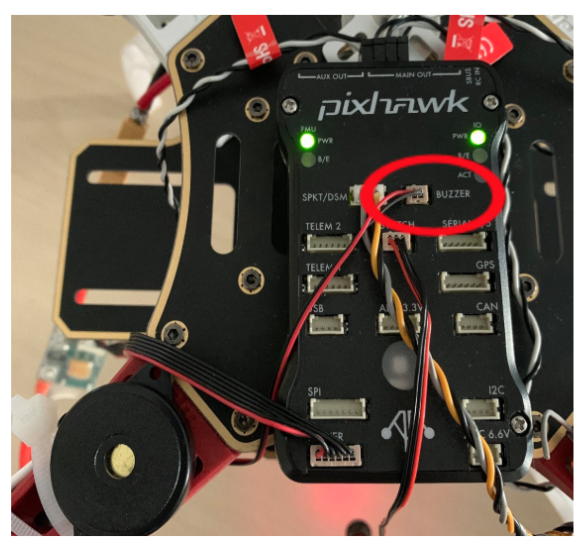

Figure 34: Port on the Pixhawk for the buzzer.

4: The APM Power module was attached to the port labeled "power" on the Pixhawk controller. This module plugs into the battery and provides power to both the motors and the Pixhawk. Refer to Figure 35.

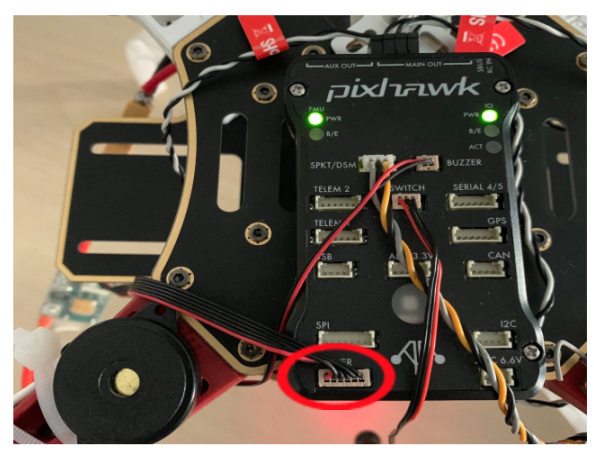

Figure 35: Port on the Pixhawk for the APM power module.

5: Next the cords attached to the motors were plugged into the Pixhawk into the main out slots 1, 2, 3, and 4 as seen in Figure 36. The order in which the plugs are connected to the Pixhawk is depicted in Figure 37. For example, the upper left motor was plugged into slot one on the main out slot on the Pixhawk. 


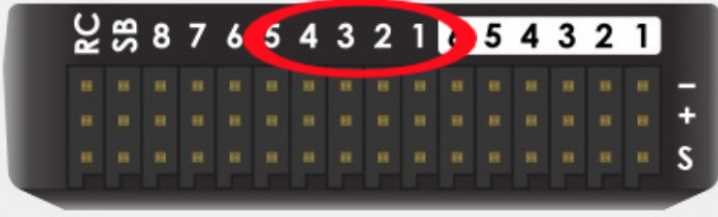

Figure 36: Four main out slots on the Pixhawk controllers into which the motors were plugged [33].
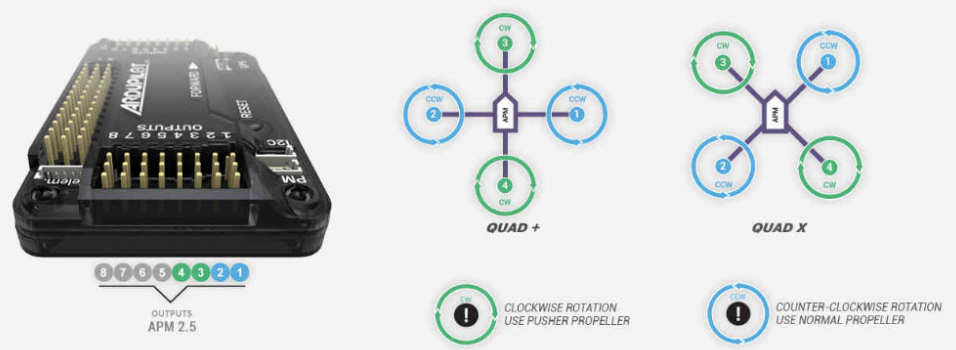

Figure 37: This figure illustrates the numbers of the main out slots on the Pixhawk into which the corresponding motors were plugged [33].

6: The Global Positioning System (GPS) module was then mounted to the UAV. The GPS stand was first secured to the base of the UAV and the GPS module was then secured to the stand using epoxy. Refer to Figure 38 for evidence of the GPS module attached to the stand. 


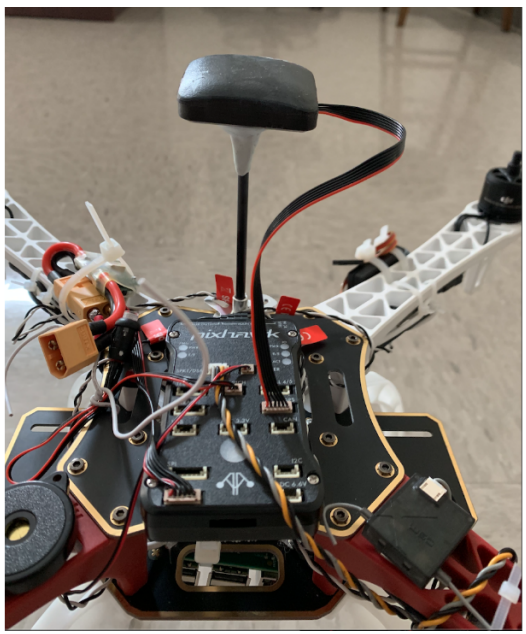

Figure 38: GPS module secured via epoxy to the GPS stand on the UAV.

7: The GPS module was then plugged into the port labeled GPS on the Pixhawk. Refer to Figure 39 for evidence. Note that the arrow pointing forward on the GPS module also aligned with the arrow that pointed to the front of the Pixhawk.

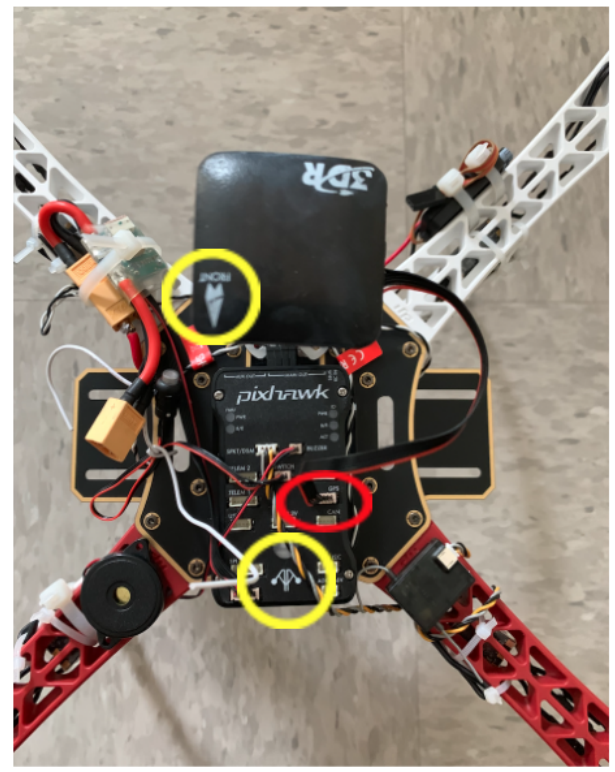

Figure 39: GPS module plugged into the GPS port (circled in red) on the Pixhawk. The arrow on the GPS module pointed in the same direction as the arrow on the Pixhawk (illustrated by the yellow circles). 
8: The receiver was plugged into the port labeled SPKT/DSM on the Pixhawk. Refer to Figure 40.

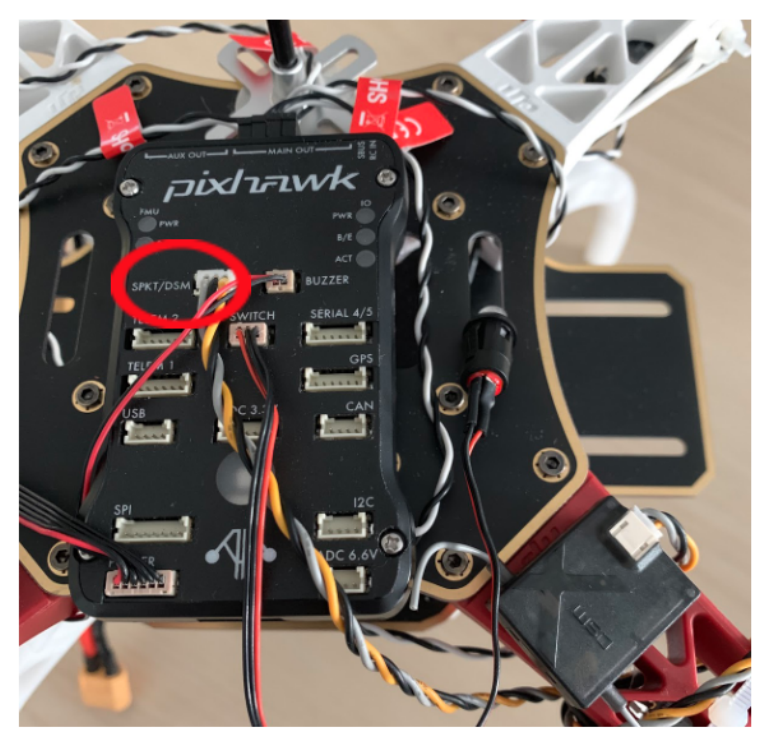

Figure 40: Location on the Pixhawk into which the receiver was plugged.

9: Once the receiver was plugged in, it was bound to the remote control. This process only had to be completed once and then the receiver and remote control remained bound. The first step to binding the receiver and the remote control was to place the bind plug shown in Figure 41 into the last port on the Pixhawk labeled RC in (shown in Figure 42).

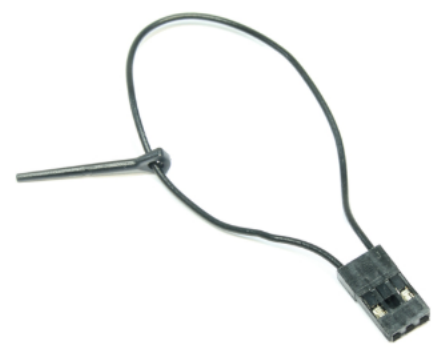

Figure 41: Image of the bind plug that got plugged into the Pixhawk. 


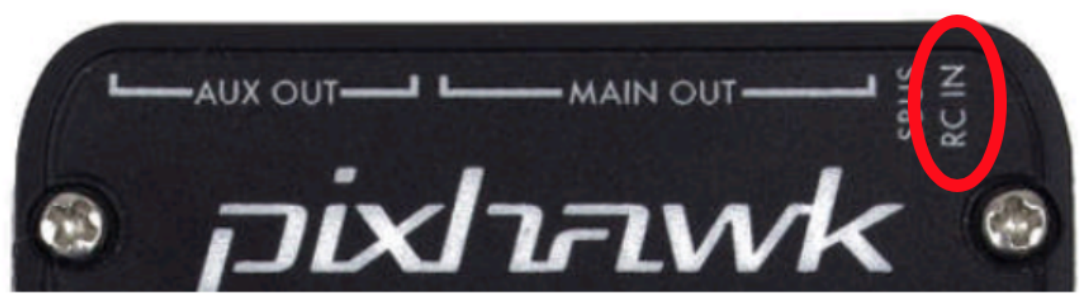

Figure 42: Illustrates the location on the Pixhawk into which the bind plug was inserted.

10: After the bind plug was connected to the Pixhawk, the orange LED light on the DSMX receiver began flashing. This indicated that the receiver was in binding mode.

11: After powering on the Spektrum DX6i remote control, both of the control sticks were held down and to the right position.

12: When the remote control and receiver were done binding, the orange LED on the receiver went from flashing orange to solid orange. The binding process was complete.

\subsubsection{Calibration of Drone}

Note: For these steps, the propellers were taken off the UAV.

1: After the UAV was fully assembled, it was calibrated. The UAV calibration was done via the Mission Planner software. Mission Planner software can be installed onto a Windows PC and was downloaded from the following website: [34].

2: Once the Mission Planner software was installed, it was launched and a Micro-USB to USB cable was used to connect the Pixhawk to the Windows PC. The Micro-USB end of the cord was plugged into the side of the Pixhawk as seen in Figure 43. 


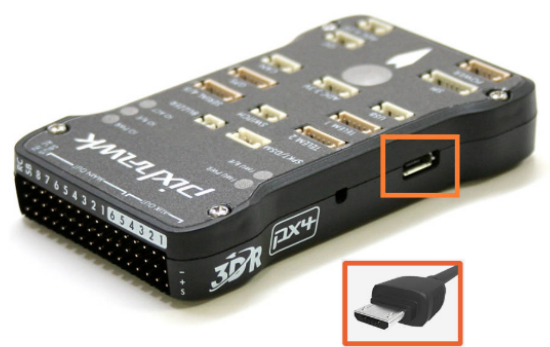

Figure 43: The Micro-USB port on the side of the Pixhawk.

3: In the upper left corner of Mission Planner, there were three drop-down dialogue boxes. In the upper left dialogue box, the "AUTO" option was selected from the drop-down menu. This automatically selected the port enabling the Pixhawk and the computer to communicate. In the upper right dialogue box 9600 was selected. This number represented the baud rate or speed of communication between the Pixhawk and computer. Then the connect button next to the dialogue boxes was selected to connect the Pixhawk to the computer.

4: On the top pane, the "initial setup" option was selected. Under this tab, there are mandatory hardware configurations shown on the side panel as seen in Figure 44. 


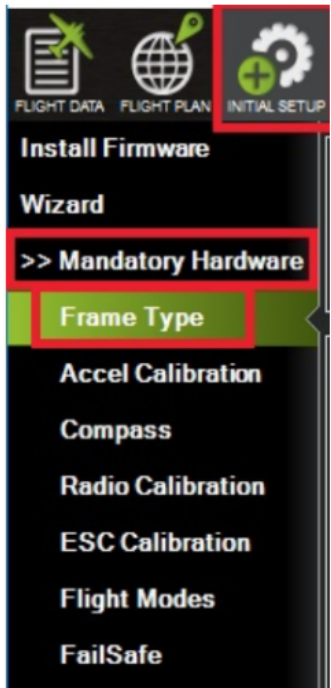

Figure 44: This figure illustrates the mandatory hardware configurations on Mission Planner required to setup and calibrate the UAV.

5: The first step in the setup is the selection of the "Frame Type". The "Frame Type" tab on the side menu was chosen. On the main window, the correct "Frame Class" was selected, which in this case was the quadcopter. Then the "Frame Type" that matched what the UAV looked like was selected from the list of options. Refer to Figure 45 for the proper selections. 


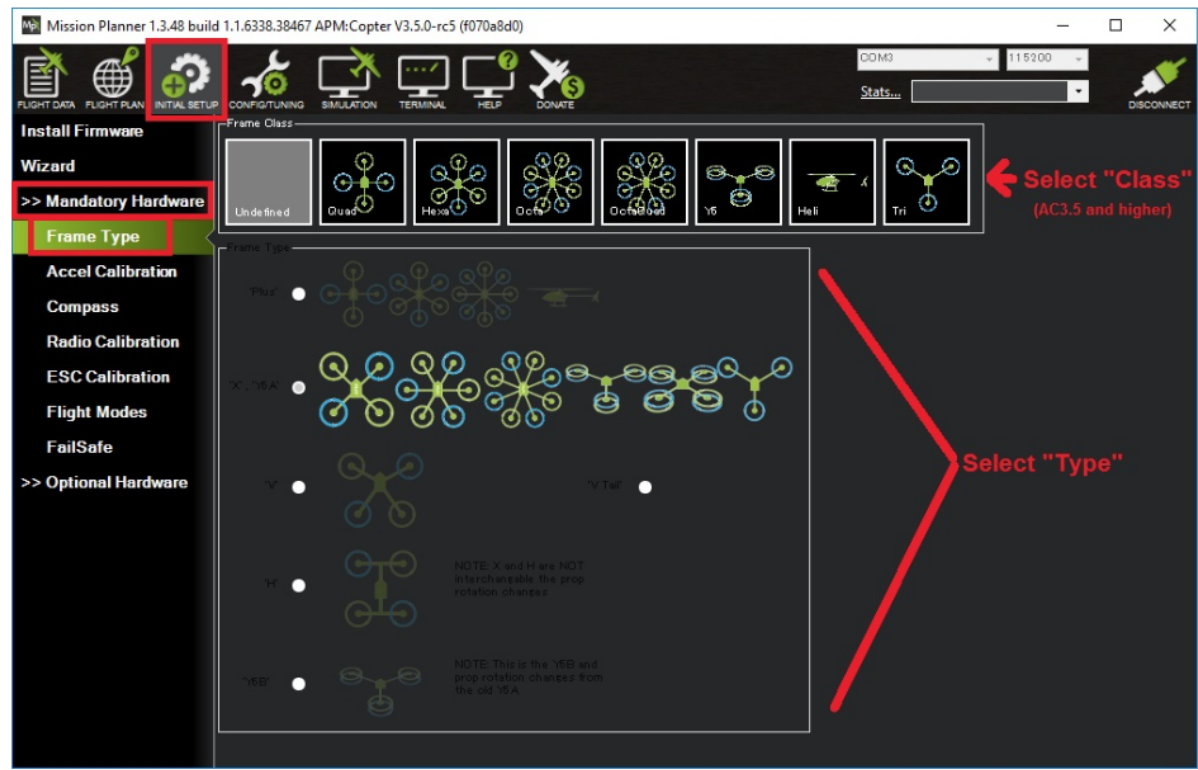

Figure 45: Selecting the frame class and frame type on Mission Planner corresponding to the UAV.

6: Next, the "Accel Calibration" tab was selected to calibrate the accelerometer. The steps to calibrate the accelerometer were followed from the following link: [35].

7: Next, the compass was calibrated. To calibrate the compass, the "Compass" tab on the left hand side of the window was selected. The step-by-step instructions that were followed to calibrate the compass can be found on the following link: [36]. Refer to Figure 46 for evidence that the LED light on the Pixhawk changed to green to indicate that it had a GPS lock. 


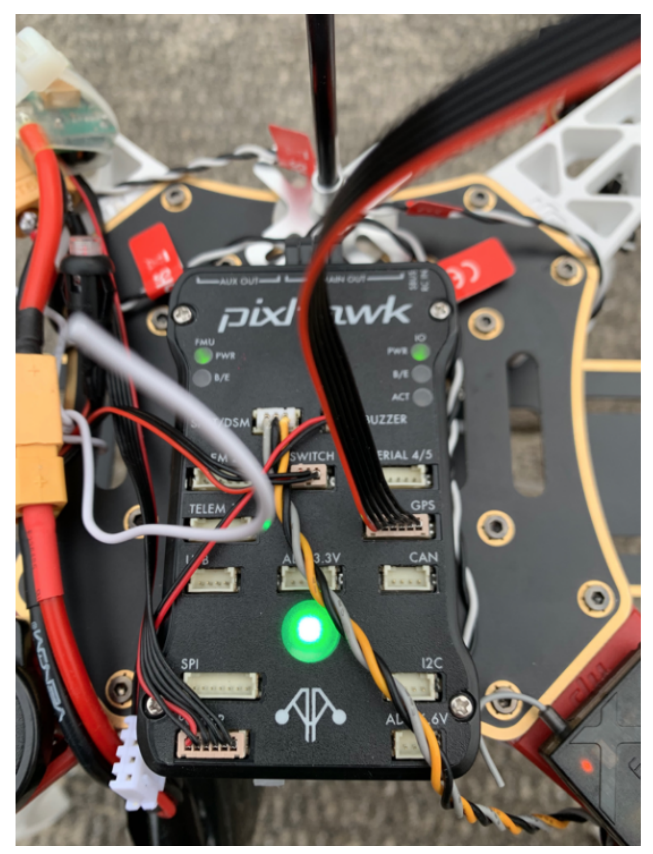

Figure 46: Green LED light on the Pixhawk to indicate it had a GPS lock.

8: Next, the "Radio Calibration" tab was chosen to calibrate the remote control. The step-by-step instructions that were followed to calibrate the remote control can be found on the following link: [37].

9: Then the electronic speed controllers (ECS) that control the spinning of the motors at the speed requested by the autopilot were calibrated. The propellers were not attached during this process. The step-by-step instructions that were followed to complete the ECS calibration can be found at [38].

10: The "Flight Modes" tab was then clicked "on." Nothing was changed on this tab, so the "save modes" button was clicked on the main window to continue.

11: To maintain control of the UAV throughout flight, the FailSafe mechanism was engaged within Mission Planner [39]. To access the FailSafe mechanism within Mission Planner, the "initial setup" option was selected. The FailSafe tab was selected from the left-hand side of the screen (reference Figure 47). 


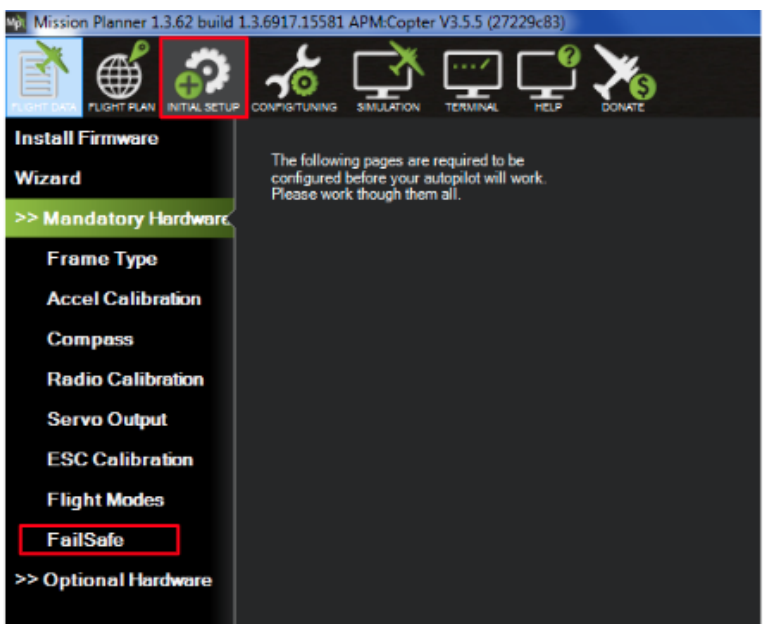

Figure 47: Locating the FailSafe mechanism within Mission Planner.

12: Next, the "Return to Land" (RTL) option was selected for "Battery" and "Radio" (reference Figure 48). If the battery level threatens to fall below $10.5 \mathrm{mAh}$, the UAV will automatically return to the launch location based upon GPS coordinates. Similarly, if the UAV flies beyond the range of communication with the radio controller, the UAV will automatically return to the launch location based upon GPS coordinates. To test the functionality of the FailSafe, consult the following website: [39].

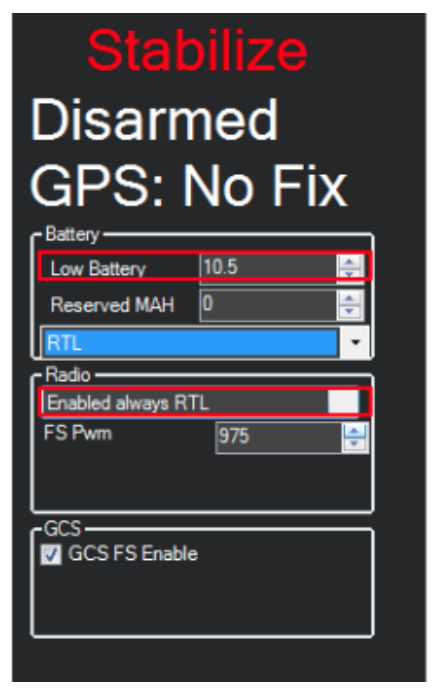

Figure 48: Configuring the FailSafe mechanism within Mission Planner. 


\subsubsection{Starting Up the UAV}

1: Before starting the UAV, the battery had to be sufficiently charged. The battery type used was a Venom $3 \mathrm{~s}$ LiPo battery, meaning that it had three cells. It was charged using a LiPo Battery Balance Charger set to 3.0A and $11.1 \mathrm{~V}(3 \mathrm{~s})$.

a. To charge the battery it was plugged in according to Figure 49 below.

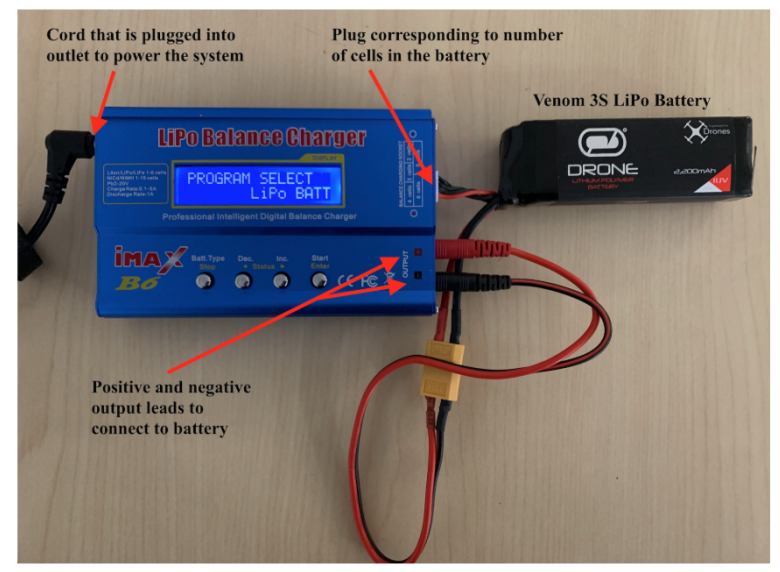

Figure 49: Correct way to plug the battery in to be charged.

b. Program Select -> LiPo BATT, then Start was pressed twice.

c. Once the left value of amperage was blinking, and 3.0A was selected, Start was pressed. The right value of voltage began blinking. The option $11.1 \mathrm{~V}(3 \mathrm{~s})$ was selected.

d. To begin charging the battery, the Start button was held down until the monitor display changed, and then Start was selected again.

e. When the battery was fully charged, a "FULL" message was displayed on the monitor.

2: To start the UAV, the battery was plugged in to power on the UAV. The male XT60 end of the battery was plugged into to the female end of the XT60 plug on the APM power module. The battery was secured between the upper and lower plates using a red Velcro strip provided in the DJI drone assembly kit.

3: As the UAV powered on, the remote control was turned on.

4: The UAV was ready to arm after the LED light on the Pixhawk started 
blinking blue. In order to ensure that the UAV would connect to the receiver, the battery had to be sufficiently charged and the UAV had to be positioned upon a level surface. Refer to Figure 50.

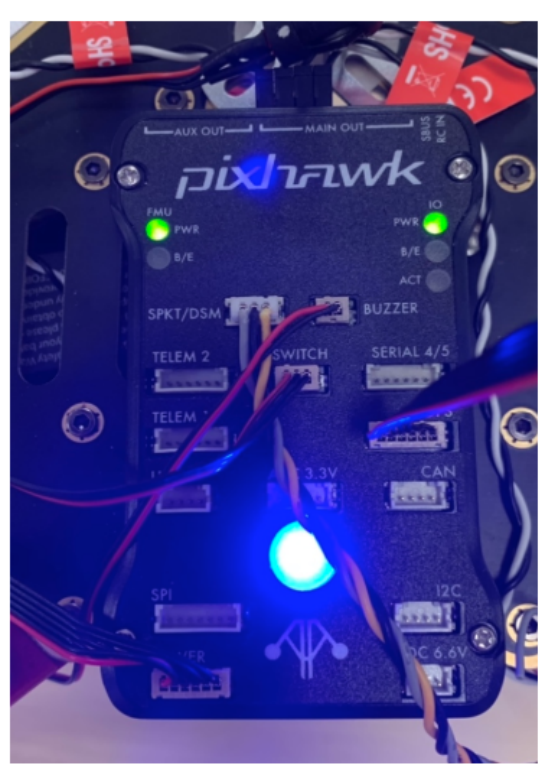

Figure 50: Blue LED on the Pixhawk blinking to indicate the UAV is ready to arm.

5: The UAV was then armed by using the left controller on the remote control and holding it down and to the right as seen in Figure 51. The motors then started turning and the UAV was ready to fly. 


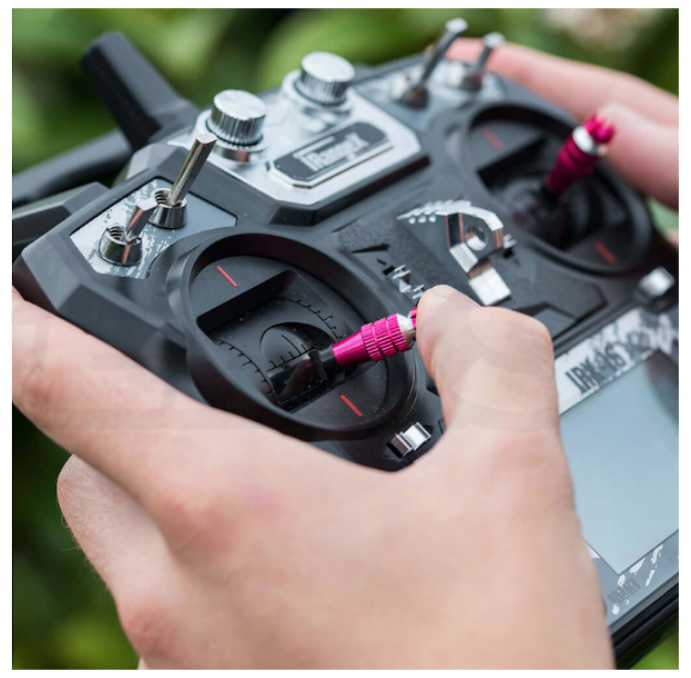

Figure 51: Image illustrating the location the left control stick on the remote control must be in to arm the UAV for flight.

6: The UAV was disarmed by using the left controller on the remote and holding it down and to the left.

\subsection{Results}

The DJI F450, controlled by the Pixhawk autopilot, has the ability to fly. With the addition of a GPS module, the coordinates of the drone's location before taking off as well as the coordinates in flight were recorded in telemetry logs for later viewing. The GPS also enabled a fail safe to be added to the drone so that if it ever flew out of range of the remote control, the drone would return back to the recorded coordinates it logged before leaving the ground.

Additionally, the Raspberry Pi 3 was successfully mounted to the DJI F450 as seen in Figure 31. When trying to find a place to put the Raspberry Pi 3, the experimenters needed to be aware of potential magnetic interference between the Pixhawk Autopilot and the Raspberry Pi 3. As all electronic devices emit a magnetic field, it was imperative that the magnetic fields of the two devices did not interfere and lead to problems with data collection or flying the UAV.

Communication range between the node collecting data and the Raspberry Pi 3 receiving the data was 112.93 meters. This measurement was taken with clear line of site and no interference. While specific testing was not completed to determine how objects impacted range of communication between the Raspberry Pi 0 and Raspberry Pi 3, the authors noted that interfering objects lead to packet loss and decreased range of connectivity when pinging the Raspberry $\mathrm{Pi}$ 
3 device from the Raspberry Pi 0.

\subsection{Discussion}

Assembling a UAV rather than working with an out-of-the box UAV was a valuable experience. A great deal of time was dedicated to troubleshooting problems with the UAV as they occurred, which would not have happened if an out-of-the-box UAV had been used. The time devoted to troubleshooting to create a functional, flying UAV increased familiarity and understanding of the UAV. Working with a UAV that required assembly also forced the experimenters to learn the constituent parts. When parts would break or required parts would be missing, it was imperative to know the exact information about the piece to find replacements and not further delay the project.

Additionally, this project required a unique payload requirement that most preassembled UAVs are not equipped to handle. As previously stated, the experimenters required the ability to mount a Raspberry Pi 3 and portable battery to the UAV to collect data from the ground-based node. As the DJI F450 Flamewheel had room for additional attachments, it was easy to find a home on the UAV for the Raspberry Pi 3. For reference, the bottom of the DJI F450 is shown in Figure 29. The DJI Phantom, a comparable out-of-the-box UAV, is shown in Figure 52. The bottom frame of the DJI F450 had holes into which zip-ties could be fed. The DJI Phantom did not have a thin enough surface that a zip tie could securely wrap around.

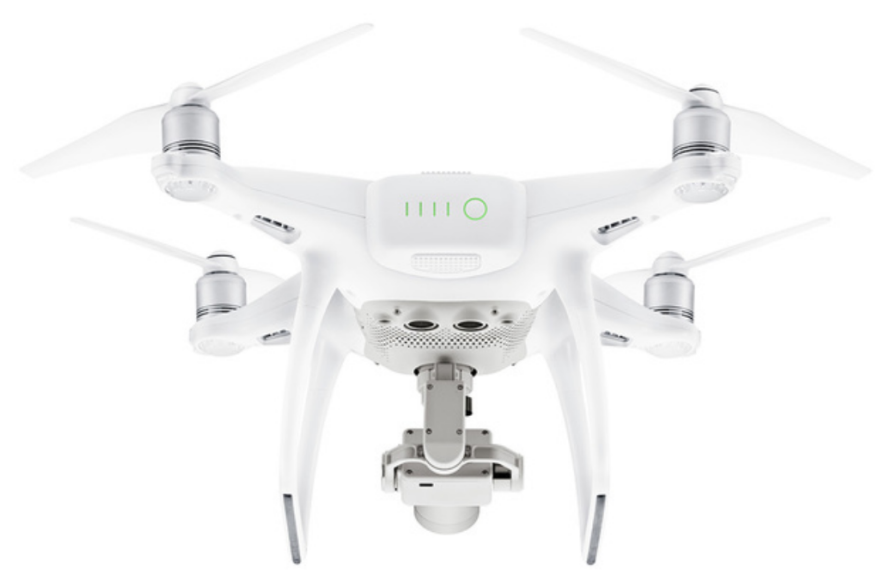

Figure 52: Image of the bottom of a comparable DJI Phantom UAV [33].

While the DJI F450 came with a brain to control the drone, the experimenters found the Pixhawk controller to be more user friendly. When initially working 
with the brain that came with the UAV, there were not many resources on how to properly bind a receiver and a remote control to operate the UAV. As the Pixhawk is more commonly used with UAVs that require assembly, there were many resources regarding how to interface the frame of the DJI F450 with the Pixhawk controller. Additionally, the Mission Planner software that the Pixhawk interfaced with enabled a simple setup and calibration of the UAV. The software walked the user step-by-step through the initial setup phase. Using the Pixhawk and Mission Planner in conjunction also allowed the user to easily troubleshoot issues encountered while attempting to fly the UAV. If there was an error when attempting to arm the UAV for flight, the buzzer attached to the Pixhawk indicated that there was an error with a unique tone. Connecting the Pixhawk to Mission Planner gave a description of the error.

Stakeholders involved with the UAV component of this project include the FAA. The FAA regulates the flying of UAVs. As explained in the section "Public Policy Surrounding Unmanned Aerial Vehicles," restrictions are placed on flying UAVs such as the stipulation that UAVs may not be flown within an hour of civil sunrise or sunset. The FAA has a stake in this problem, as the activities completed for this project must exist abide by regulatory actions. Successful implementation of UAVs for environmental monitoring may be applied to other projects, which will also fall under FAA regulations.

Additionally, organizations such as the South River Science Team, Eastern Brook Trout Joint Venture, and Friends of the Shenandoah River have an interest in the use of UAVs to collect data and monitor watershed conditions. These organizations have a stake in utilizing UAVs to monitor watershed conditions, as time and money are often limiting factors in comprehensive monitoring. Implementation of UAVs to collect data expedites data collection, reducing monetary and time investments. These organizations are largely run by volunteers who dedicate their time to the betterment of the impaired wildlife and habitat of the area. Without their tireless efforts, there would not be a clear understanding of the damage caused by DuPont. It would be significantly more difficult to identify the critical areas in need of restoration. 


\section{Assembling the Wireless Sensor Network}

\subsection{Background Information}

\subsubsection{Wireless Sensor Networks}

A WSN is a network of spatially distributed autonomous sensors which can be used to monitor specific parameters, such as temperature, and transfer the collected data to a main server. A WSN can have military applications, environmental applications, health care applications, and civil applications. A WSN typically consists of two main parts. One part is the sensing system that consists of numerous sensor nodes which communicate amongst each other via radio signal and collect data. The other part is the storage system that is used to perform limited processing on the data and locally store the data gathered from the sensors before forwarding it to a user. Figure 53 displays a basic illustration of a WSN [40].

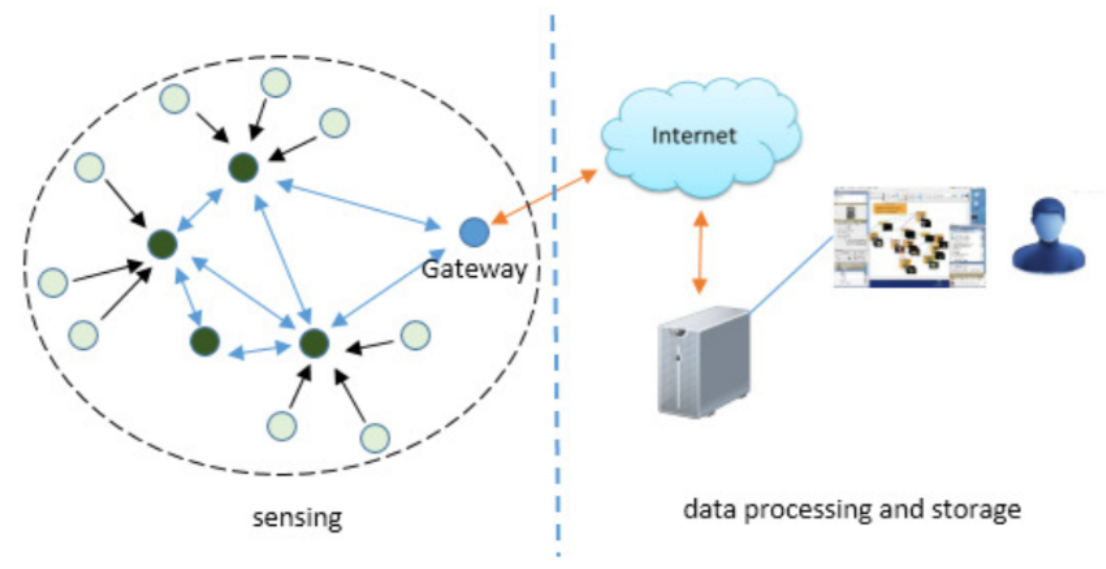

Figure 53: Two basic WSN Components: the sensing system as well as the data processing and storage component [40].

The sensor nodes that make up the sensing system part of a WSN can be split into four groups based on how they function and communicate with the other nodes. The most basic type of sensor node is a sub-node, also known as a leave node or end device. Sub-nodes are nodes without routing capabilities that are only capable of sampling data and transferring this data to other nodes. The second type of sensor is a head-node or a router node. Head-nodes can receive data from other nodes and forward the information. Depending on the configuration of the network, head-nodes can collect data or only be used as a routing point to send data from other nodes. A third type of sensor node is a sink-node or a gateway. The sink-node collects or requests data from other 
nodes and then forwards the data to another network. Finally, the fourth type of sensor node is a controller node or coordinator. The controller node coordinates node addressing, instructs routing, schedules transmission, and synchronizes information between nodes [40]. The node that was used in this project is a sink-node since it collects data from the sensors and forwards it to the Raspberry Pi 3.

WSNs can use various different network topologies but are typically classified as flat or clustered topologies. In flat topologies, all nodes play the same role in the network and therefore all of them are capable of routing the collected data to the server by themselves. In contrast, with clustered topologies, only headnodes can route data and the rest of the nodes will only provide data to the head-node to which they are connected. Refer to Figure 54 for an illustration of a flat topology and Figure 55 for an image of a clustered topology [40].
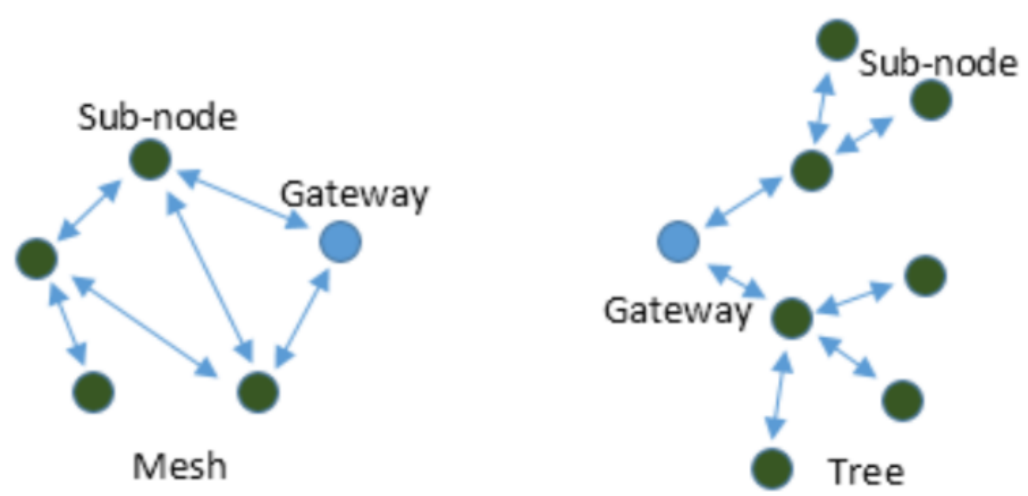

Figure 54: Image depicting a flat topology. As seen in the image, the dark green circles that represent the nodes all play an equal part and are able to send information back and forth amongst each other [40]. 

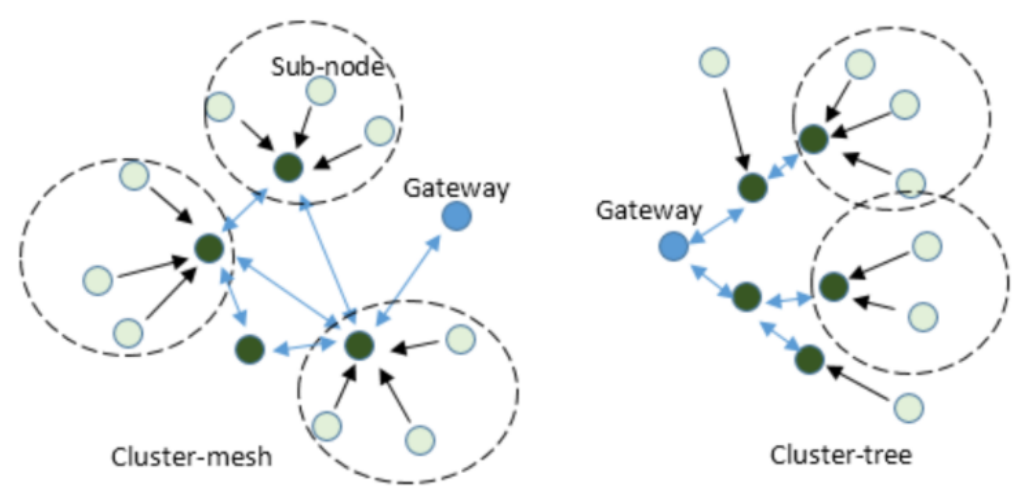

Figure 55: Image illustrating a clustered topology. As seen in the image, the light green circles are only connected to one head-node (in dark green) and send information to that node alone. The head-nodes are capable of communicating with each other and forwarding the information received from the other nodes connected to it [40].

\subsubsection{Systems Model Utilized in this Project}

A WSN will be constructed within Boones Run. Sensor nodes monitoring environmental indicators will be connected to an Arduino microcontroller, and the Arduino will be wired to a Raspberry Pi 0 acting as a sink node. Analog data collected from the sensor will undergo analog-to-digital conversion (ADC) from the Arduino to the Raspberry Pi 0 where it was temporarily stored. The Raspberry Pi 0 will wirelessly transmit the gathered data to a Raspberry Pi 3 or head node acting as an access point on the drone. The Raspberry $\mathrm{Pi} 3$ will store collected data. The end user will then be able to wirelessly send the gathered information to a computer to parse and analyze the data. Refer to Figure 56 for an image of the data flow diagram from the initial sensor node to the end user. 


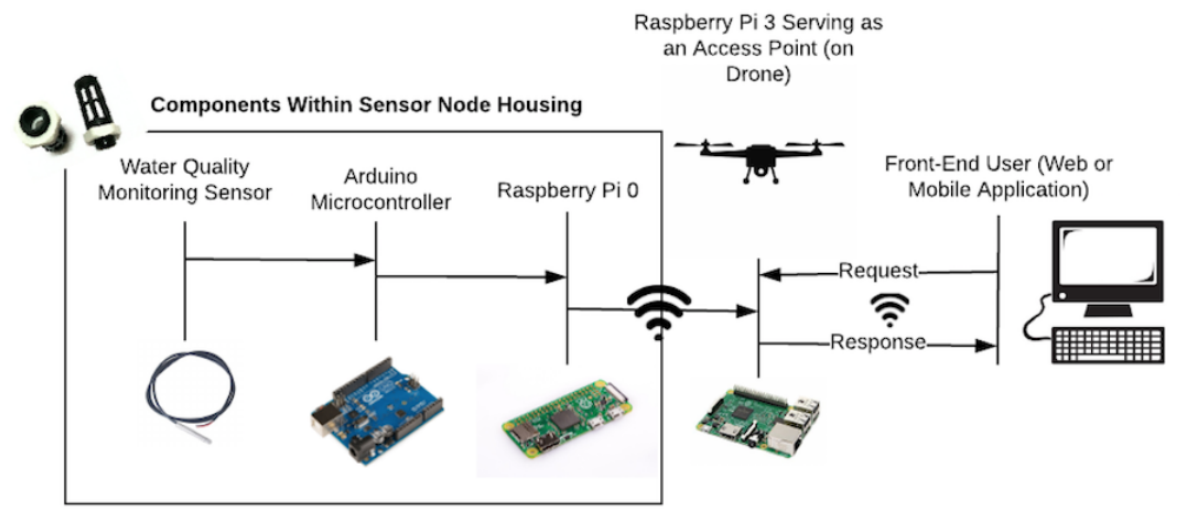

Figure 56: Data flow diagram illustrating flow of information from initial sensor to the end user.

The stated setup of the WSN efficiently and cost-effectively provided a tool to monitor Boones Run water quality.

\subsection{Materials and Methods for Building the Wireless Sen- sor Network}

\subsubsection{Flashing Raspbian onto MicroSD Cards}

The Raspbian image needed to be flashed on two MicroSD cards: one for the Raspberry Ri 0 and one for the Raspberry Pi 3. The steps that were taken to complete this process are described below.

1: The Raspbian Image was downloaded from the website: [41].

2: The MicroSD card adapter was inserted into SD card slot in the iMac computer.

3: Disk Utility was opened on the iMac desktop and the following steps were followed:

a. The name associated with microSD card was selected.

b. Erase was selected. A drop-down menu appeared.

c. The microSD card was renamed.

d. Under format field, the dropdown menu was selected and MacOS Extended Journal was chosen.

e. Erase was clicked.

f. Done was clicked when the erase process was complete. 
4: Next, the command line was opened, and diskutil list was typed. The disk was identified, confirming the disk was available for use by the iMac.

5: The SD card was next unmounted by using the disk identifier to prepare it for copying data: diskutil unmountDisk / dev/disk $<$ disk\# from diskutil $>$.

6: The directory was changed to the location where the Raspbian image was saved.

7: The command sudo dd $\mathbf{b s}=\mathbf{1 m}$ if=image.img of $=/ \mathbf{d e v} / \mathbf{r d i s k}<\operatorname{disk} \#$ from diskutil $>\mathbf{c o n v}=$ sync was entered. Progress of this was checked by clicking Ctrl $+\mathrm{T}$.

8: A success message appeared when it was done loading.

\subsubsection{Enabling Remote Login to Raspberry Pi 3}

1: While the microSD card adapter was plugged into the computer, the directory was changed via cd /Volumes/boot.

2: Next, the command touch ssh was run to create an empty file named "ssh" in the root directory of the SD card. This was done because each time Raspbian boots it enables ssh, if it sees the "ssh" file and deletes the file immediately afterwards.

3: An IP address was automatically assigned to the Raspberry Pi using the following steps:

a. The microSD card was placed into the Raspberry Pi device.

b. A microUSB adapter was used to plug in and power on the device.

c. An ethernet cord was used to connect the Raspberry Pi device via ethernet to the back of the iMac.

d. Internet sharing was then enabled on the iMac by going to System Preferences $>$ Sharing $>$ Internet Sharing and enabling sharing internet from wifi to ethernet. 
4: To find the Raspberry Pi's IP address, connection to the internet was disconnected via removing the ethernet cord and running the command ifconfig | grep "inet" from the command line on the iMac.

5: The ethernet cord was then reconnected to the Raspberry Pi and the following command was run from the iMac.: ifconfig | grep "inet" A new IP address appeared after the Raspberry Pi was reconnected.

6: The first 3 numbers represented the subnet. To find the IP address assigned to the device, a network map was run, 'nmap', on the network the Raspberry Pi was in (ex: nmap -n -sP 192.168.2.255/24).

7: One address was acquired using ifconfig, which was ignored, the other was the address of the Raspberry Pi.

8: The Raspberry Pi could now be remotely accessed. The following command was run on the iMac: ssh pi@ $<\mathbf{i p}$ address of pi $>$. The default password for Raspberry Pi devices is "raspberry".

9: Once SSH'd into the Raspberry Pi, the SSH functionality was permanently enabled by running the command raspi-config.

a. The Interfacing Options menu was selected.

b. SSH was navigated to and selected.

c. Yes was chosen.

d. Ok was selected.

e. Finally, finish was navigated to.

10: SSHing was now permanently enabled on the Raspberry Pi device.

\subsubsection{Changing the Passwords on the Raspberry Pi Devices}

The passwords were changed on the Raspberry Pi devices so that other users could not login to them. Since all of the passwords are initially set to "raspberry", anyone with this knowledge could try to view the scripts and data stored on the Raspberry Pi 3 and 0. Changing the passwords ensured that the information remained secure.

1: The Raspberry Pi device was first remotely logged into using the SSH function on a terminal. "ssh pi@ $<$ ip address $>$ ". If unsure of the Raspberry Pi's IP Address, a nmap of the network was performed using the command 
"nmap 192.168.2.0/24".

2: Once logged in, the command "passwd" was typed to change the password.

3: A prompt to type the current password was displayed (note: the default password for Raspberry Pi devices is "raspberry").

4: A prompt to enter the new password was displayed. The new password was confirmed by typing it again.

5: If successful, the message "passwd: password updated successfully" was displayed.

\subsubsection{Setting up the Raspberry Pi 3 as an Access Point}

1: Ensured that the Raspberry Pi 3 was connected to the internet (WAN).

2: Raspbian was updated with the commands sudo apt-get update and sudo apt-get upgrade

3: The Raspberry Pi 3 was rebooted using the command sudo reboot to ensure the changes saved.

4: DHCPD and HOSAPD software were installed using the command sudo apt-get install dnsmasq hostapd, which enabled the Raspberry Pi 3 to act as the host access point. "y" was typed when prompted.

5: The dnsmasq and hostapd services were stopped using the commands sudo systemctl stop dnsmasq and sudo systemctl stop hostapd.

6: The DHCPD client was configured by opening the configuration file using the command sudo nano /etc/dhcpcd.conf

7: The following lines were added to the end of the dhcpcd.conf file: \# Disable wlan0 so HOSTAPD can start 


\section{Denyinterfaces wlan0}

8: The DHCPCD service was restarted using the command sudo service dhcpcd restart.

9: The dnsmasq configuration file was opened using the command sudo nano /etc/dnsmasq.conf.

10: The following lines were added to the end of the file: interface $=$ wlan dhcp-range $=192.168 .2 .2,192.168 .2 .20,255.255 .255 .0,24 h$

11: The WiFi hotspot with HOSTAPD file was opened and configured using the command sudo nano /etc/hostapd/hostapd.conf

12: The lines seen in Figure 57 were added to the end of the hostapd configuration file. 


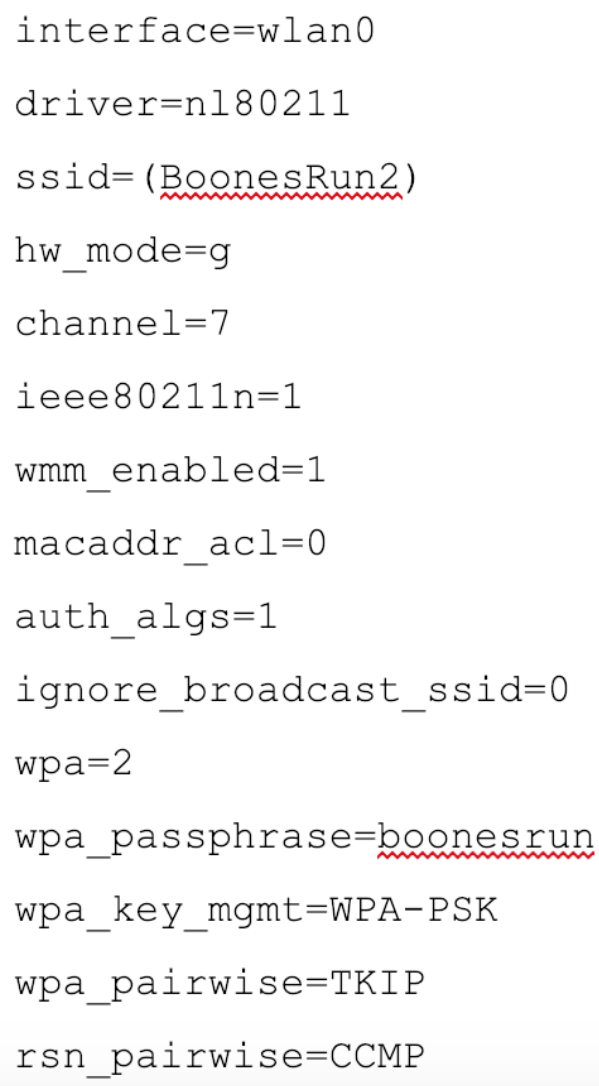

Figure 57: Lines that were added to the end of the hostapd.

13: The access point was manually run using the command sudo /usr/sbin/hostapd /etc/hostapd/hostapd.conf. If the SSID appeared as an available network to connect to, the access point had been successfully established.

14: The hostapd file was reopened to "tell" hostapd where the configuration file was using the command sudo nano /etc/default/hostapd.

15: The DAEMON_CONF file was changed to the following: DAEMON_CONF="/etc/hostapd/hostapd.conf".

16: The hostapd and dnsmasq services were restarted using the commands sudo systemctl start hostapd and sudo systemctl start dnsmasq. 
17: To configure IP routing, first the sysctl configuration file was opened using the command sudo nano/etc/sysctl.conf.

18: The line net.ipv4.ip_forward=1 was uncommented in this file.

19: A masquerade for outbound traffic on eth0 was added with the command sudo iptables -t nat -A POSTROUTING -o etho -j MASQUERADE.

20: The iptable rule was saved using the command sudo sh -c "iptables-save $>$ /etc/iptables.ipv4.nat".

21: To ensure that the above rules were preserved when the Raspberry Pi 3 was booted, the rc.local configuration file was opened using the command sudo nano /etc/rc.local.

22: The lines shown in Figure 58 were added before the exit 0 in the rc.local file.

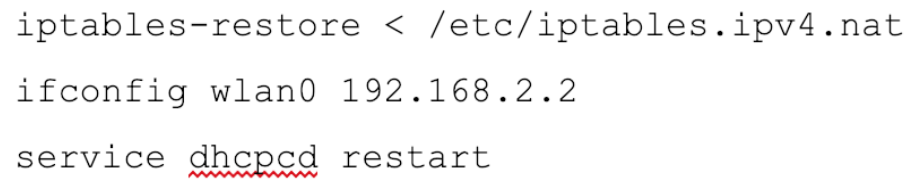

Figure 58: Lines that were added to the end of the rc.local file.

23: The Raspberry Pi 3 was rebooted to ensure the changes worked on the device.

\subsubsection{Downloading and Editing the Arduino Code from the Atlas Website}

The Inter-Integrated Circuit $\left(\mathrm{I}^{2} \mathrm{C}\right)$ Protocol was used to control the communication between the Arduino and the sensors. The $\mathrm{I}^{2} \mathrm{C}$ protocol allowed for ease of communication between up to 127 "slave" devices and a "master" in a single circuit. In this WSN, the slaves were the temperature, conductivity, and $\mathrm{pH}$ sensors, and the master was the Arduino Uno. The $\mathrm{I}^{2} \mathrm{C}$ protocol maintains communication between numerous slaves via a single serial connection because it uses a unique address system and a shared "bus", which is responsible for 
transferring data in a computing system. Each of the sensors had its own address that master "called" before the slave took a reading and transmitted the data. The other communication protocol Atlas had code written for was Universal Asynchronous Reception and Transmission (UART) [42]. The benefit of using the $\mathrm{I}^{2} \mathrm{C}$ over the UART protocol is that UART does not allow for a shared bus system like the $\mathrm{I}^{2} \mathrm{C}$ protocol. Once one of the "slave" devices grabs ahold of the communication bus, it will not release hold of the bus to allow for the other "slave" devices to send data to the "master" [43].

1: The Arduino Linux 64 bits Integrated Design Environment (IDE) environment was downloaded from the Arduino software homepage at the following link: [44].

2: The Arduino $\mathrm{I}^{2} \mathrm{C}$ RTD sample code for the temperature sensor was downloaded from the Atlas Website from the following link: [45].

3: Once the Arduino software was downloaded, the Arduino was connected to a computer via a USB cord, and it was ensured that the power light turned on.

4: The Arduino $\mathrm{I}^{2} \mathrm{C}$ RTD sample code was then uploaded to the Arduino software using the following steps:

a. The Arduino IDE Software was opened. File $>$ Sketchbook $>$ Open was clicked, and navigation was complete to where the downloaded Atlas Temperature Arduino code was saved. That file was uploaded to the environment.

5: Once the file was uploaded and in the environment, the code was presented, along with numerous commented out notes explaining the code and its functionality.

6: Refer to Section 9 Appendix A for the modifications made to the Arduino $\mathrm{I}^{2} \mathrm{C}$ RTD Sample code that enabled continuous data collection the from three sensors (temperature, $\mathrm{pH}$, and conductivity) simultaneously.

\subsubsection{Wiring of the Temperature, $\mathrm{pH}$, and Conductivity Sensors to the Arduino}

1: To wire the temperature, $\mathrm{pH}$, and conductivity sensors to the Arduino, all three were initially wired onto a breadboard to convert them from UART to $\mathrm{I}^{2} \mathrm{C}$ mode. Refer to Figure 59 for the wiring schematic for the temperature 
sensor. Note that while only the temperature sensor is shown in the wiring diagram, all three of the sensors were initially wired in this manner.

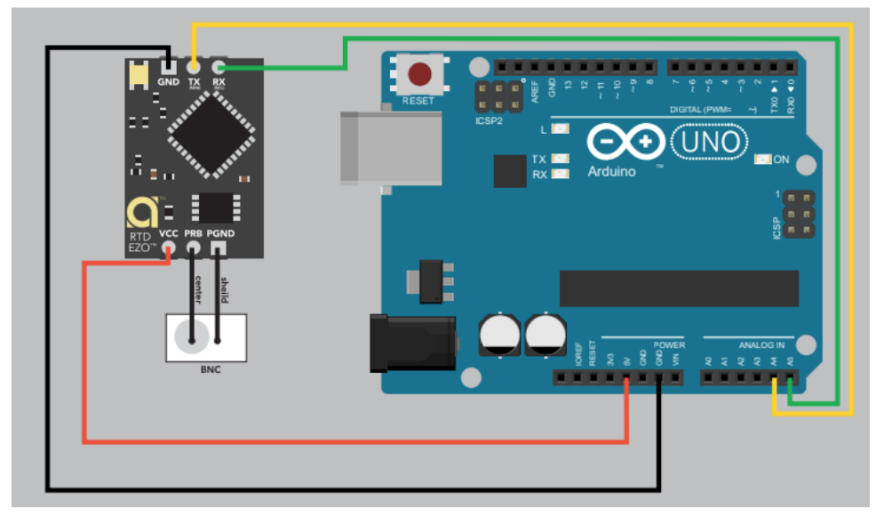

Figure 59: Initial wiring of the temperature, $\mathrm{pH}$, and conductivity circuits to the Arduino Uno to convert to using the $\mathrm{I}^{2} \mathrm{C}$ protocol [45].

2: To switch temperature and $\mathrm{pH}$ circuits to $\mathrm{I}^{2} \mathrm{C}$ mode, the pin connected to the ground was disconnected (powered off).

3: TX and RX were ensured that they were not connected.

4: TX was then connected to PGND as seen in Figure 60.

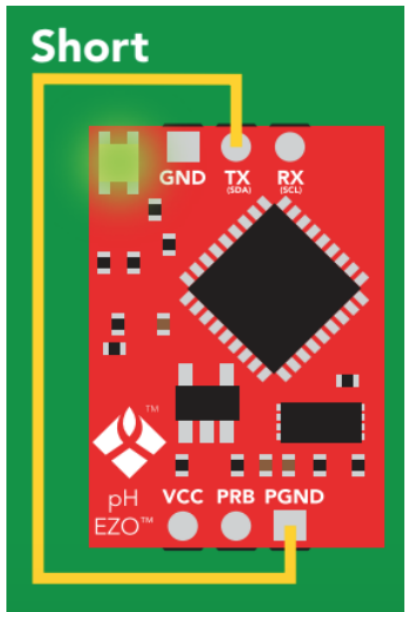

Figure 60: Connection of the TX to the PGND on the temperature and $\mathrm{pH}$ circuits. 
5: The RX was then again confirmed to be disconnected.

6: The GND was then connected to a GND pin on the Arduino.

7: After a few seconds the LED changed from green to blue. This signified that the circuit had been changed to $\mathrm{I}^{2} \mathrm{C}$ mode.

8: The ground was then disconnected again (powered off).

9: Next, the conductivity sensor was manually changed to $\mathrm{I}^{2} \mathrm{C}$ mode. To switch conductivity to $\mathrm{I}^{2} \mathrm{C}$ mode, the pin connected to the ground was disconnected (powered off).

10: TX and RX were disconnected.

11: TX was then connected to the rightmost $\mathrm{PRB}$ as seen in Figure 61.

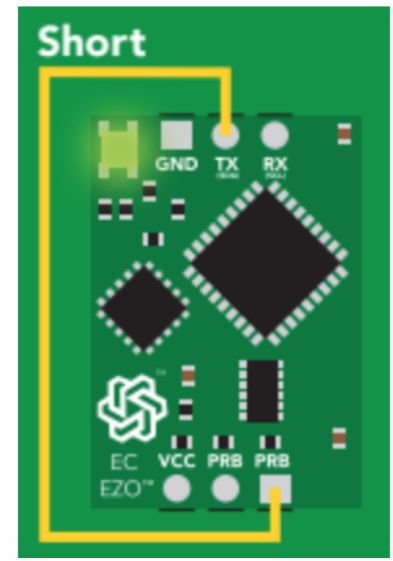

Figure 61: Connection of the TX to the rightmost $\mathrm{PRB}$ on the conductivity circuit.

12: The RX was then again confirmed to be disconnected.

13: The GND was then connected to a GND pin on the Arduino. 
14: After a few seconds the LED changed from green to blue. This signified that the circuit had been changed to $\mathrm{I}^{2} \mathrm{C}$ mode.

15: The sensor circuits were then permanently soldered to a printed circuit board (PCB) and breadboard wiring was utilized to allow for multiple sensor inputs into the A4 and A5 pins on the Arduino, as well all three sensors connected to the $5 \mathrm{~V}$ and ground pins. Using a PCB, the TX and RX inputs from all three sensors were wired into vertical series positions on the breadboard. A connection was made between the series TX on the temperature, $\mathrm{pH}$, and conductivity circuits and pin $\mathrm{A} 4$ on the Arduino. Another connection was made between the series $\mathrm{RX}$ on the temperature, $\mathrm{pH}$, and conductivity circuits and pin A5 on the Arduino. The three sensors were also connected in series on the PCB and connected to a single ground pin on the Arduino Uno. All three sensors were connected again in series from their respective VCC ports. A connection was made from the series VCC on the breadboard to the $5 \mathrm{~V}$ pin on the Arduino Uno. Refer to Figure 62 for a diagram of the wiring on the PCB and the connection of the PCB to the Arduino Uno. Refer to Figure 63 for a close up image of the circuit board with the sensors connected to the Arduino Uno.

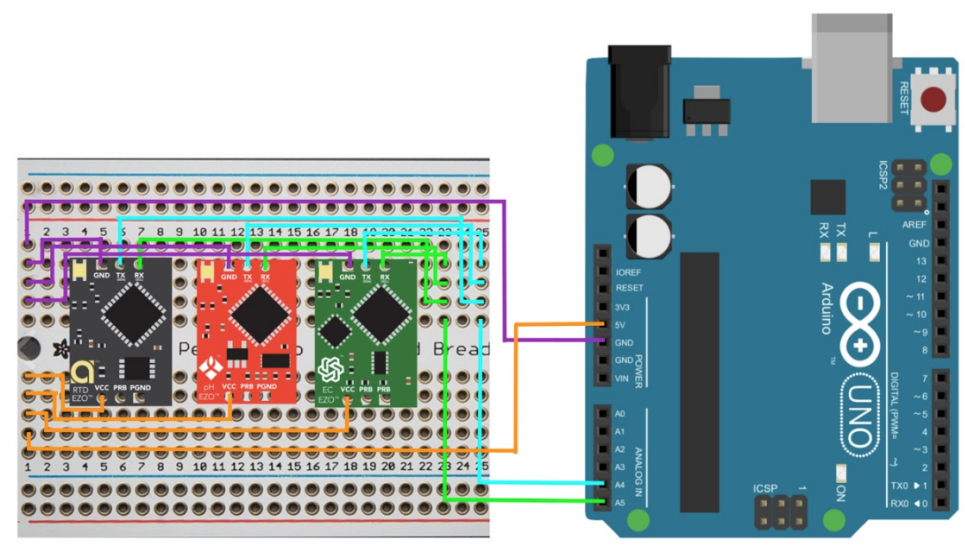

Figure 62: Wiring diagram of the three circuits soldered to the PCB and connected to the Arduino Uno. 


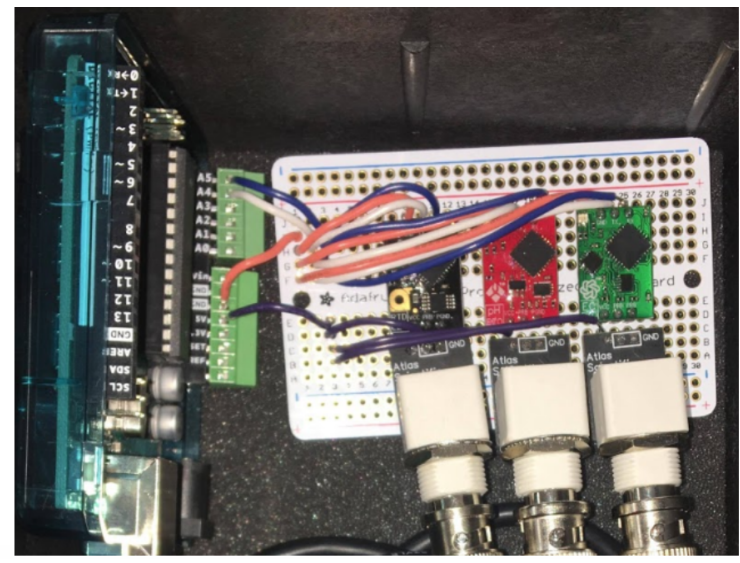

Figure 63: Close-up image of the circuit board with the sensors connected to the Arduino Uno.

\subsubsection{Establishing Communication Between the Arduino and Rasp- berry Pi 0}

1: This code took the data gathered from the sensor wired to the Arduino, parsed it, and added the data to two different .txt files.

2: A terminal was opened and the cd command was used to enter the Desktop directory using the command cd Desktop.

3: To create the Python script to handle the Arduino to Raspberry Pi 0 communication, the command sudo nano arduino_pi01.py was executed. This made a new python file with the name "arduino_pi01".

4: Refer to Section 9 Appendix B to view the code that was entered into the "arduino_pi01" Python file.

5: The data_temp.txt file was a temporary text file that holds the data until it is sent to the Raspberry Pi 3. The data_perm.txt stored all of the data gathered from the sensors as backup.

6: After the Arduino to Raspberry Pi 0 code was written, the Arduino that was wired with the breadboard and sensors was connected to the Raspberry Pi 0 as seen in Figure 64. 


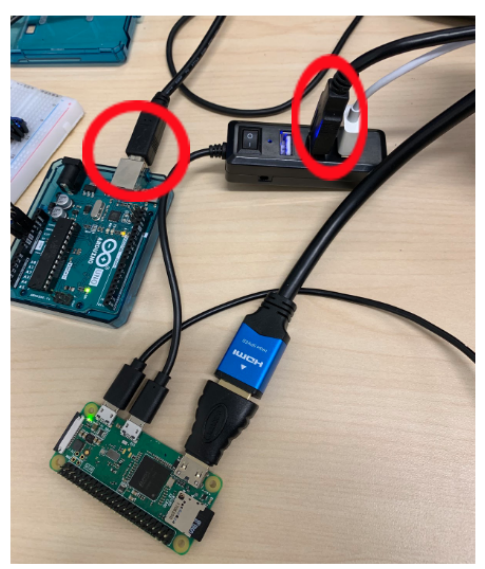

Figure 64: Arduino connected to the Raspberry Pi 0. The USB end of the cord connected to the Arduino was plugged into a splitter that connects to the Raspberry Pi 0.

7: To test that the arduino_pi01.py code was working, the command sudo python arduino_pi01.py was executed on the terminal.

8: After a few data points from the sensors were displayed on the terminal, the arduino_pi01.py code was stopped by pressing "ctrl $+c$ " at the same time.

9: The arduino_pi01.py code then needed to be executed on boot-up of the Raspberry Pi 0 . The command sudo nano /etc/rc.local was executed to edit the rc.local file that runs commands on the boot-up of the Raspberry $\mathrm{Pi}$ device.

10: The command sudo python /home/pi/Desktop/arudino_pi01.py was then added to this file as seen in Figure 65. 


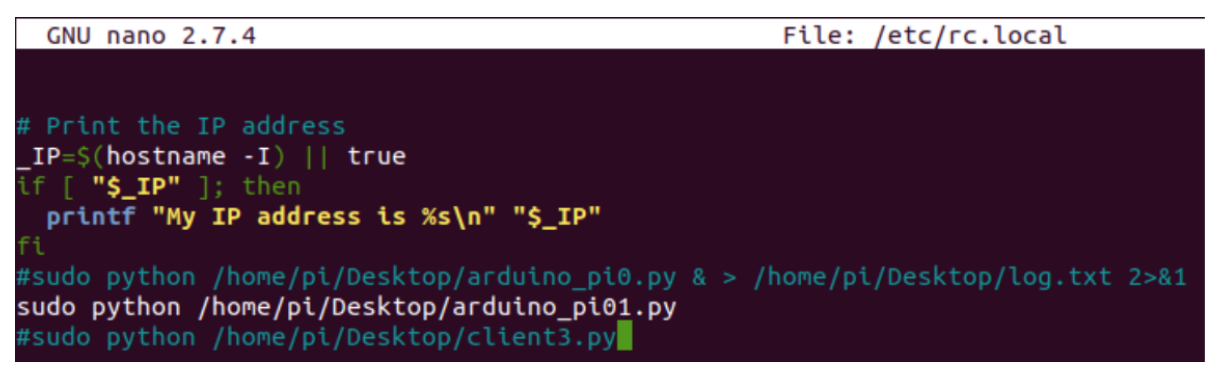

Figure 65: Command that was placed in the /etc/rc.local file to get the arduino_pi01.py code to start on boot up of the Raspberry Pi 0.

11: To allow the changes to save on the Raspberry Pi 0 , the device was rebooted using the command sudo reboot.

12: After the Raspberry Pi 0 device rebooted, it was automatically collecting data points from the sensors connected to the Arduino and storing the data points in the temporary and permanent text files.

13: The devices were left running overnight and the next day the data_temp.txt and data_perm.txt files were checked to confirm the data points were added to these files.

\subsubsection{Establishing Communication Between the Raspberry Pi 0 and Raspberry Pi 3}

Socket coding in Python was used to establish wireless communication between the Raspberry Pi 0 and Raspberry Pi 3. The Raspberry Pi 0 acted as the client and the Raspberry Pi 3 acted as the server. The steps followed to complete this task are shown below.

1: First, on both the Raspberry Pi 0 and the Raspberry Pi 3 it was ensured that Python was downloaded using the command python -version.

2: If Python was not installed, it was installed on both devices using the command sudo apt install python.

3: Next, on both devices, the Desktop directory was entered using the command cd Desktop. 
4: On the Raspberry Pi 0 the command sudo nano client3.py was executed. Refer to Section 9 Appendix $\mathrm{C}$ to view the code that was entered into the client3 file.

5: On the Raspberry 3 the command sudo nano server.py waas executed. Refer to Section 9 Appendix $\mathrm{C}$ to view the code that was entered into the server file.

6: To test, once the client and server code was written, FIRST on the Raspberry Pi 3 the command sudo python server.py was executed to start the server.py file.

7: Once the server.py file was running, on the Raspberry Pi 0 the command sudo python client.py was executed to allow communication between the devices to begin.

8: The client3.py also needed to be executed on boot-up of the Raspberry Pi 0 . The command sudo nano /home/pi/.bashrc was executed to edit the bashrc file file that runs commands on the boot-up of the Raspberry Pi device.

9: The last line of the script was then navigated to and the lines echo Running at boot then sudo python /home/pi/Desktop/client3.py were added to this file.

10: To allow the changes to save on the Raspberry Pi 0 , the device was rebooted using the command sudo reboot.

11: Now that the client3.py file was running at boot, the client3.py file on the Raspberry Pi 0 was able communicate autonomously with the server.py file on the Raspberry Pi 3.

\subsubsection{Physical Construction of WSN Components}

1: After the software components required for the WSN were complete, the ground-based sensor node required physical assembly. The components of the system that were not waterproof (the circuit part of the sensors, the co-axial connector of the sensors, the Arduino, and the Raspberry Pi 0) were placed into a water-resistant Pelican box. A 5/8th inch drill bit was 
used to drill a hole in the side of the box. The probe end of each sensor and the MicroUSB-to-USB cord connecting an iBeek solar battery charger pack to the node were fed through the hole. All of the cords were then fed through a gasket that fit within the diameter of the hole drilled in the box. Refer to Figures 66 and 67 for the setup of the Pelican box with sensors fed through the hole as well as the gasket around the hole, respectively.

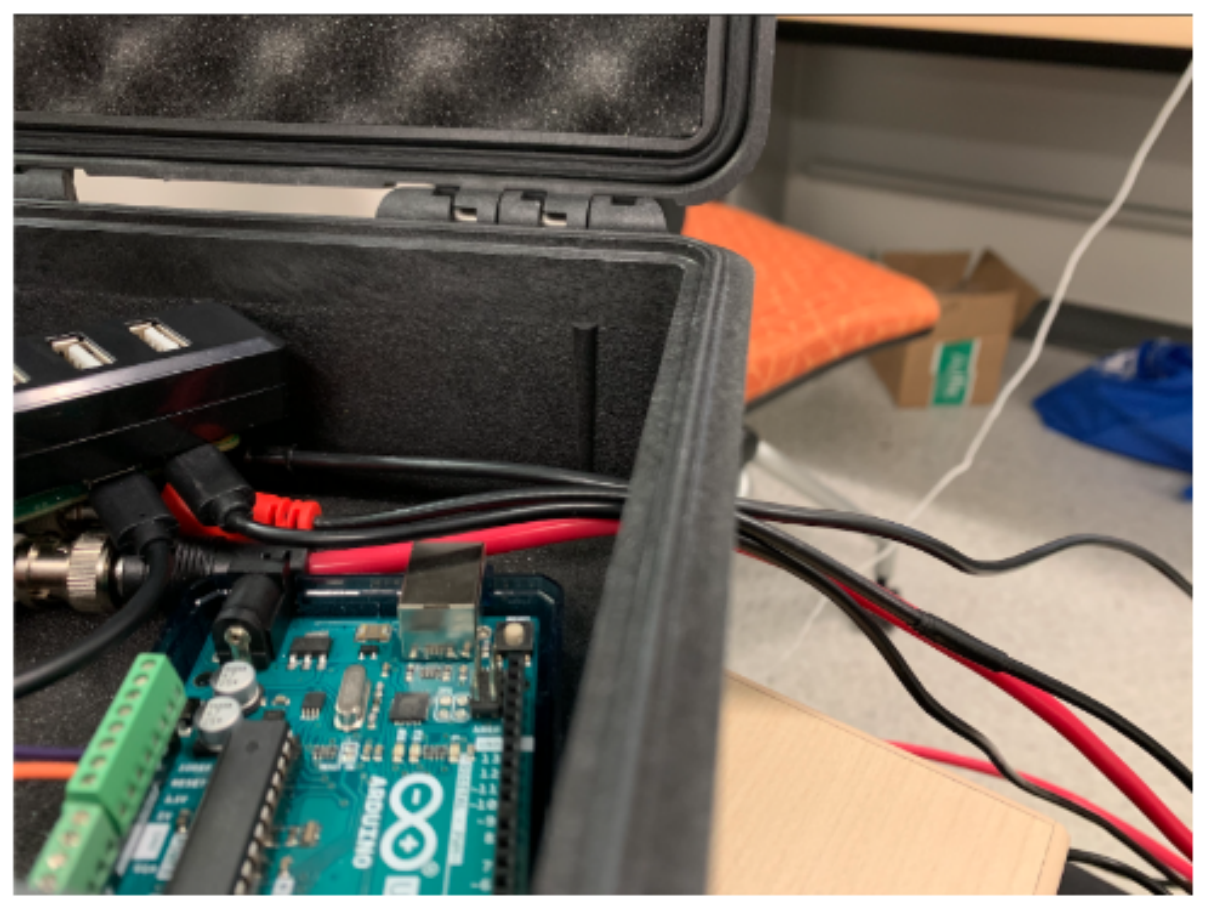

Figure 66: Image of the sensors and MircoUSB-to-USB cord fed through the hole drilled into the Pelican box. 


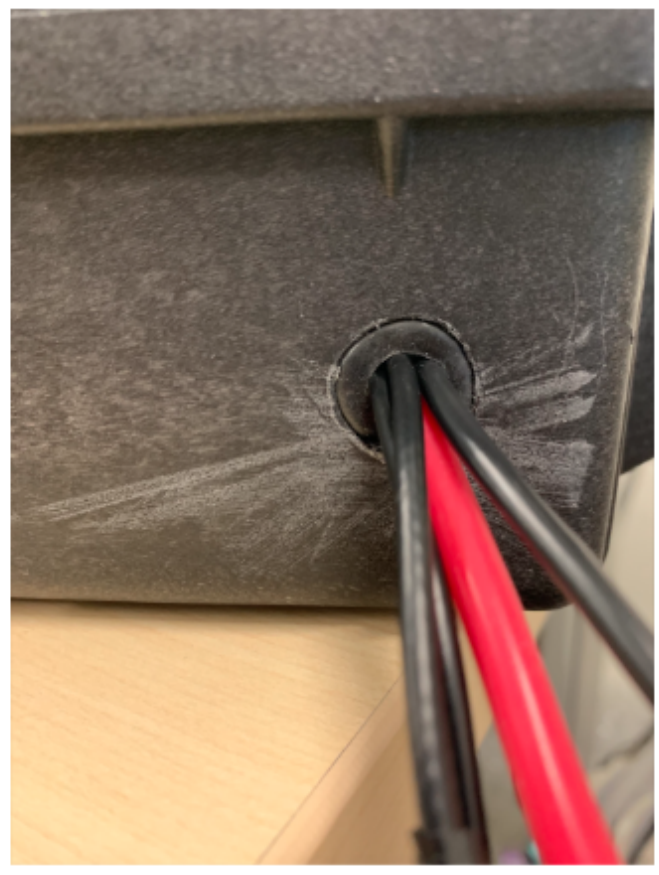

Figure 67: View of the hole drilled into the Pelican box and gauge fitted to the hole.

2: Silicone was used to seal any remaining gaps in the hole drilled into the Pelican Box. The excess cable was taped to the inside wall of the Pelican box to organize the components. The Arduino Uno was placed on its side next to the PCB to which the sensors were attached. Refer to Figure 68 for evidence of the components within the Pelican box. 


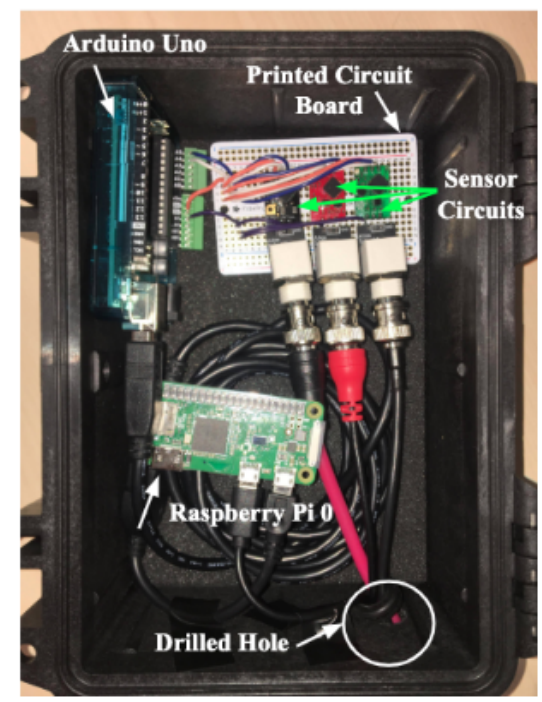

Figure 68: Image of the components inside of the Pelican box.

\subsection{Results}

The successful connection and integration of the multiple parts listed in the materials and methods section above lead to a functioning WSN that was fully automated. The WSN successfully incorporated three different Atlas sensors: temperature, $\mathrm{pH}$, and conductivity. The Arduino that controlled the sampling rate of the three sensors took readings every hour and appended the word "temperature", "pH", and "conductivity" to the end of the reading for ease of distinguishing one reading from the other. The Arduino communicated via serial connection to the Raspberry Pi 0 device. As the Arduino would get a reading, it was sent to the Raspberry Pi 0. A script running on boot-up of the Raspberry $\mathrm{Pi} 0$ parsed the readings it received from the Arduino, added the date and time the data point was gathered, and appended this information to two different text files: a permanent text file that stored all of the data points as backup and a temporary text file that stored the data until it was passed along to the final component of the WSN, the Raspberry Pi 3. The Raspberry Pi 3 successfully operated as an access point that the Raspberry Pi 0 would automatically connect to when it was in range of the Raspberry Pi 3. Ensuring the Raspberry Pi 0 and the Raspberry Pi 3 were in the same network allowed for the two devices to communicate with each other. Using socket coding to enable communication, the Raspberry Pi 0 acted as the client sending the data stored in the temporary text file and the Raspberry Pi 3 acted as the server receiving the data from the Pi 0 . The communication between all parts of WSN allowed the users to mount the Raspberry Pi 3 onto the assembled drone and fly it over the node 
to collect the data in a matter minutes. Refer to Figure 69 for evidence of the data wirelessly transmitted from the node to the Raspberry Pi 3 mounted on the drone.

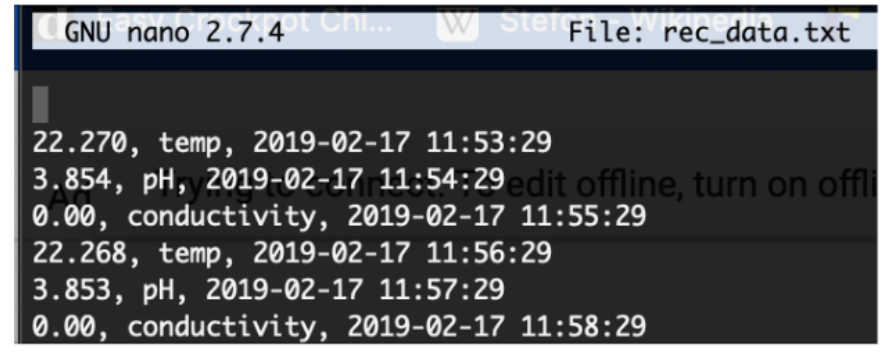

Figure 69: Data wirelessly gathered from the ground-based node displayed on the Raspberry Pi 3.

The total cost of all of the parts required to assemble the WSN and the UAV utilized in this project was approximately $\$ 1,270$. Some of the parts included in this project were only used during assembly or establishing communication, and not included in the node deployed. The cost of replicating the node is approximately $\$ 535$.

The total voltage, current, and power was calculated for the entire node. Refer to Table 8 for the values obtained from these tests.

\begin{tabular}{llll}
\hline Parameter & Minimum Reading & Maximum Reading & Average Reading \\
\hline Voltage (V) & 5.03 & 5.06 & 5.045 \\
Current (A) & 0.23 & 0.33 & 0.280 \\
Power (W) & 1.16 & 1.51 & 1.335 \\
\hline
\end{tabular}

Table 8: Voltage, current, and power drawn from the ground-based node collecting data.

The iBeek solar battery charger pack used to power the node has a 12,000 $\mathrm{mAh}$ capacity battery. Assuming that the battery received no additional charge from the sun, based off the readings received from the node, the battery would be able to power the entire system for 42.86 hours $(12,000 \mathrm{mAh} / 280 \mathrm{~mA})$.

Communication range between the node collecting data and the Raspberry Pi 3 receiving the data was approximately 100 meters. This measurement was taken with clear line of site and no interference. While specific testing was not completed to determine how objects impacted range of communication between the Raspberry Pi 0 and Raspberry Pi 3, the authors noted that interfering 
objects led to packet loss and decreased range of connectivity when pinging the Raspberry Pi 3 device from the Raspberry Pi 0.

\subsection{Discussion}

The implementation of the UAV enabled WSN used in this project served as a successful proof of concept to validate its applications and usefulness in remotely gathering environmental data.

As explained in the previous results section, the entire node drew an average of 5.045 volts, 0.280 amps, and 1.335 watts. The node created compared most similarly to a small hand-held calculator. A TI-84 Plus C Silver Edition calculator drew 5.03 volts, 0.18 amps, and 0.90 watts. The node drew more current than the calculator by a factor of 1.5. The node drew approximately the same amount of voltage as an iPhone XS as the iPhone drew 5.04 volts. However, the iPhone drew more current and power than the node at values of 0.99 amps and 4.98 watts. The node also drew a similar value of voltage compared to an iPad third generation tablet device as the tablet also drew 5.04 volts. Like the iPhone, the tablet also drew more current and power than the node at values of $0.97 \mathrm{amps}$ and 4.88 watts. The current drawn by the node was significantly less than that of the iPhone and the iPad by a factor of a little over 3.5. Refer to Figure 70 for a graphical comparison of these values.

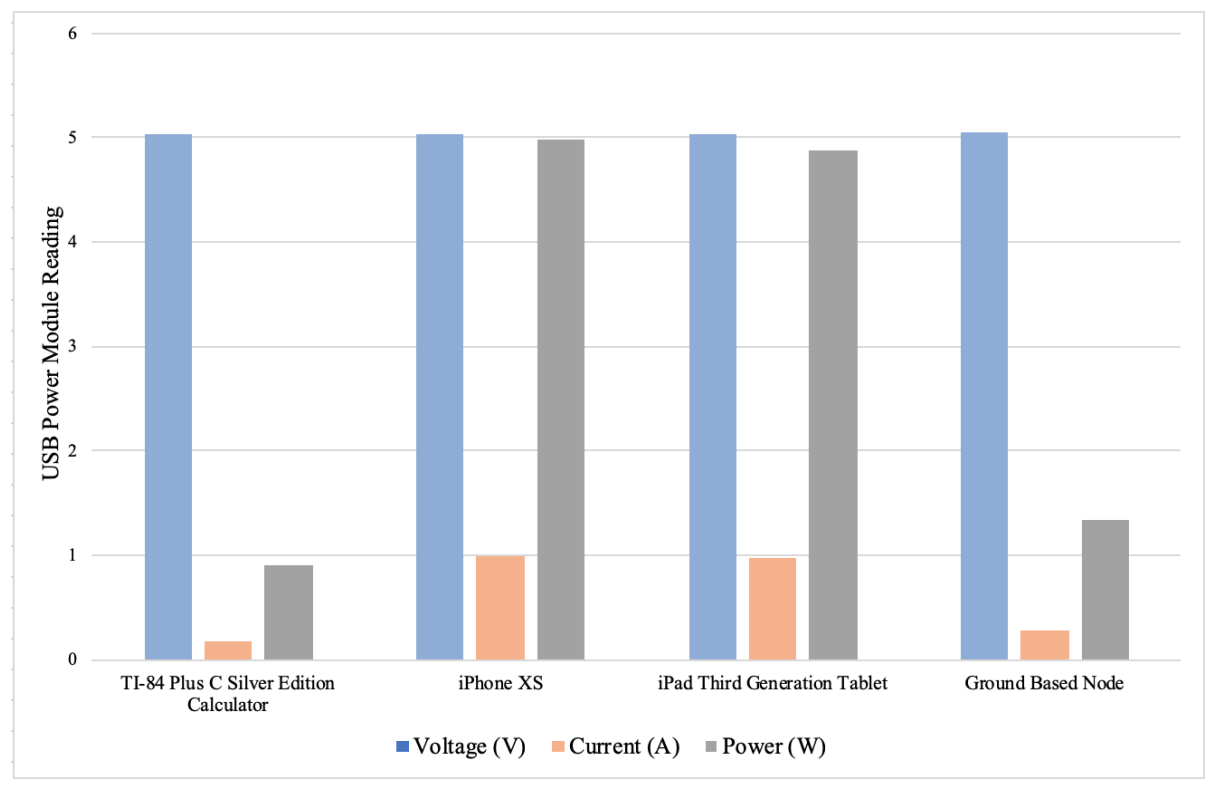

Figure 70: Comparison of power consumption of small handheld devices in comparison to the ground based sensor node created for this project. 
If the iPhone and iPad tablet were to be continuously powered by the same solar battery pack that was used to power the node, each would only be powered for 12.12 hours and 12.37 hours, respectively. As the number of amps the node drew was relatively low, the power demand placed on the battery was not as significant as other handheld devices.

One major benefit of utilizing the UAV enabled WSN was the reduction of data collection time in comparison to traditional hand sampling methods. On average, approximately three to four hours were spent collecting and reading the data using traditional methods. Flying the UAV over the location where the node was to gather data was estimated to take between 10 and 15 minutes. However, it is important to note that this value is an incomplete view of the total time required. This value only considered the time it took to fly the UAV over the node and wirelessly collect the data. When the node is actually implemented, time taken to drive out to the site would also need to be included in this estimation. The time taken to collect data by hand is included in the three to four hours estimation previously mentioned. Therefore, the current comparison is not one to one. Additionally, only one individual was required to fly the drone over the node to gather the data. When sampling the data by hand, two to three people were required to take the actual readings, record the values, and take any site notes.

Finally, the experimenters speculated that system established in this project would pay itself off after one year. As previously noted, the upfront cost of establishing the entire system is approximately $\$ 1,270$. After one WSN has been established with a UAV, the cost of replicating another node would be approximately $\$ 535$. The average median salary of environmental scientists is $\$ 55,730$ or an hourly rate of $\$ 26.80$ [46]. With an average traditional data collection time of 3.5 hours, once a month for a year, the money required to pay this individual would be approximately $\$ 1,125$ for the year. The difference between the initial upfront cost of building the entire system versus using hand sampling methods is $\$ 145$. The following year - once the system is in place - would be a zero cost, excluding any required maintenance to the system. Assuming that there is in fact a decrease in the amount of time taken to gather data from the node, the cost of system described in this project would break even with the cost of hand sampling methods the next year. 


\section{Future Work}

One step that could be taken in the future is adding another testing location once the Boones Run property changes hands and becomes public land. As explained earlier, the experimenters were only able to test in parts of the stream that were accessible via public roads so as not to trespass on landowners properties. However, once the land changes hands and becomes public, future experimenters will have access to parts of the stream that were inaccessible throughout the extent of this project. Adding this fourth testing location will allow future experimenters to have a more holistic view of the condition of Boones Run and better monitor its progress throughout the forthcoming restoration process. Furthermore, additional parameters could be monitored such as water level and turbidity to assist in gauging the health of the stream.

The Pixhawk autopilot that was used when assembling the drone has the ability to follow flight plans. Once a flight path is well known and has been confirmed to pose no risk to the drone in these heavily vegetated testing areas, this functionality can be utilized for additional ease of data collection. The drone could also be used to take aerial imagery of the different sites. Testing would have to be conducted to determine how adding the additional payload of the camera would impact the drone's battery, but this imagery could be used for further analysis of the testing locations.

Another step that could be taken in the future to advance this project would be to add security protocols during data transfer between the Raspberry Pi 0 and Raspberry Pi 3. While the data gathered during this project was not extremely important to protect, should this setup be applied to collect data in a more sensitive environment, proactive measures should be taken to reduce potential security breaches and loss of data. Currently, the data is stored and sent as plain text, so one way to make the data more secure could be to hash the stored data. Additionally, public key cryptography could be used to secure the collected data. This would allow for data to be encrypted with the intended receiver's public key (or a key that can be obtained and used by anyone for encryption) but the encrypted message can only be decrypted by that receiver's private key (or key that is only known to the recipient).

While only one node with three sensors was utilized in this project, future work could involve researching and testing considerations for more than one node in close proximity. Adding another node in close proximity to the first node would come with increased complications such as both nodes trying to communicate with the Raspberry Pi 3 when it is within communication rage. Testing would have to be done to determine how far apart the nodes would have to be so that both of them do not attempt to send data to the Raspberry Pi 3 at the same time. Additionally, more information would have to be added in the text files to uniquely identify the locations of the nodes so the data does not get mixed up. 
A final step that could be taken to advance the project would be to update the Arduino Uno code that controls the three sensors. The way the code is written as of right now, a reading is taken from one sensor, it waits an hour then takes a reading from the second sensor, then waits another hour to take the reading from the third sensor. This process is then repeated infinitely. As it is more comprehensive to have the three readings taken simultaneously, the Arduino Uno code should be edited for this to be accomplished. The edit would take all three readings from the sensors simultaneously, wait an hour then take all three readings again. 
Potter, Valentino, \& Yates

\section{Acknowledgments}

Help was received from numerous individuals throughout the course of this project. The experimenters would first and foremost like to acknowledge their advisors Dr. Thomas Benzing and Dr. Ahmad Salman for their guidance throughout this project. The success of the project was due to their dedication and willingness to help the experimenters when needed. The experimenters would also like to acknowledge Dr. Samy El-Tawab for his assistance and expertise in the $\mathrm{C}$ coding language. Safaa Al Badry provided the experimenters with much of computing the hardware required for the project including the computers, Arduinos, Raspberry Pi devices, and cords. The experimenters would also like to acknowledge Kyle Snow for assisting with the operation of the WTW Meter used to gather data by hand. Kyle also ensured all the the equipment that was needed during sampling was set up and ready for the experimenters before going out in the field. The experimenters would also like to acknowledge Fred Briggs for his expertise in drone assembly and operation. Fred dedicated time out of his busy schedule to sit down with the experimenters and determine why the drone was initially not flying. Nick Swayne and the JMU X-Labs also let the experimenters borrow missing parts required for the drone. The experimenters would also like to acknowledge the ISAT funds appropriation committee for allocating money to allow the experimenters to purchase additional equipment that they did not already have on hand. Whitney Sites assisted the experimenters in ordering the additional parts needed for the project. Finally, the experimenters would like to acknowledge the JMU Rose Library Makery for training the experimenters on how to solder parts as well as assist in troubleshooting Arduino code. 


\section{Lab References}

[1] U. S. EPA. (2004). 2004 national water quality inventory report to congress, [Online]. Available: https://www.epa.gov/waterdata/2004-nationalwater-quality-inventory-report-congress (visited on 04/01/2019).

[2] D. Myers. (2016). Why monitor water quality? [Online]. Available: https: / / water . usgs . gov / owq / WhyMonitorWaterQuality · pdf (visited on 04/26/2018).

[3] South River Science Team. (2018). Historical timeline, [Online]. Available: http://southriverscienceteam.org/timeline/.

[4] U. S. EPA. (2017). Mercury, elemental, [Online]. Available: https : // cfpub.epa.gov/ncea/iris2/chemicalLanding.cfm?substance_nmbr= 370.

[5] South River Science Team. (2009). People, mercury, and the river, [Online]. Available: http : / / southriverscienceteam . org / news / fact sheets/SR_No2.pdf.

[6] Virginia Department of Health. (2018). Fish consumption advisory, [Online]. Available: http://www.vdh.virginia.gov/epidemiology/epidemiologyfact-sheets/mercury/ (visited on 04/01/2019).

[7] M. Strickler. (2018). Dupont nrdar settlement, [Online]. Available: https : / / naturalresources . virginia . gov / dupont-settlement-grants/ (visited on 04/13/2018).

[8] (). Boone run at rt 636 near elkton, va (usgs-0162909150) site data in the water quality portal, [Online]. Available: https://www . waterqualitydata. us/provider/NWIS/USGS-VA/USGS-0162909150/.

[9] U.S. Fish and Wildlife Service. (2014). Endangered and threatened species in virginia, [Online]. Available: https : / /www . waterqualitydata.us / provider/NWIS/USGS-VA/USGS-0162909150/.

[10] Virginia Department of Game and Inland Fisheries. (). Vafwis search report, [Online]. Available: http://vafwis . org $/ \mathrm{f}$ wis $/$ ?Title=VaFWIS $\% 5 \mathrm{C} \%$ 2BGeographicSelect $\% 5 \mathrm{C} \% 2 \mathrm{BOptions} \&$ poi $=38 \% 5 \mathrm{C} \% 2 \mathrm{C} 26 \% 5 \mathrm{C} \% 2 \mathrm{C} 23.4 \% 20-$ $78 \% 5 \mathrm{C} \% 2 \mathrm{C} 38 \% 5 \mathrm{C} \% 2 \mathrm{C} 03.0 \&$ dist $=4828.032 \&$ report $=\mathrm{V} \& \mathrm{placeName}=$ Boone $\%$ 20Run $\% 20 \% 5 \mathrm{C} \% 28$ Stream $\% 5 \mathrm{C} \% 29 \% 5 \mathrm{C} \% 3 \mathrm{~B} \% 20$ Rockingham\&lastMenu=Home . _ - By $\% 5 \mathrm{C} \% 2 \mathrm{BPl}$ ace $\% 5 \mathrm{C} \% 2 \mathrm{BName} \& \mathrm{dt}=$ February $\% 5 \mathrm{C} \% 2 \mathrm{~B} 08 \% 5 \mathrm{C} \% 2 \mathrm{C} \% 5 \mathrm{C} \%$ 2B2018\%5C\%2B2\%5C \%3A11\%5C \%3A32AM (visited on 02/05/2018).

[11] L. Peters. (Sep. 28, 2016). The legacy of the south river, [Online]. Available: https://www . newsleader. com/story/news/local/2016/09/28/ legacy-south-river/91218268/ (visited on ).

[12] Waynesboro Public Works. (). Wastewater treatment plant, [Online]. Available: https : / /www . waynesboro . va . us /305/Wastewater-TreatmentPlant (visited on ). 
[13] (2010). 2vital signs: The five basic water quality parameters, [Online]. Available: http://home.iitk.ac.in/ anubha/Water2.pdf (visited on 04/27/2018).

[14] NIWA. (). Habitat indicators of stream health, [Online]. Available: https: //www.niwa.co.nz/our-science/freshwater/tools/shmak/manual/ 9habitat (visited on 04/27/2018).

[15] - (). Virginia legislative code, [Online]. Available: https://www .niwa. co . nz / our-science / freshwater / tools / shmak / manual / 9habitat (visited on $04 / 27 / 2018$ ).

[16] U.S. EPA. (2012). Water: Monitoring \& assessment., [Online]. Available: https : //archive .epa.gov/water/archive/web/html/vms511 .html (visited on $04 / 27 / 2018$ ).

[17] IDEXX. (). Overview, [Online]. Available: https://www.idexx.com/en/ water/water-products-services/colilert/ (visited on 04/01/2019).

[18] —, (2019). Idexx quanti-tray $® / 2000$ mpn table ( per 100ml), [Online]. Available: https://www.idexx.com/files/qt97mpntable.pdf (visited on $04 / 03 / 2019)$.

[19] M. Online. (). Imost probable number (mpn) test: Principle, procedure and results, [Online]. Available: https ://microbeonline .com/probablenumber-mpn-test-principle-procedure-results/ (visited on 04/03/2019).

[20] United States Environmental Protection Agency. (2016). Surface waters information, [Online]. Available: https : / / archive . epa . gov/ emap / archive-emap/web/html/index-43.html (visited on 04/01/2019).

[21] United States Environmental Protection Agency. (2017). History of the clean water act, [Online]. Available: https : / / www . epa . gov / laws regulations/history-clean-water-act (visited on 04/01/2019).

[22] Virginia Department of Environmental Quality. (2018). Permitting and compliance, [Online]. Available: https : / / www . deq.virginia . gov / Programs/Water/PermittingCompliance. aspx.

[23] Virginia Administrative Code. (2017). Numerical criteria for dissolved oxygen, ph, and maximum temperature, [Online]. Available: http : / / lis . virginia . gov/cgi-bin/legp604 . exe?000+reg+9VAC25-260-50 (visited on $04 / 01 / 2019$ ).

[24] W. R. Center. (). E coli in water, [Online]. Available: https : / /www . water-research . net/index . php / e-coli-in-water (visited on 04/03/2019).

[25] P. M. Jakus and W. D. Shaw, "Perceived Hazard and Product Choice: An Application to Recreational Site Choice.," Journal Of Risk $\mathcal{E}$ Uncertainty, vol. 26, no. 1, pp. 77-92, 2003. DOI: https ://doi.org/10.1023/A : 1022202424036 . 
[26] C. Trasvina-Moreno, Blasco, Ruben, A. Marco, Casas, Roberto, and A. Trasvina-Castro, "Unmanned aerial vehicle based wireless sensor network for marine-coastal environment monitoring.," 2017.

[27] A. Malaver, N. Motta, P. Corke, and F. Gonzalez, "Development and Integration of a Solar Powered Unmanned Aerial Vehicle and a Wireless Sensor Network to Monitor Greenhouse Gases.," Sensors 2015, vol. 15, no. 2, pp. 4072-4096, 2015. DOI: 10.3390/s150204072.

[28] V. Klemas, "Coastal and environmental remote sensing from unmanned aerial vehicles: An overview.," Journal of Coastal Research, vol. 31, no. 5, pp. 1260-1267, 2015.

[29] Y. Zeng, R. Zhang, and T. J. Lim, "Wireless communications with unmanned aerial vehicles: opportunities and challenges," IEEE Communications Magazine, vol. 54, no. 5, pp. 36-42, 2016. DOI: 10.1109/MCOM . 2016.7470933.

[30] F. A. Administration. (2016). Summary of small unmanned aircraft rule (part 107), [Online]. Available: https://www.faa.gov/uas/media/Part_ 107_Summary.pdf (visited on 04/02/2019).

[31] DJI. (). Flame wheel arf kit, [Online]. Available: https://www.dji.com/ flame-wheel-arf/video.

[32] (2019). Pixhawk, [Online]. Available: http://pixhawk.org/.

[33] - (2019). Phantom 4 advanced, [Online]. Available: https://store. $\mathrm{dji}$. com/product/phantom-4-advanced? site=brandsite\&from=buy _ now_bar (visited on 04/02/2019).

[34] ArduPilot Dev Team. (). Installing mission planner (windows), [Online]. Available: http://ardupilot .org/planner/docs/mission-plannerinstallation.html.

[35] (). Accelerometer calibration in mission planner, [Online]. Available: http: //ardupilot.org/copter/docs/common-accelerometer-calibration. html.

[36] (). Compass calibration, [Online]. Available: http://ardupilot .org/ copter / docs / common-compass-calibration-in-mission-planner . html.

[37] (). Radio control calibration in mission planner, [Online]. Available: http: //ardupilot.org/copter/docs/common-radio-control-calibration. html.

[38] (). Electronic speed controller (esc) calibration, [Online]. Available: http: //ardupilot.org/copter/docs/esc-calibration.html.

[39] —, (2019). Radio failsafe, [Online]. Available: http://ardupilot.org/ copter/docs/radio-failsafe.html (visited on 04/02/2019).

[40] L. T. Nguyen. (2015). A new approach for collecting data in wireless sensor network, [Online]. Available: https : / / core . ac . uk / download / pdf / 61409584.pdf (visited on 04/02/2019). 
[41] Raspberry Pi Foundation. (). Installing operating system images on mac os, [Online]. Available: https://www.raspberrypi .org/documentation/ installation/installing-images/mac.md.

[42] R. Mitchell. (). Learn the differences between three common communication peripherals found on arduinos: Uart, spi, and i2c., [Online]. Available: https://maker .pro/arduino/tutorial/common-communicationperipherals-on-the-arduino-uart-i2c-and-spid.

[43] (). I2c, [Online]. Available: https://learn.sparkfun.com/tutorials/ i2c/all.

[44] (). Download the arduino ide, [Online]. Available: https : //www . arduino. cc/en/Main/Software.

[45] Atlas Scientific. (). Arduino i2c rtd sample code, [Online]. Available: https : //www.atlas-scientific.com/_files/code/rtd-i2c.pdf.

[46] Glassdoor. (2019). Environmental scientist salaries, [Online]. Available: https : / / www . glassdoor . com/Salaries / environmental-scientistsalary-SRCH_KO0, 23.htm (visited on 04/02/2019). 


\section{Appendices}

\subsection{Appendix A: Arduino I2C pH, Temperature, and Con- ductivity code}

Refer to Figures 71, 72, 73, 74, 75, and 76 for the Arduino code that controlled the operation of the temperature, $\mathrm{pH}$, and conductivity sensors using the $\mathrm{I}^{2} \mathrm{C}$ protocol.

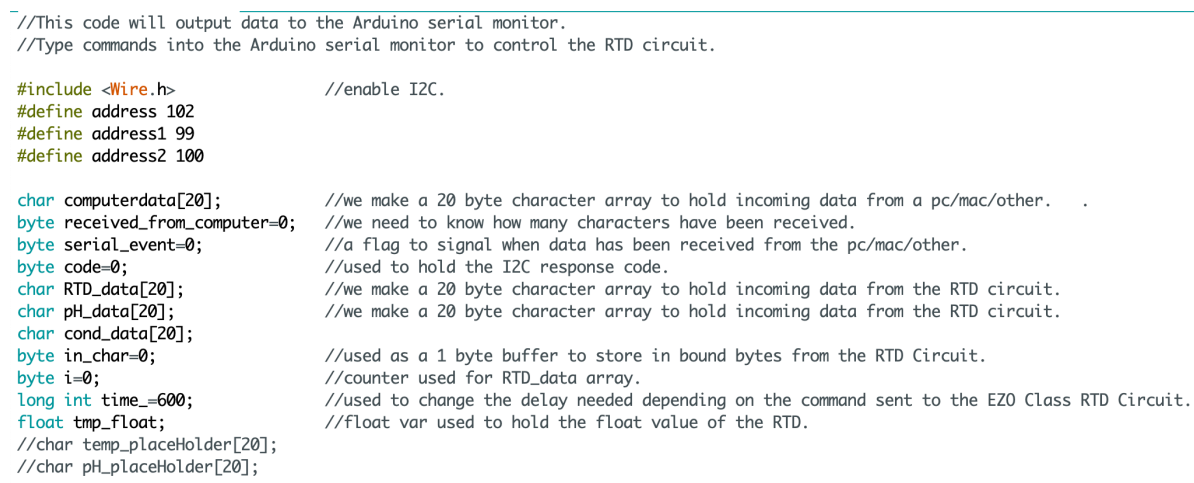

Figure 71: Arduino code controlling the three sensors.

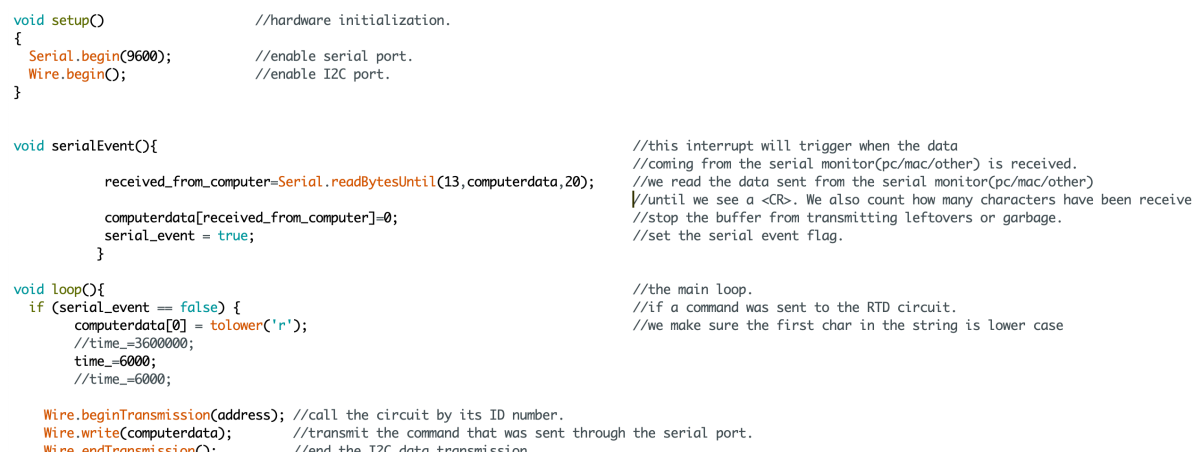

//if a cormand was sent to the RTD circuit.
computerdata $[0]=$ tolowere $\left(' r^{\prime}\right) ;$

//this interrupt will trigger when the dota //coming from the serial monitor(pc/mac/other) is received. V/until we see $a<\mathrm{CR}_{3}$. We also serial monitor(pc/mac/other) //stop the buffer from transmitting leftovers or garbage. / / we make sure the first char in the string is lower case

Figure 72: Arduino code controlling the three sensors. 


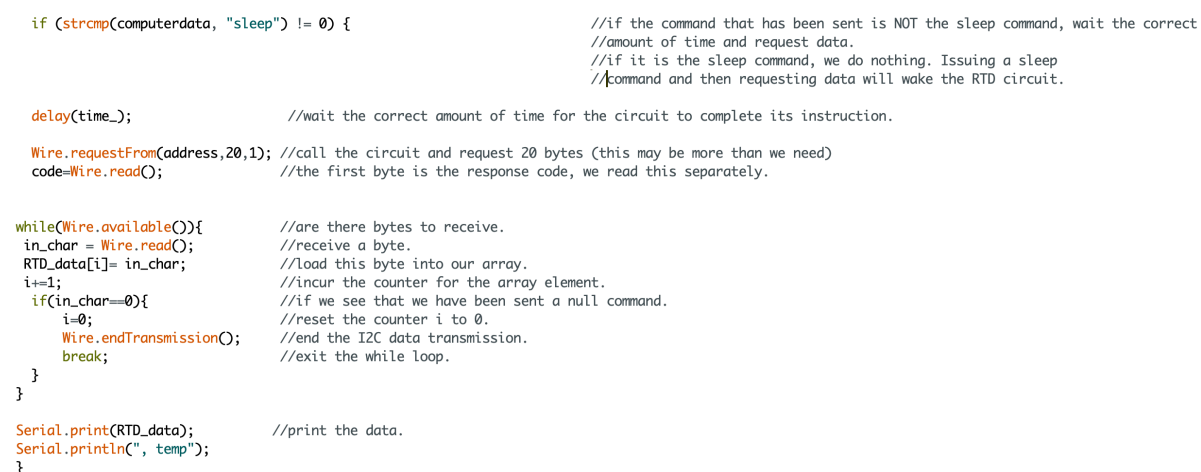

Figure 73: Arduino code controlling the three sensors.

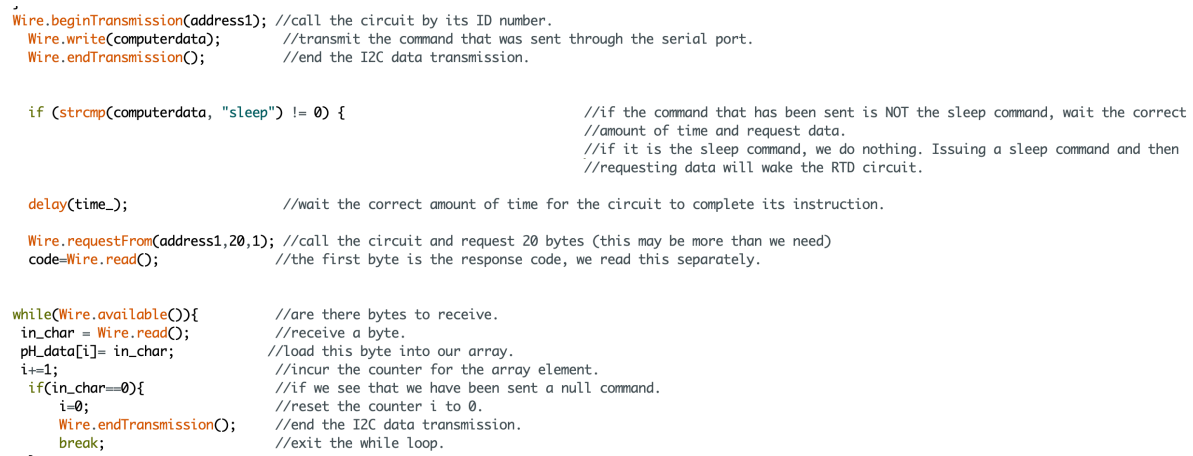

Figure 74: Arduino code controlling the three sensors.

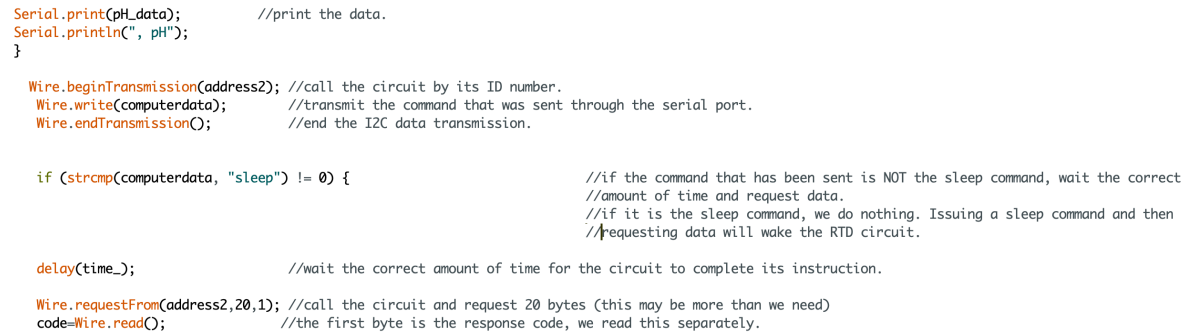

Figure 75: Arduino code controlling the three sensors. 


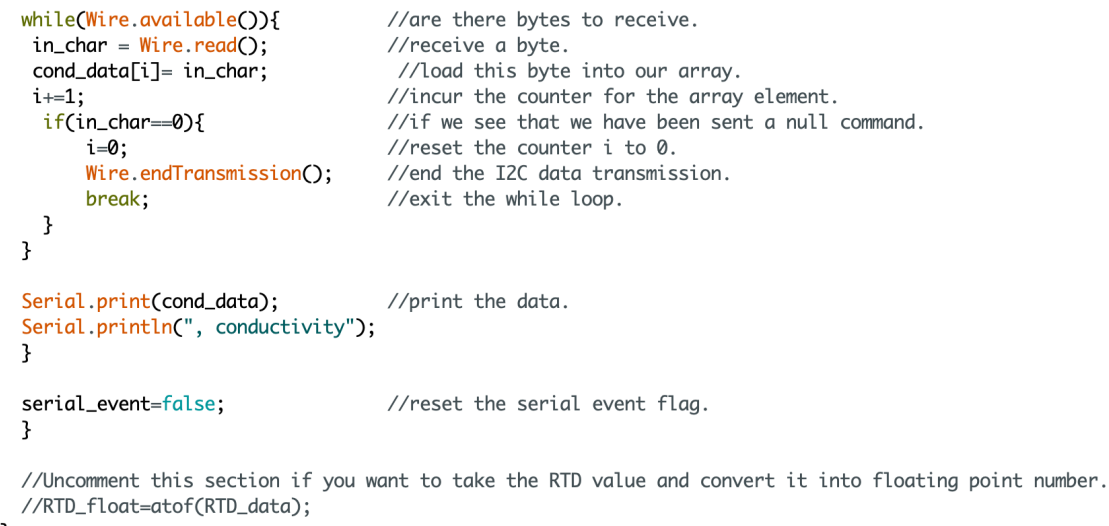

Figure 76: Arduino code controlling the three sensors.

\subsection{Appendix B: Arduino to Pi0 Python Code}

Refer to Figure 77 for the Python code that controlled the communication between the Arduino and Raspberry Pi 0.

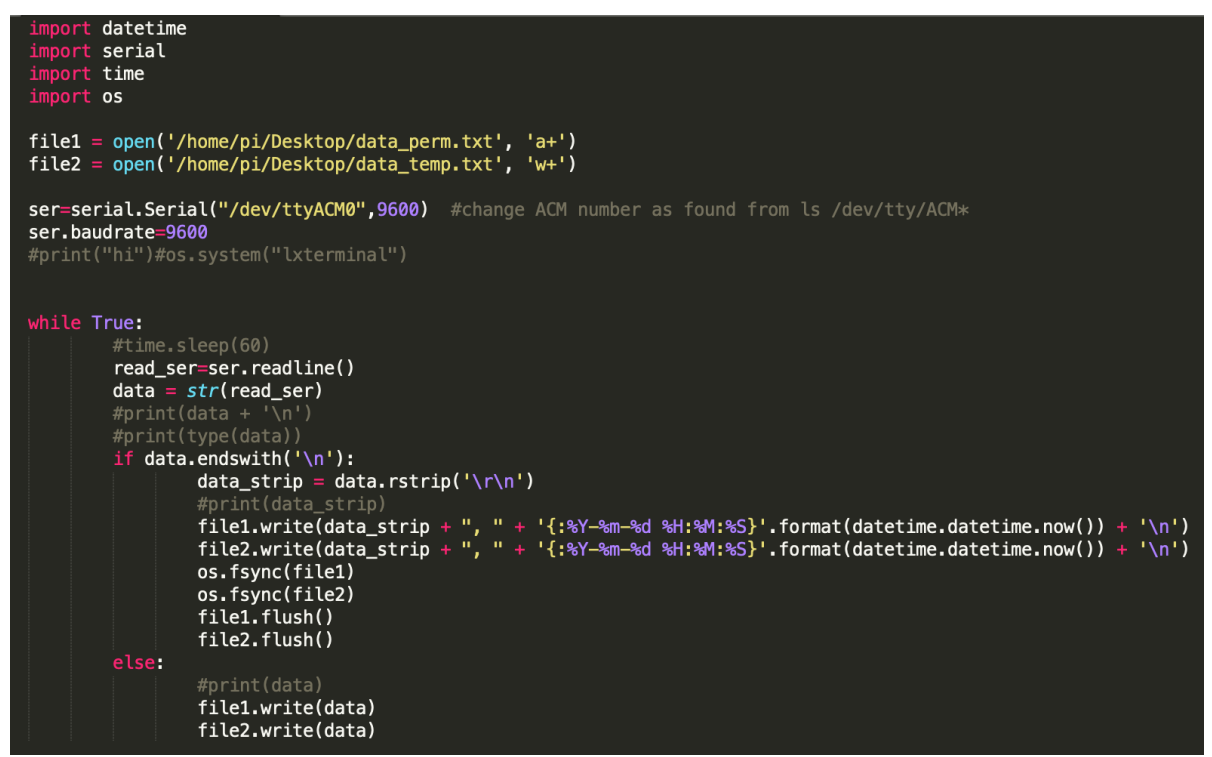

Figure 77: Python code controlling the communication between the Arduino and Raspberry Pi 0. 


\subsection{Appendix C: Python Code for Socket Coding Between $\mathrm{Pi} 0$ and $\mathrm{Pi} 3$}

Refer to Figure 78 for the client3 Python code that went on the Raspberry Pi 0 acting as the client.

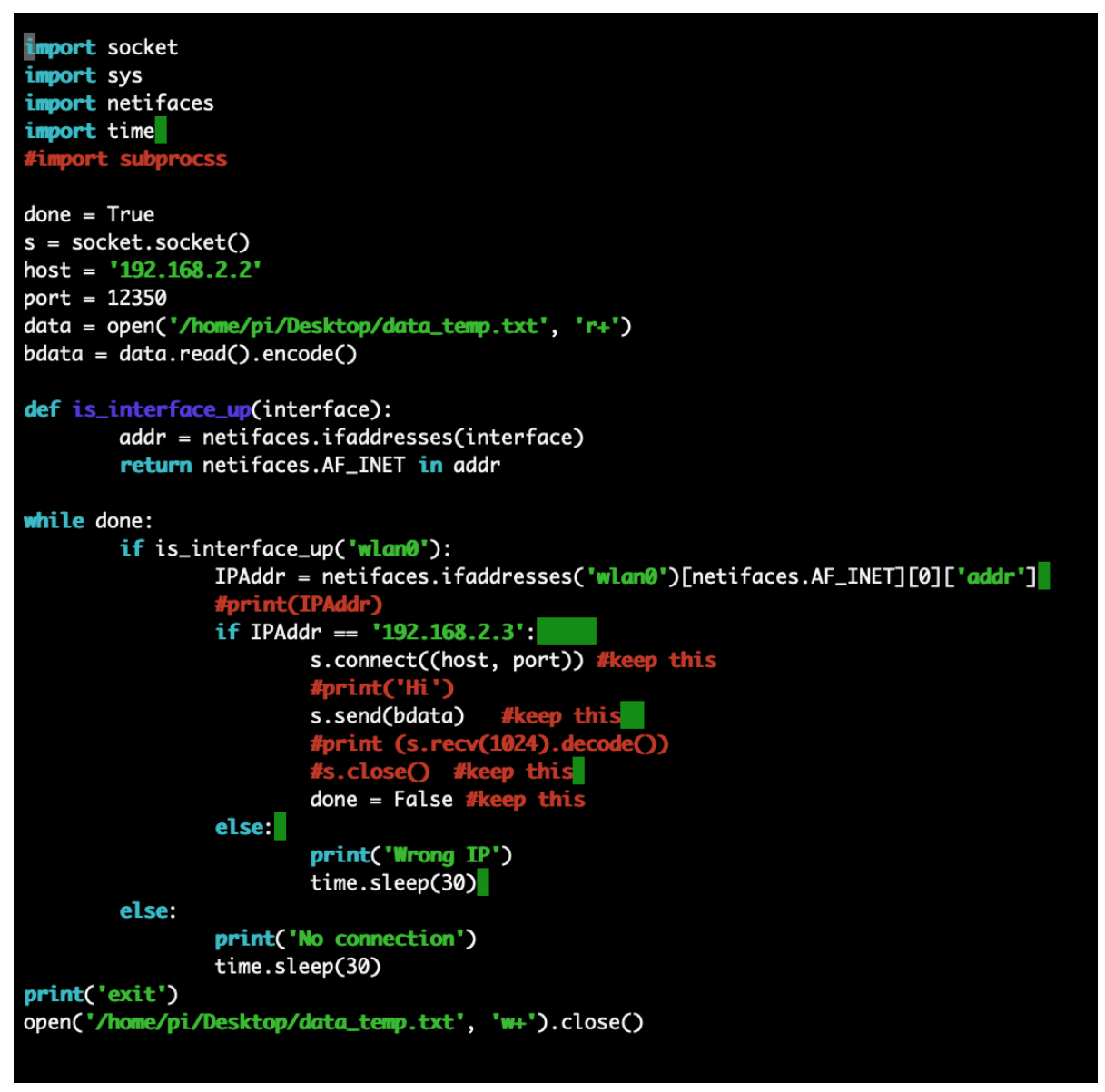

Figure 78: Python code that went in the client3.py file on the Raspberry Pi 0.

Refer to Figure 79 for the server Python code that went on the Raspberry Pi 3 acting as the server. 


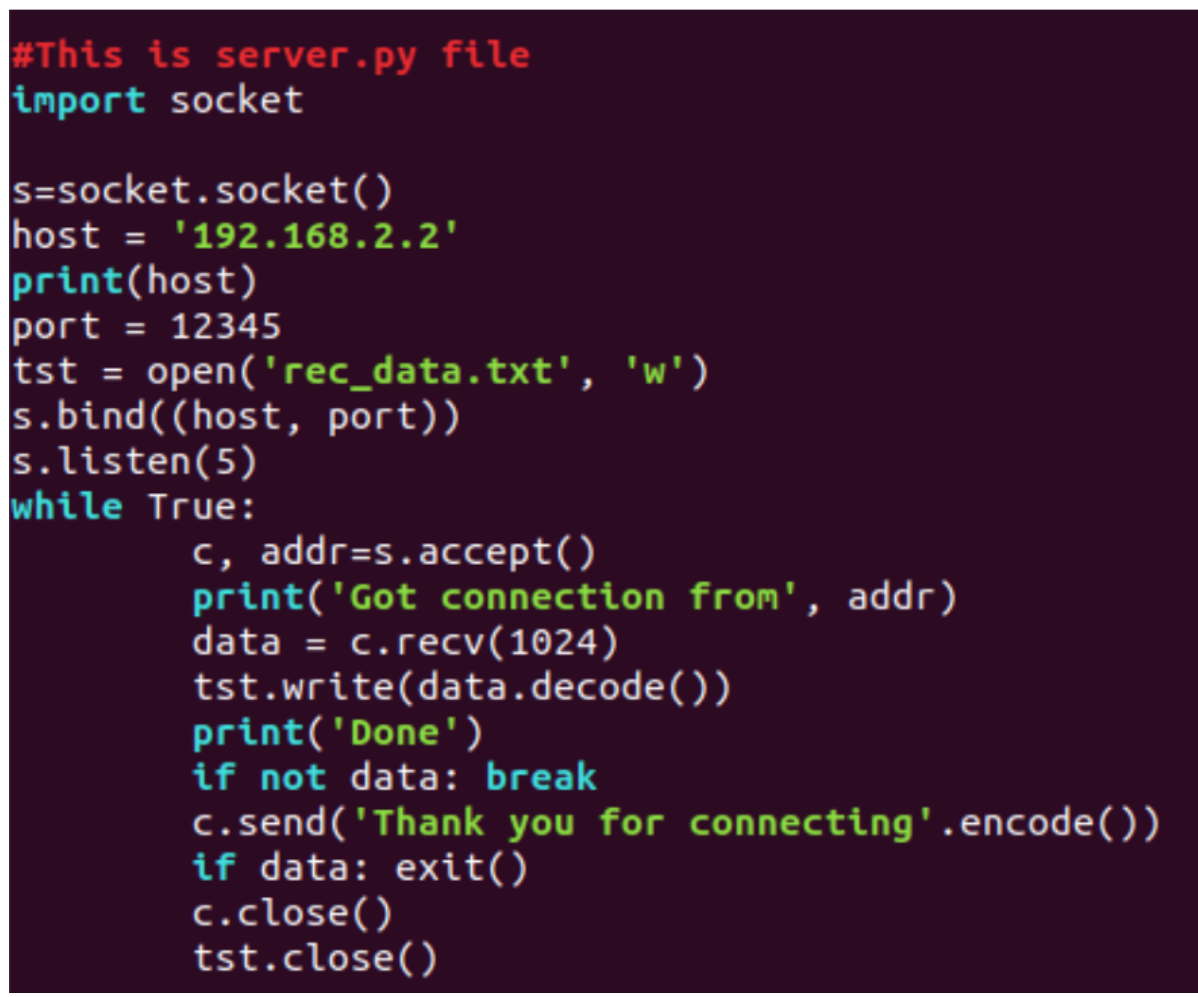

Figure 79: Python code that goes in the server.py file on the raspberry pi 3.

\subsection{Appendix D: Geographical Information System}

To fulfill the Honor's College requirement mandating that students submitting capstone projects through their respective programs of study must individually complete an additional component separate from non-Honor's College teammates, Laura Yates and Gina Valentino developed a geographic information system (GIS). The GIS was incorporated into a Story Map, a digital web mapping application available through Esri's ArcGIS Online software. The Story Map, entitled "Boones Run Story Map," is available at the following link: http://arcg.is/OyTPS8. The first page of the story map is shown in Figure 80. 


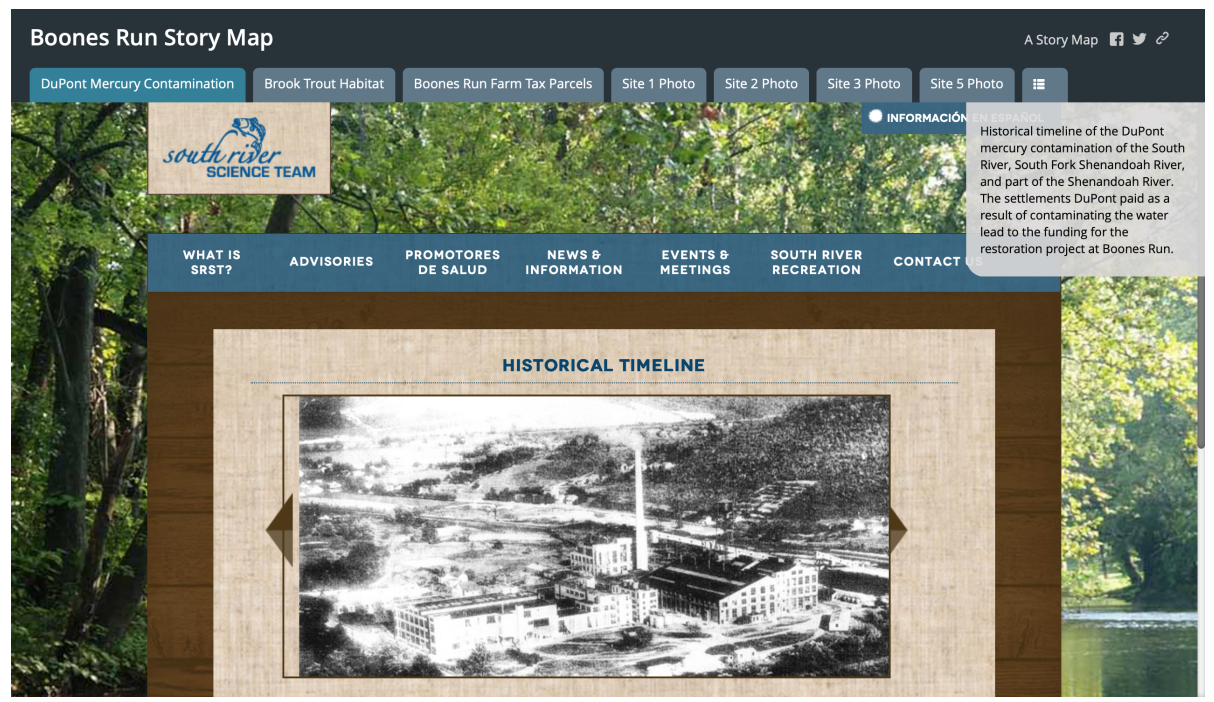

Figure 80: First screen of the story map created for this project.

The first tab on the Story Map, "DuPont Mercury Contamination," provides a historical timeline of the DuPont mercury contamination of the South River, South Fork Shenandoah River, and part of the Shenandoah River. The embedded website, developed by the South River Science Team, additionally provides visitors with a breadth of resources regarding the the South River.

The second tab ("Brook Trout Habitat") represents natural brook trout streams identified by the Virginia Department of Game and Inland Fisheries (VDGIF). Also included within this tab is a link to learn additional information about brook trout, an important indicator species for aquatic ecosystem health in the Shenandoah Valley and beyond. Note that the stretch of Boones Run between Sites $2-5$ is not identified as natural brook trout habitat by the VDGIF.

The third tab ("Boones Run Farm Tax Parcels") outlines Boones Run and identifies the four sampling locations selected for water quality monitoring. Furthermore, the tax parcels (designated in blue) illustrate the land the Virginia Department of Forestry will obtain to restore Boones Run. Water quality monitoring data, including $\mathrm{pH}$, water temperature, dissolved oxygen concentration and percent saturation, ambient air temperature, conductivity, total coliform units and total E. Coli concentration is available for perusal by selecting each individual site and clicking the backward and forward arrows to navigate to the desired date of data collection. Additional information included for each site includes latitude and longitude coordinates, a qualitative description, and notes for each date.

Tabs 4 - 7 provide a photograph of each site. 
The eighth tab, aptly named "Rockingham County Streams and Rivers," illustrates the vast network of streams and rivers within Rockingham County. Note the presence of the site markers as a frame of reference.

The ninth tab of the Story Map entitled "Boones Run Background Info" provides water quality data regarding Boones Run. Included within this embedded website is the United States Geological Survey's (USGS) unique identifier for Boones Run, the 8-digit Hydrologic Unit Code (HUC), the latitude and longitude of Boones Run, and the total drainage area of the watershed. Hyperlinks to the U.S. EPA's Surf Your Watershed database, USGS Science in Your Watershed site, National Water Census Data Portal, and USGS National Water Information System web page are directly accessible via this tab.

The tenth tab, "Rockingham County Tax Parcels," visually illustrates the land use associated with tax parcels in Rockingham County, bounded by the HUC12. Visitors to the Story Map can individually select each tax parcel to learn more information, including the address of each tax parcel, zoning codes, total acreage, and total property value. A drop-down legend on the top right corner of the page presents group classifications of tax parcels. Each tax parcel has a class code associated with it. Similar codes were grouped, such as agricultural land exceeding 100 acres and agricultural land below 100 acres. The codes were grouped for visual clarity.

The eleventh and final tab, entitled "Aerial Imagery versus Rockingham County Land Use," presents a tool that enables visitors to visually compare aerial imagery with tax parcels by clicking-and-dragging the "spy-glass." 